\title{
$\operatorname{xin} 2=+36$
}

Retersire t: 4 ayt to ba

tolki=1 If

NBS

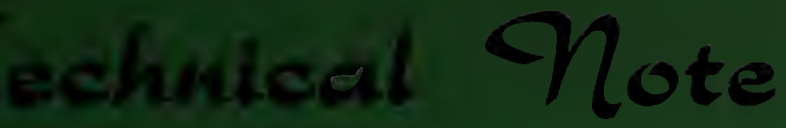

267

\section{PROCEDURES FOR PRECISE DETERMINATION OF THERMAL RADIATION PROPERTIES NOVEMBER 1963 TO OCTOBER 1964}

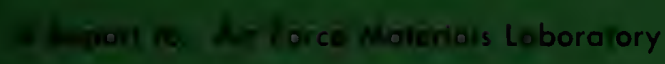

\author{
JOSEPH C. RICHMOND, S. THOMAS DUNN, \\ DAVID P. DEWITT, AND WARREN D. HAYES, JR.
}

Q. 5 DEPARTMENT OF COMMERCE WAFNOWAL BUREAU OF STANDARDS 


\section{THE NATIRNAL BLREAU OF STAND UBOS}

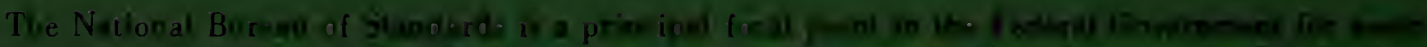

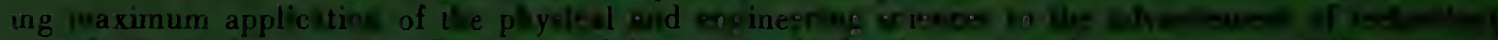

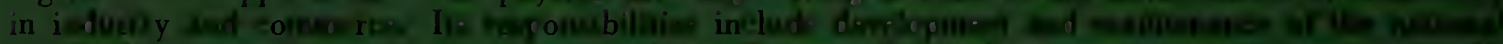

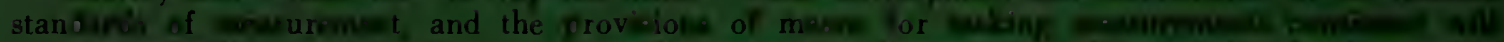

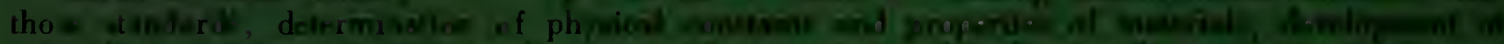

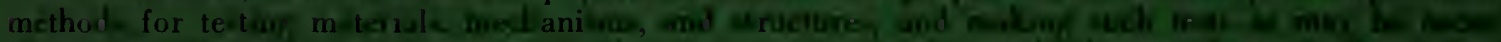

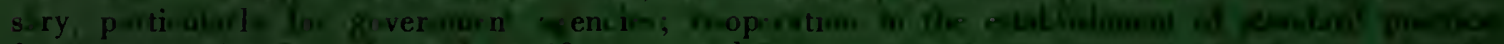

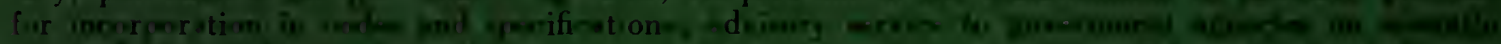

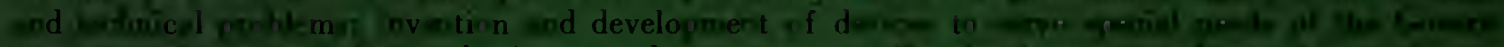

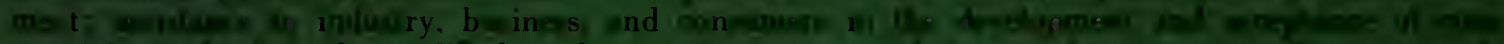

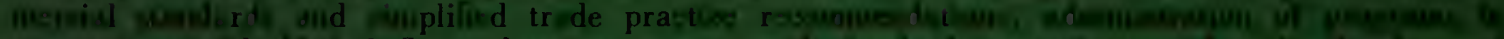

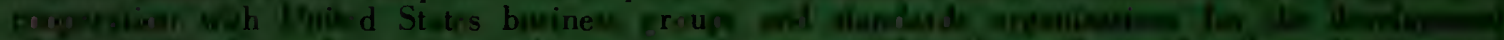

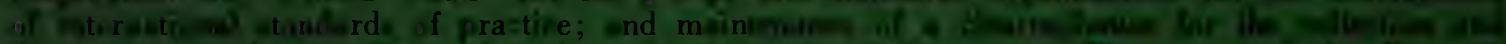

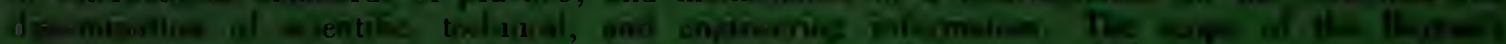

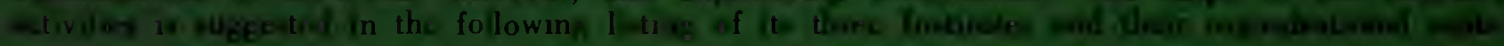

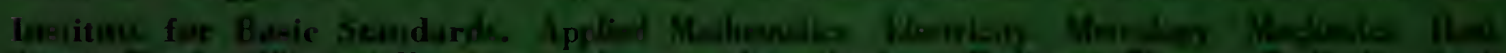

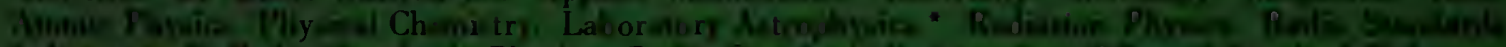

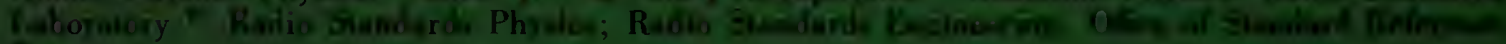
Data.

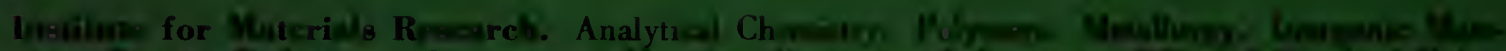

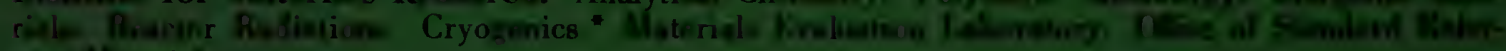
Fenon Metcrink

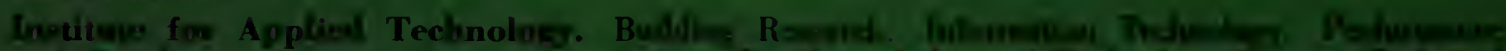

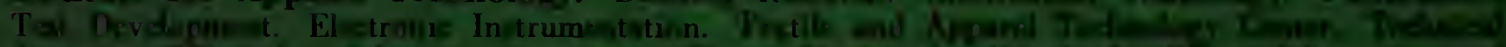

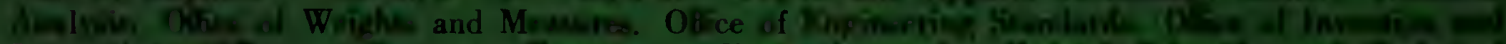

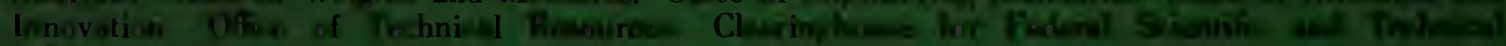
Iflomedion *

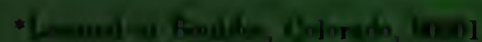

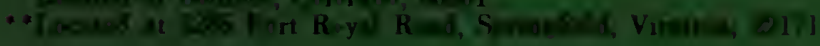




\title{
NATIONAL BUREAU OF STANDARDS Technical Note 267
}

ISSUED DECEMBER 17, 1965

\section{PROCEDURES FOR PRECISE DETERMINATION \\ OF THERMAL RADIATION PROPERTIES \\ NOVEMBER 1963 TO OCTOBER 1964}

\author{
Joseph C. Richmond, S. Thomas Dunn, \\ David P. DeWitt, and Warren D. Hayes, Jr.
}

A Report to

Air Force Materials Laboratory

Research and Technology Division

Air Force Systems Command

United States Air Force

Wright-Patterson Air Force Base, Ohio

Project No. 7381, Task No. 738103

(Prepared Under Contract No. DO (33-615)64-1005)

NBS Technical Notes are designed to supplement the Bureau's regular publications program. They provide a means for making available scientific data that are of transient or limited interest. Technical Notes may be listed or referred to in the open literature. 



\section{ABSTRACT}

A laser-source integrating sphere reflectometer was designed and built to measure the reflectance of specimens at high temperatures. The sphere was calibrated for linearity of response at $0.632_{\mu}$ by means of a shallow cylindrical cavity with a variable depth-to-radius ratio, having a lining of known reflectance. Preliminary tests showed that the flux emitted by a hot specimen at temperatures up to $2500^{\circ} \mathrm{K}$ will not invalidate the reflectance measurement. An ellipsoidal mirror reflectometer was calibrated for all known errors in the 1 to $7.5 \mu$ range. A preliminary analysis indicates that the errors in measurement of absolute reflectance with this equipment should not exceed 2 percent. A review of the literature on relation of thermal radiation properties to other properties of materials is presented, together with a summary of the work done in an effort to compute the spectral emittance of rhodium.

Key words: Emissivity, emittance, high temperature reflectance, infrared reflectance, radiation properties, reflectance, spectral emittance, spectral reflectance, thermal radiation. 


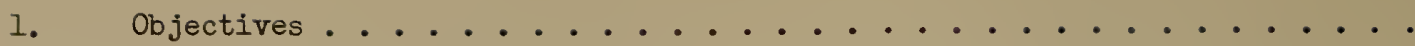

2. Laser-Source Reflectometer . . . . . . . . . . . . .

2.1 Background .

2.2 Theory of Integrating Spheres

2.3 Description of Instrument

2.4 Calibration of the Integrating Sphere Reflectometer . . . . . . .

2.5 Relative Reflectance Measurements ................

2.6 Modifications to Reflectometer .................

3. Ellipsoidal Mirror Reflectometer . . . . . . . . . . . . I 16

3.1 Background - Need for Instrument . . . . . . . . . . . . . 16

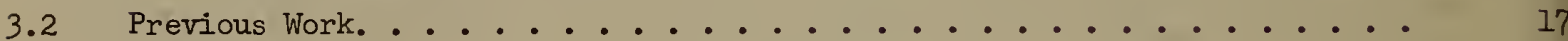

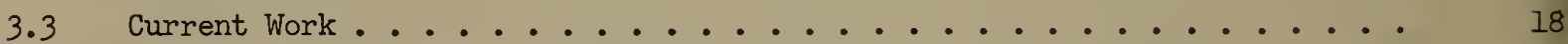

4. Summary of Work on Relaticn of Thermal Emittance to Other Properties . .

4.I Introduction .........................

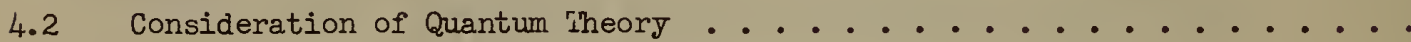

4.3 Electromagnetic Theory of Radiation ................

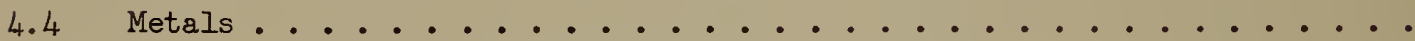

4.5 Optical Behavior of Rhodium ..................

4.6 Summary and Conclusions. . . . . . . . . . . . . .

5. Working Standards of Normal Spectral Emittance . . . . . . . . .

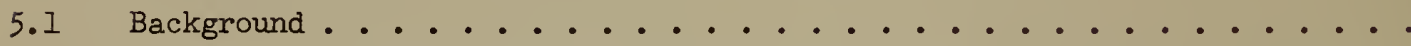

5.2 Comparison of Measured Normal Spectral Fmittance of Platinum to Values Predicted by the Hagen-Rubens Equation ................

5.3 Comparison of Standards Data with Literature Values ..........

6. Normal Spectral Emittance ....................

6.1 Background ..........................

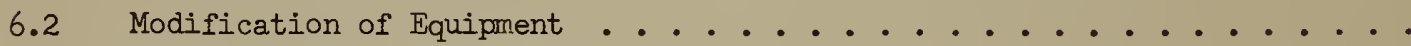

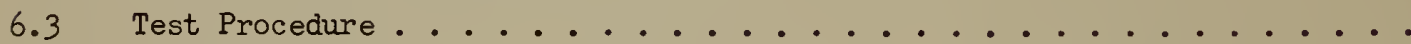

6.4 Measurements of Platinum ....................

7. Sumnary and Plans for Future Work ................. 
8. References ............................

9. Appendix A 
1. Schematic Diagram of High-Temperature Integrating Sphere Reflectometer ..

2. Cross Sectional View of Integrating Sphere ...............

3. Reflectance of $3 \mathrm{M}$ Velvet White Coating, Relative to MgO..........

4. Specimen Heater Designed to Heat $12.7 \mathrm{~mm}$ ( $1 / 2 \mathrm{inch}$ ) Diameter Specimens to Temperatures Up to About $800^{\circ} \mathrm{K}\left(1000^{\circ} \mathrm{F}\right) \ldots \ldots$

5. a. First Reflection Cavity

b. Lined Cavity ........................

6. Areas of Sphere Directly Illuminated by Flux Reflected from First

Reflection Cavity at Different L/R Ratios.

7. Schematic Diagram of Detector Assembly for Integrating Sphere

Reflectometers ...........................

8. Detector Configurations Used in First Reflection Cavity Tests

9. Difference Between Measured $F$ and Computed $f$, Plotted as a Function of $L / R$, for Detector Configurations with Black Sleeving, 10l-Black Sleeve with Light Pipe; 104-Black Sleeve Only; 107-Long Black Sleeve, 0.075 Inch Beam.

10. Difference Between Measured $F$ and Computed $f$, Plotted as a Function of $\mathrm{L} / \mathrm{R}$, for Detector Configurations with Collimators, 108 - $\frac{1}{2}$-inch Collimator, 0.075 Inch Beam; 109 - $\frac{1}{4}$-inch Collimator, 0.075 Inch Beam; 110 - Same as 109, Except EMI 5685 Photomultiplier Instead of IP28 . . . . . . .

11. Difference Between Measured $F$ and Computed $f$, Plotted as a Function of $L / R$, for Detector Configurations with White Sleeving, 105 - White Sleeve, Opal Glass View Cap and Light Pipe; 106 - Same as 105, but 0.075 Inch Beam; 111 - White Sleeve, Light Pipe, 0.075 Inch Beam.

12. Effect of Uncertainty in L/R on Accuracy of D Evaluated by Shallow Cavity Method. . . . . . . . . . . . . . . . . . . .

13. Effect of Uncertainty in Measured Reflectance Ratio, Qc/Qw, on Accuracy of $\rho$ Evaluated by Shallow Cavity Method. . . . . . . . . . . . . .

14. Absolute Reflectance of 3M Velvet Yellow Paper, Computed from Data of Trials 101 and 102, Top, and $103^{\circ}$ and 104, Bottom, Plotted as Functions

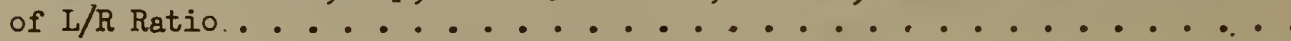

15. Absolute Reflectance of 3M Velvet Yellow Paper, Computed from Data of Trials 111 and 112, Top, and of 3M Velvet Green, Computed from Data of Trials 301 and 302, Bottom, Plotted as Functions of L/R Ratio. . . . . .

16. Experimentally Determined F Values and Absolute Reflectance of of $3 \mathrm{M}$ Velvet Yellow Paper, Computed by Use of Equation (7), Trials 101-102 . . .

17. Experimentally Determined F Values and Absolute Reflectance, Rw, of 3M Velvet Yellow Paper, Computed by Use of Equation (7), Trials 103104. 
18. Experimentally Determined F Values and Absolute Reflectance, P, of $3 \mathrm{M}$ Velvet Yellow Paper, Computed by Use of Equation (7), Trials 111-112. . .

19. Experimenially Determined F Values and Absolute Reflectance of $3 \mathrm{M}$ Velvet Green Paper, Computed by Use of Equation (7), Trials 301-302 . . .

20. Ratio of First Reflected Flux to Total Flux, $\mathrm{K}$, Reflected by a Shallow Cavity for Different L/R Ratios, as a function of Wall Reflectance, ${ }^{\circ} W^{*}$.

21. Relation of Reflectance, $p$, to Measured Reflectance Ratio, $Q_{S} / Q_{F}$, in the Appended Sphere Method, for Different Sphere Geometries, d/D. . . . .

22. Effect of Change in Measured Reflectance Ratio, QS/QF, on Reflectance by the Appended Sphere Method, Plotted as a Function of Reflectance, $\rho$, for Different Sphere Geometries, d/D................

23. Effect of Change in Sphere Geometry, $d / D$, on Reflectance by the Appended Sphere Method, Plotted as a Function of Reflectance, $p$, for

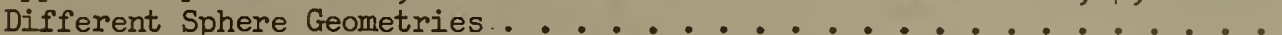

Spatial Sensitivity of the Golay-Cell Detector in the A-A' Direction. . .

Spatial Sensitivity of the Golay-Cell Detector in the B-B' Direction. .

Spatial Sensitivity of the Thermopile Detector in the $A-A^{\prime}$ and $B-B^{\prime}$

Directions to Chopped Incident Flux ...............

27. Spatial Sensitivity of the Thermopile Detector in the $A-A^{\prime}$ and $B-B^{\prime}$ Directions to Unchopped Incident Flux.

28. Angular Sensitivity of the Thermopile Detector in the $A-A^{\prime}$ Plane at

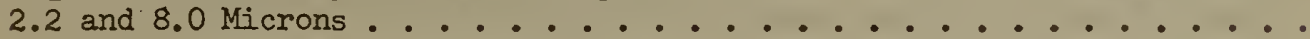

29. Angular Sensitivity of the Thermopile Detector in the B-B' Plane at 2.2 and 8.0 Microns ...................

30. Diffusing Elbow Designed to Reduce Spatial Sensitivity of Thermopile

Detector. ...........................

31. Angular Sensitivity of Thermopile Detector with Diffusing Elbow, in the Plane Perpendicular to the Diffusing Surfaces . . . . . . . . . .

32. Angular Sensitivity of the Thermopile Detector with Diffusing Elbow, in the Plane Parallel to the Diffusing Surfaces. ............

Diffusing Elbow with Mirror Walls Except for $45^{\circ}$ Diffusely Reflecting

Plane Surfaces. ....................

Goniophotometric Reflectance Curve of Machined Surface Consisting of Spherical Depressions in Aluminum, for $45^{\circ}$ Angle of Incidence......

Schematic Diagram of Optical System Used in Evaluation of Coatings for Diffusing Spheres .....................

Angular Sensitivity of Thermopile Detector with 4-inch Diameter MgO Coated Sphere, at 1.5 and 3.0 Microns . . . . . . . . . . . . 
38. Angular Sensitivity of Thermopile Detector with 2-inch Diameter L-Shot Gold-Plated Sphere at 2.2 Microns. . . . . . . . . . . . . .

39. Angular Sensitivity of Thermopile Detector with 2-inch Diameter S-460 Shot Gold-Plated Sphere at 2.2, 5 and 8 Microns ........

40. Angular Sensitivity of Thermopile Detector with 2-inch Diameter Sphere Coated with Hand-Peened mu Sulfur, at 1.5, 2.2, 5.0 and

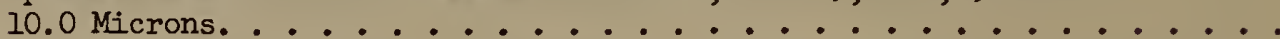

41. Response Ratio of Thermopile Detector with Indicated Spheres, Plotted as a Function of the Ratio of the Illuminated Areas on the Sphere Wall, for Beams of Constant Flux. . . . . . . . . . . . . .

42. Detector and Beam Configurations for a Test of Efficiency of a

Material as an Integrating Sphere Coating . . . . . . . . . . . .

43. Theoretical Model for Developing Theory of Tests of Integrating Sphere Coatings. . . . . . . . . . . . . . . . . . . . . .

44. Test Configurations Used to Obtain Data for Calibration of Reflectometer.

45. Projection of Hole, Sample and Specularly Reflected Image in First Focal Plane, Top, and Shield B, Bottom. ...............

46. (a) Experimental Values of $n$ and $k$ for Mercury $[45,56,47,48,49]$ Compared to Values Computed by the Drude [16] Theory.

(b) Experimental Values of Reflectivity of Mercury-Glass and MercuryQuartz Interfaces (Points) Compared to that Computed from the Drude Theory (Curves).

(c) Experimental Values of Reflectivity of Mercury - NaCl Interface (Points) Compared to that Computed from the Drude Theory (Curve).

47. Comparison of the Reflectivity of Rhodium with Values Predicted by the Multi-Free/Bound Electron Model . . . . . . . . . . . . .

48. The Effect of Individual Terms of the Multi-Free/Bound Electron Model on Emissivity.....................

49. Normal Spectral Emittance of Platinum Working Standards at $800^{\circ} \mathrm{K}$, Compared to Values Computed from the Hagen-Rubens Equation. . . . . . .

50. Normal Spectral Emittance of Platinum Working Standards at $1100^{\circ} \mathrm{K}$, Compared to Values Computed from the Hagen-Rubens Equation.

51. Normal Spectral Emittance of Platinum Working Standards at $1400 \circ \mathrm{K}$, Compared to Values Computed from the Hagen-Rubens Equation. . . . . . .

52. Normal Spectral Emittance of Platinum-13\% Rhodium Alloy at $800 \circ \mathbb{K}$. . .

53. Normal Spectral Emittance of Platinum-13\% Rhodium Alloy at $1100^{\circ} \mathrm{K}$, Compared to Values Computed from the Hagen-Rubens Equation. . . . . . .

54. Normal Spectral Fmittance of Platinum-13\% Rhodium Alloy at $1300^{\circ} \mathrm{K}$, Compared to Values Computed from the Hagen-Rubens Equation. . . . . . .

55. Photomicrograph of Platinum-13\% Rhodium Working Standard, Unetched, $\times 250$.. 
56. Electron Backscattering Image of Platinum-13\% Rhodium Working

Standard, $x 440$. . . . . . . . . . . . . . . . .

57. Silicon Ka X-ray Image of Platinum-13\% Rhodium Working Standard,

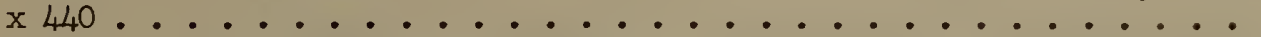

58. Normal Spectral Emittance of Platinum Working Standards at $1400 \circ \mathrm{K}$

Compared to Literature Values

59. Normal Spectral Fmittance of Oxidized Inconel Working Standards at $800^{\circ} \mathrm{K}$ Compared to Literature Values.

60. Normal Spectral Fmittance of Oxidized Inconel Working Standards at $1100^{\circ} \mathrm{K}$, Compared to Literature Values. . . . . . . . . . . .

61. Normal Spectral Emittance of Oxidized Inconel Working Standards at $1300^{\circ} \mathrm{K}$, Compared to Literature Values. . . . . . . . . . . .

62. Normal Spectral Emittance of Platinum Working Standard, Measured with

Sodium Chloride Prism Mounted in Spectrometer, Circles, and Cesium Bromide Prism Mounted in Spectrometer, Triangles. . . . . . . . . . . 
1. Data Obtained with $3 \mathrm{M}$ Velvet yellow coating on paper in First Reflection

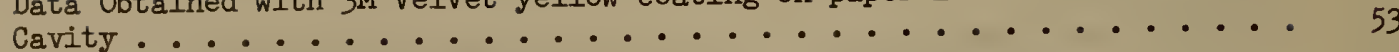

2. Data Obtained with Shallow Cavity Iined with 3M Velvet Paper . . . . 54

3. Absolute Reflectance Computed from Shallow Cavity Measurements . . . . 54

4. Reflectance of Various Materials at 0.6328 Micron, Relative to $3 \mathrm{M}$ Velvet

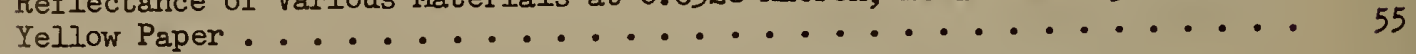

5. Parameters and Relations Descriptive of Wave-Matter Interaction. . . • 56

6. Typical Values of Parameters Descriptive of Wave-Matter Interaction. . • 56

7. Classical Models for the Prediction of the Emissivity of Metals. . . • . 57

8. Theoretical Models for Prediction of the Optical Properties of Metals . 58

9. Comparison of the Roberts' and Multi-Free/Bound Electron Model Constants • 58

10. Parameters for the Roberts' Model for Various Metallic Conductors. . . . 59

11. Multi-Free/Bound Electron Model Parameters for Rhodium . . . . . . 60

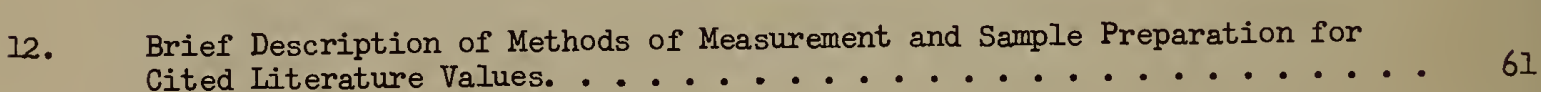

13. Table of Average Standard Deviations . . . . . . . . . . . 62

14. Table of Average Measurements. . . . . . . . . . . . . 62 


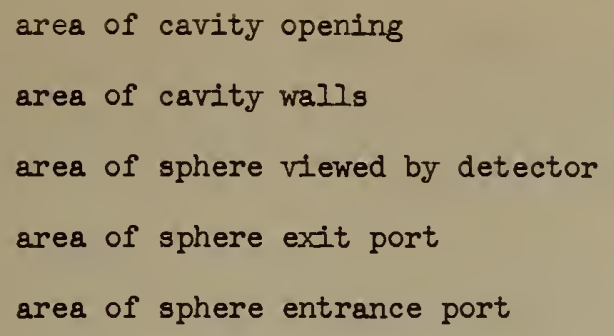

In $\mathrm{V}$

measured reflectance of first reflection cavity

type of free electrons $-g=1,2,3 \ldots \ldots$

type of bound electrons $-h=1,2,3 \ldots \ldots$

$P_{c} / P_{W}$, ratio of cavity reflectance to wall reflectance 


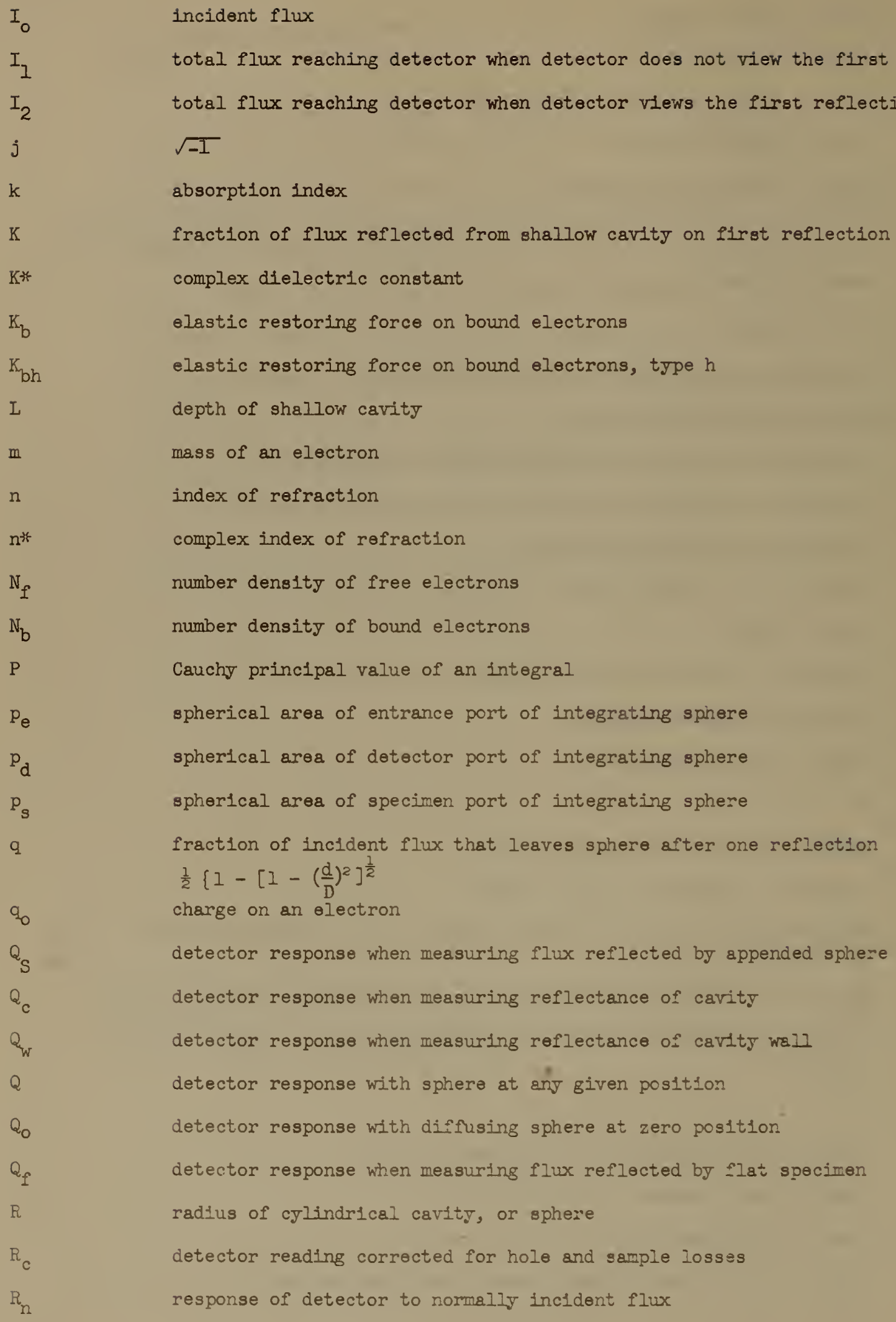


flux reflected by sphere wall on first reflection

response of detector to flux incident at angle $\theta$

detector reading for normally incident flux

detector reading for reflected flux with no shield

detector reading for reflected flux with shield B in plice

$R_{1}-R_{2}$, measure of the flux striking shield $B$

flux reflected twlce by sphere wall

flux reflected twice by sphere wall that reaches detector

flux reflected three times by sphere wall that reaches detector

flux reflected $\mathrm{n}$ times by sphere wall that reaches detector

flux reflected by sphere wall on first reflection that reaches detector

total area of sphere wall

$S-p_{e}-p_{d}-p_{g}$, total reflecting area of sphere wall

time

real component of complex quantity $V$

$v \exp j \theta, v^{2}=p(0, \lambda)$

distance traveled in time $t$

displacement of electron

velocity of electron

acceleration of electron

impedance ratio

intrinsic impedance

intrinsic impedance of free space

attenuation factor

phase factor

$\alpha+j \beta$

viscous damping force for free electrons

permittivity of free space

emissivity for flux polarized normal to the plane of measurement

emissivity for flux polarized parallel to the plane of measurement

normal spectral emissivity at wavelength $\lambda$ 
$\varepsilon(\varphi, \lambda)$

$\theta$

$\lambda$

$\lambda_{0}$

$\lambda_{f}$

$\lambda_{f g}$

$\lambda_{\text {bh }}$

$\lambda_{\text {oh }}$

$\mu$

$\mu^{* *}$

$\pi$

$\rho$

$\rho_{\mathrm{C}}$

$\rho_{\mathrm{m}}$

$\rho_{\mathrm{S}}$

$\rho_{S}$

$\rho(0, \lambda)$

$\rho(\varphi, \lambda)$

$\rho_{\text {st }}$

$\rho_{W}$

$\rho(\psi, \varphi, \lambda)_{n}$

$\rho(\psi, \varphi, \lambda) p$

$T$

$\varphi$

$\psi$

$\omega$ spectral emissivity in direction $\varphi$ at wavelength $\lambda$

phase component of complex quantity, $\mathrm{V}$

wavelength, or wavelength of incident wave

c/qo $\left(\frac{\mathrm{Tm}}{\mathrm{N}_{\mathrm{f}}}\right)^{\frac{1}{2}}$

$2 \pi c \tau$, Viscous damping parameter for free electrons

viscous damping parameter for free electrons, type $g$

viscous damping parameter for bound electrons, type $h$

resonant wavelength of bound electrons, type $h$, with no damping forces present

microns

magnetic permeability

$3.14159 \ldots$

reflectance

reflectance of a cavity

resistivity of a metal

reflectance of specimen

reflectance of sphere wall

specular reflectance for normal incidence, at wavelengtin ,

specular spectral reflectance for radiation licident at angle $\varphi$

reflectance of standard

reflectance of cavity wall

reflected component polarized normal to the plane of incidence

reflected component polarized parallel to the plane of Incidence

relaxation time

angle of refraction, measured from the norms

angle of incidence, messured from the normsi

Prequency 
The over-a.1] objective of this project was to develop equipment and procedures for obtaining reliable measurements of the thermal radiation properties of materials, and to select, prepare, and calibrate physical standards for use by other laboratories in checking their equipment and procedures. The specific temperature range of interest was 800 to 2500 oK (about 1000 to 4000 \%). Procedures applicable to both metallic and non-metallic (ceramic) naterials heated in air, controlled atmosphere and vacuum were desired.

The specific objectives for the report period included the following. (1) A study of the feasibility of evaluating normal spectral emittance at high temperature by measurement of reflectance by means of a laser-source integrating sphere reflectometer. Reflectance will be measured at a few specific wavelengths, and values at intermediate wavelengths will be interpolated from the continuous spectral reflectance curve of the material measured at room temperature with a different instrument. (2) Procurement, preparation, and calibration of standards of normal spectral emittance in the temperature range of 1400 to 1900 \% (approximately 2000 to $3000 \mathrm{~F}$ ). (3) Extension of the wavelength range of the normal spectral emittance equipment in the $800^{\circ}$ to $1400^{\circ} \mathrm{K}$ (approximately 1000 to $2000^{\circ} \mathrm{F}$ ) temperature range to wavelengths of 15 to 35 microns.

Development of equipment and procedures for measurement of normal spectral emittance by the rotating cylinder method and of total normal emittance by the shallow cavity method has proceeded on another project sponsored by the George C. Marshall Space Flight Center of NASA. Reports covering work on both projects are sent to both sponsors.

\section{Leser-Source Reflectometer}

\subsection{Background}

Spectral emittance measurements obtained by direct comparison of the spectral radiant flux density of a hot specimen to that of a laboratory blackbody furnace at the same temperature are subject to large errors, not only at wavelengths that are appreciably shorter than that at the peak of the Planck distribution function at the test temperature, (see Figure 1 , Reference 56), but also at temperatures above those at which standard thermocouples can be used.

The errors at the short wavelengths arise from two sources: (1) The energy available for measurement decreases rapidly with decreasing wavelength at wavelengths below the peak; hence, there is not enough energy to permit measurements of the highest accuracy at the shortest wavelengths. (2) The temperature dependence of spectral flux density increases rapidly with decreasing wavelength at wavelengths below the peak; therefore, a small temperature difference between specimen and blackbody reference will cause a much larger error in emittance at wavelengths shorter than the peak than at wavelengths longer than the peak.

At temperatures above those at which standard thermocouples can be used, the temperature scale is based upon the optical pyrometer, and either an optical pyrometer or a radiation pyrometer is commonly used for temperature measurements in this range. These instruments are accurate for measuring the temperature of a laboratory blackbody furnace, and they also measure the brightness temperature or the radiance temperature of a specimen with acceptable accuracy. Conversion of this brightness or radiance temperature to true temperature, however, requires knowledge of the spectral emittance of the specimen over the appropriate wavelength range. Thus the emittance must be known in order to measure the temperature, which in turn must be known to evaluate the emittance.

Reflected flux, as contrasted to emitted flux, is nearly independent of the temperature of the specimen, at least over temperature intervals that do not exceed about $50^{\circ} \mathrm{K}$. For opaque specimens, the emittance is equal to one minus the reflectance, by Kirchhoff's law. The measurement of reflectance involves only the comparison of incident and reflected flux. Thus, such measurements would appear to offer the possibility of considerably increasing the precision with which emittance can be determined, particularly in the short-wavelength and high-tempera-

The nomenclature used in this report is that used in references 51 through 56, and is defined in detail in references 51 and 54. 
ture regions where direct measurements are subject to large errors. However, for reflectance to be equal to one minus the normal spectral emittance, the reflectance must be measured under conditions of either normal illumination and hemispherical viewing, or diffuse illumination and normal viewing.

Three general methods have been used to measure reflectance under these conditions; by using (1) integrating sphere, (2) integrating hemisphere, and (3) hemispherical source instruments. Integrating sphere instruments are generally preferred, and are the type most widely used at wavelengths for which suitable sphere coatings are available. The principal problem in measuring reflectance of a specimen at high temperatures is to distinguish between the flux reflected by the specimen and that emitted by the hot specimen. This can be done by chopping the beam of incident flux, so that an a-c signal is produced by the detector, which is synchronously amplified and recorded. The $d-c$ signal from the flux emitted by the hot specimen is not amplified, and appears only as noise. The signal-to-noise ratio can be increased by using a source of high flux density within the wavelength interval of interest. helium-neon continuous wave laser was selected for this purpose, because its flux density within the narror wavelength band at which it lases is several orders of magnitude larger than the flux emitted by any solid specimen at a temperature below its melting point, within the same wavelength interval.

\subsection{Theory of Integrating Spheres}

The ideal integrating sphere for use in reflectance measurements consists of a hollow sphere with three small openings in the wall. The inside wall has a completely diffusing surface of high reflectance. The specimen is placed over one of the openings, called the specimen port, in a position such that it forms a portion of the sphere wall. It is illuminated by a beam of light projected onto its surface through a second opening, called the entrance port. A detector views a portion of the sphere wall through the third opening, called the detector port. The flux initially reflected by the specimen is multiply reflected by the sphere wall and uniformly distributed over its surface. If the detector and the area of the sphere wall that it views are shaded from the specimen, the response of the detector will be proportional to the reflectance of the specimen, regardless of the geometric distribution of the reflected flux (assuming that a negligible fraction is lost out the openings).

When a standard of known reflectance is substituted for the specimen, the response of the detector is likewise proportional to the reflectance of the standard, and the reflectance of the specimen can be computed from the known reflectance of the standard and the measured ratio. This is usually referred to as operation in the substitution mode.

Unfortunately, all integrating spheres depart from the ideal conditions to some extent, and corrections must be made for the multiply-reflected flux lost out of the openings, and that absorbed by the specimen. Such corrections can be kept small by keeping the area of the openings small compared to the total inside area of the sphere. Also, no real sphere coating is completely diffusing, and the reflectance of the sphere wall may vary over its surface. However, in spite of rather large departures from ideal conditions, carefully constructed integrating sphere reflectometers do give useful reflectance data.

The theory of the integrating sphere reflectometer for a perfectly diffuse sphere wall and diffusely reflecting specimens has been presented by Jacquez and Kuppenheim [1] 1 . For measurements by the substitution method (in which first the standard and then the specimen is placed over the specimen port and measured) the reflectance of the specimen, $\rho_{\mathbf{g}}$, is expressed by

$$
\frac{B_{s}}{B_{s t}}=\frac{\rho_{s}}{\rho_{s t}} \quad\left[1-\frac{\left(\rho_{s t}-\rho_{s}\right)_{P_{s} / S}}{1-\rho s / S-\rho_{s} p_{s} / S}\right]=C \frac{\rho_{s}}{s t}
$$

or

$$
\rho_{s}=\frac{\rho_{s t}{ }^{B} s}{C B_{s t}}
$$

\footnotetext{
IFigures in brackets refer to papers cited in Section 8 Feferences.
} 
$B_{S}=$ brightness at detector port with specimen at specimen port.

$B_{\text {st }}=$ brightness at detector port with standard at specimen port.

$\rho=$ reflectance of sphere wall.

$p_{e}=$ spnerıcal area of entrance port.

$\mathrm{p}_{\mathrm{d}}=$ spherical area of detector port.

$\mathrm{p}_{\mathrm{S}}=$ effective spherical area of specimen port.

$S=$ total area of sphere $\operatorname{wall} 4 \pi R^{2}$

$S^{\prime}=S-p_{e}-p_{d}-p_{S}$ total reflecting area of sphere wall.

$C=$ calibration factor for sphere, $\left[1-\frac{\left(p_{s t}-p_{S}\right) p_{s} / S}{1-\rho S^{1 / S}-p_{s} p_{s} / S}\right]$.

The equation for $\mathrm{C}$ reduces to

$$
c=\frac{1-\rho S^{1 / S}-\rho_{S t} P_{S} / S}{1-\rho S^{1 / S}-\rho_{S} p_{S} / S}
$$

The ratio $\mathrm{B}_{\mathrm{S}} / \mathrm{B}_{\mathrm{st}}$ is the ratio of the reflectometer readings for the specimen and standard, respectiviely, and $C$ is the correction factor for flux losses through the sphere ports.

The theory given above is for the perfect sphere, where the specimen and standard have a spherical surface of the same radius as the sphere, and are diffuse. It can be modified for the case of flat specimens. No theory is available for specularly reflecting specimens.

\subsection{Description of Instrument}

The integrating sphere reflectometer that was designed for the present work as described in reference 55 is shown schematically in Figure 1. It consists of a source, the integrating sphere proper, and a detector, plus auxiliary equipment.

A helium-neon continuous-wave gas laser is used as the source. It produces a slightly diverging beam of plane-polarized coherent radiation about $2 \mathrm{~mm}(0.08 \mathrm{in.})$ in diameter at the source. The laser will generate narrow-band radiation at three different wavelengths, $0.6328,1.15$, and 3.39 microns. Because the dielectric-coated mirrors reflect the desired wavelength and absorb the others, the lasing action is confined to the desired single wavelength. Total power output is about one milliwatt. When the power input is properly adjusted, the laser output in a dust-free atmosphere is stable to better than 0.5 percent for periods of several hours. Input power, however, is critical. When the power is not properly adjusted, the laser output varies sinusoidally with an amplitude variation of 2 to 5 percent and a period that varies from about 10 minutes to an hour or more, depending on the power setting.

The Brewster-angle windows on the gas-discharge tube collect a high static charge, so that dust paritcles in the atmosphere are attracted to them. Such dust particles on the windows seriously reduce the power output of the laser. In order to obtain the desired stability, it was necessary to operate the laser in a dust-tight enclosure.

The laser beam passes out of the enclosure through a window, after which it is chopped at 13 cps. The chopped beam is then directed downward through the entrance port of the sphere to strike the specimen at an angle of about $6^{\circ}$ to the normal. The beam is about $7.5 \mathrm{~mm}$ in diameter ( $0.3 \mathrm{in.})$ at the specimen, but can be reduced to a diameter of about $2 \mathrm{~mm}$ ( 0.075 in.) by means of a long focal length lens inserted in the system outside the sphere. 


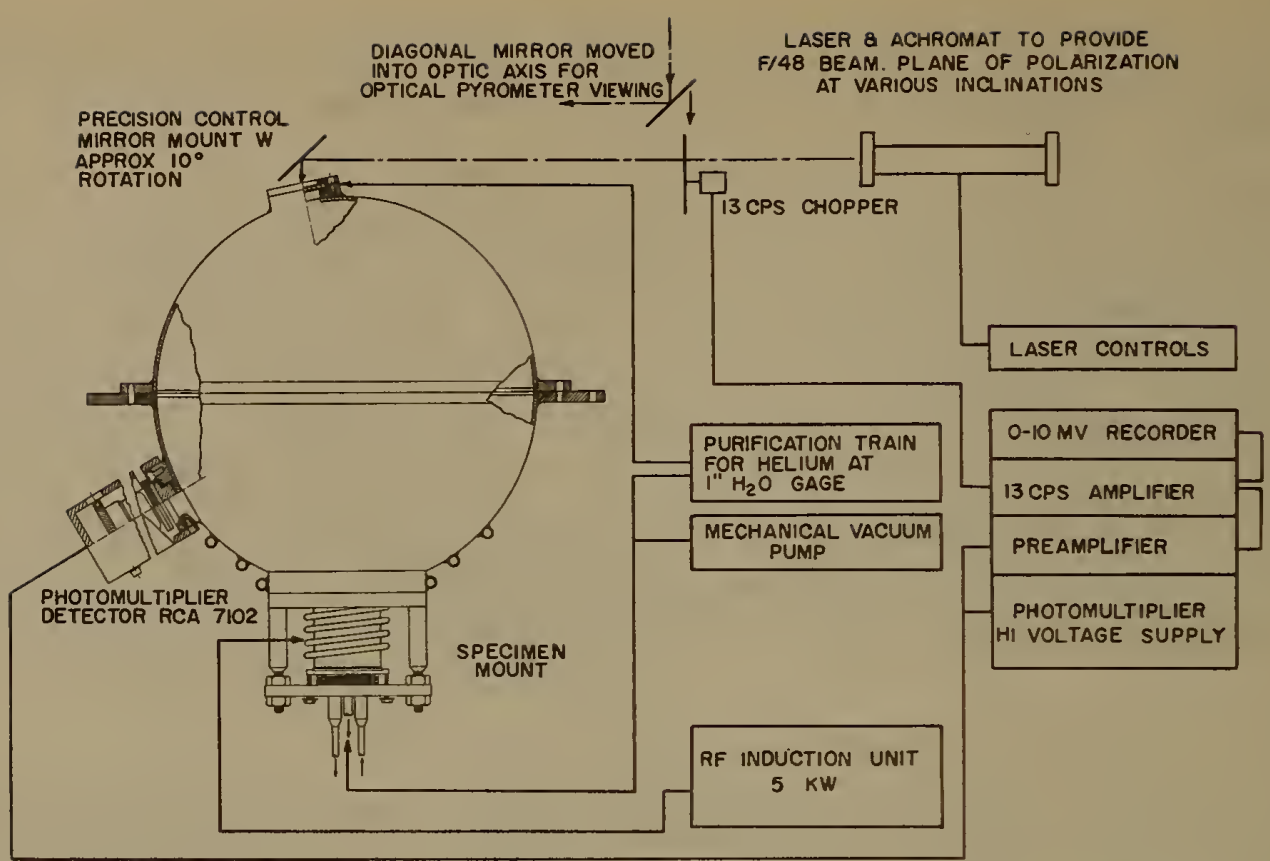

Figure 1. Schematic Diagram of High-Temperature Integrating Sphere Reflectometer, Showing Auxiliary Equipment Required.
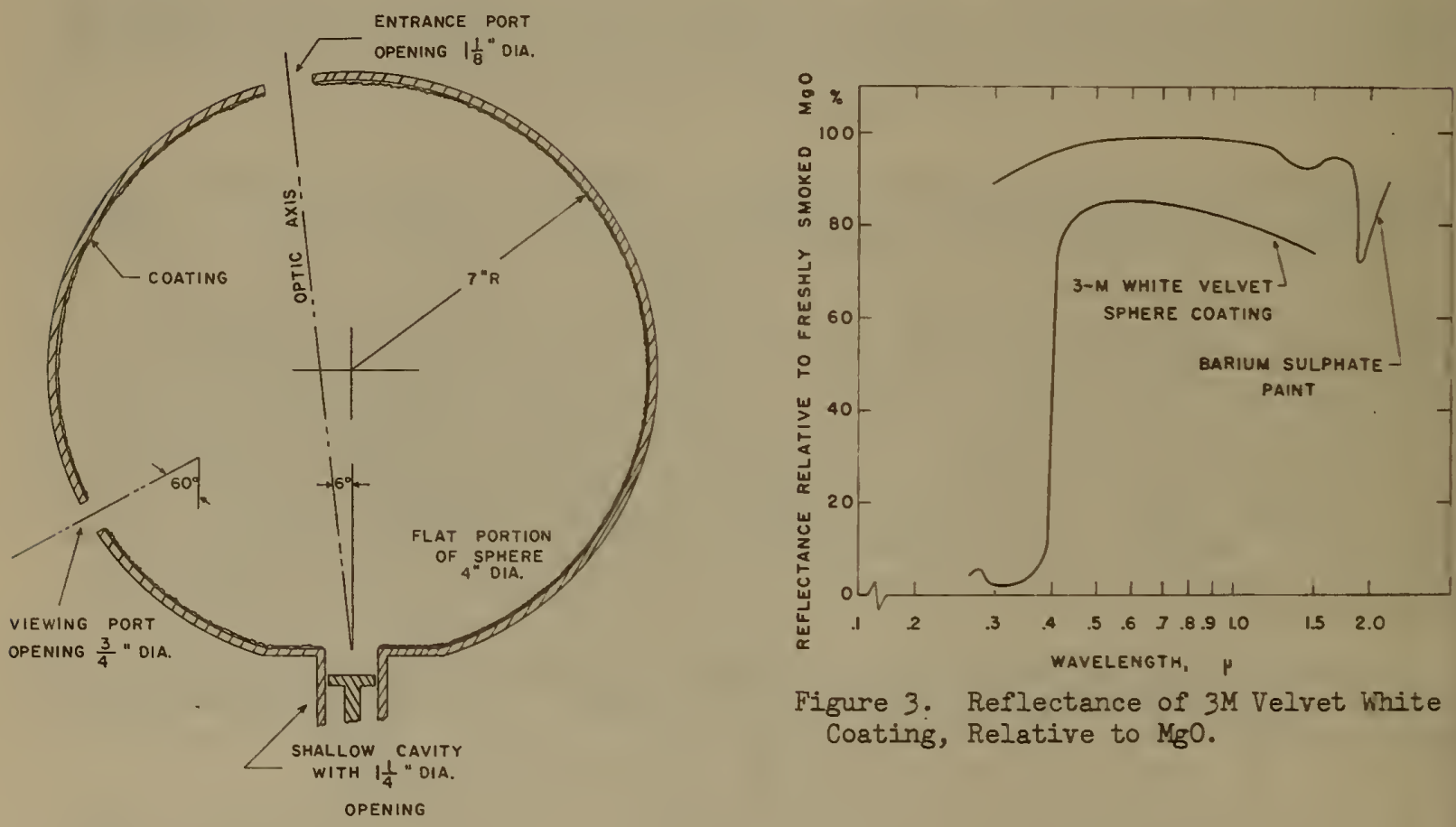

Figure 3. Reflectance of 3M Velvet White Coating, Relative to $\mathrm{MgO}$.

Figure 2. Cross Sectional View of Integrating Sphere, Showing Location of Ports. A shallow cavity is shown at the specimen port. 
A cross-sectional view of the integrating sphere is shown in Figure 2. The sphere coating used for the preliminary measurements was a white primer with a thin top coat of 3M Velvet white paint. 2 ]

The reflectance of the 3M Velvet white sphere coating relative to freshly smoked $\mathrm{MgO}$ is shown in Figure 3. Preliminary goniophotometric measurements indicated that the Velvet coating is a good approximation to a perfect diffuser.

The significant dimensions of the sphere for use with equation (2) are as follows:

$\mathrm{p}_{\mathrm{e}}=$ spherical area of entrance port, $0.994 \mathrm{in.}^{2}$ ( $1-1 / 8 \mathrm{in}$. diameter)

$p_{\mathrm{d}}=$ spherical area of detector port $0.442 \mathrm{in}^{2}$ (3/4 in. diameter)

$p_{\mathrm{S}}=$ effective spherical area of specimen port $1.227 \mathrm{in.}$ ( $1-1 / 4 \mathrm{in}$. diameter)

$\mathrm{S}=$ Total area of sphere wall, $4 \pi \mathrm{R}^{2}, 615.752 \mathrm{in}^{2}$. Radius of sphere is $7.0 \mathrm{in}$.

$S^{\prime}=S-p_{e}-p_{d}-p_{S}$ total reflecting area of sphere wall, 613.089 in. ${ }^{2}$

$\rho=$ reflectance of sphere wall, 0.825 at $0.6328 \mu$ factor C

Substituting the above values in equation (2), we obtain for the sphere, calibration

$$
c=\frac{0.1786-0.00199 p_{s t}}{0.1786-0.00199 p_{s}} \cong 1.00
$$

where $\rho$ stis the reflectance of the standard and $\rho_{\mathrm{s}}$ is the reflectance of the specimen. If $\rho_{\text {st }}$ and $\rho_{\text {st }}$ are equal, $\mathrm{C}=1.00$. If $\rho_{\mathrm{st}}=0.80$ and $\rho_{\mathrm{s}}=0.40$, which is about as large a difference in $\rho_{s}$ and $\rho_{s t}$ as is likely to be encountered, $C=0.9955$. The minimum value of $C$, which will never be attained, would be 0.9889 for $p_{s t}=1.0$ and $\rho_{s}=0$. Because $C$ deviates only slightly from a value of 1.00 , it was neglected in the preliminary checking of the reflectometer.

The detector used at 0.6328 micron was a IP28 photomultiplier. Several different detector configurations were used, as are described later. The signal from the detector was amplified and rectified by standard Perkin-Elmer electronics and recorded by a Leeds and Northrup recording potentiometer. A lead sulphide detector and preamplifier were obtained and are available for use at 1.15 and 3.39 microns.

A low-temperature heater, for heating specimens $12.7 \mathrm{~mm}(1 / 2 \mathrm{in}$. $)$ in diameter to temperatures up to about $800 \circ \mathrm{K}(1000 \cdot \mathrm{F})$ has been designed and built. A schematic drawing of the heater is shown in Figure 4. An important design feature is the provision of holders for specimens of thicknesses of $6.5 \mathrm{~mm}$ (1/4 in.) for nonmetals and $1.6 \mathrm{~mm}$ (0.065 in.) for metals. This heater is for preliminary checking of the equipment. A heater for operation at high temperatures (up to about $2500^{\circ} \mathrm{K}$ or $4000^{\circ} \mathrm{F}$ ) was not constructed during the report period.

\subsection{Calibration of the Integrating Sphere Reflectometer}

\section{4a Iinearity Check}

The linearity of the response of the detector-amplifier-recorder system was checked by means of a rotating disc attenuator with blades that have transmittances of $25.25 \pm 0.14$, $50.00 \pm 0.02$, and $75.10 \pm 0.2$ percent. At 0.6328 micron it was found necessary to insert into the optical path a filter having a trarsmittance of about 0.005 , in order to avoid saturation of the detector and a consequent non-linear response. The filter was located between the integrating sphere and the detector, so that the background radiation from a hot specimen,

2/ The primer was DuPont 65-3010 white, sprayed to a thickness of about $0.075 \mathrm{~mm}(0.003$ in.) and air dried. 


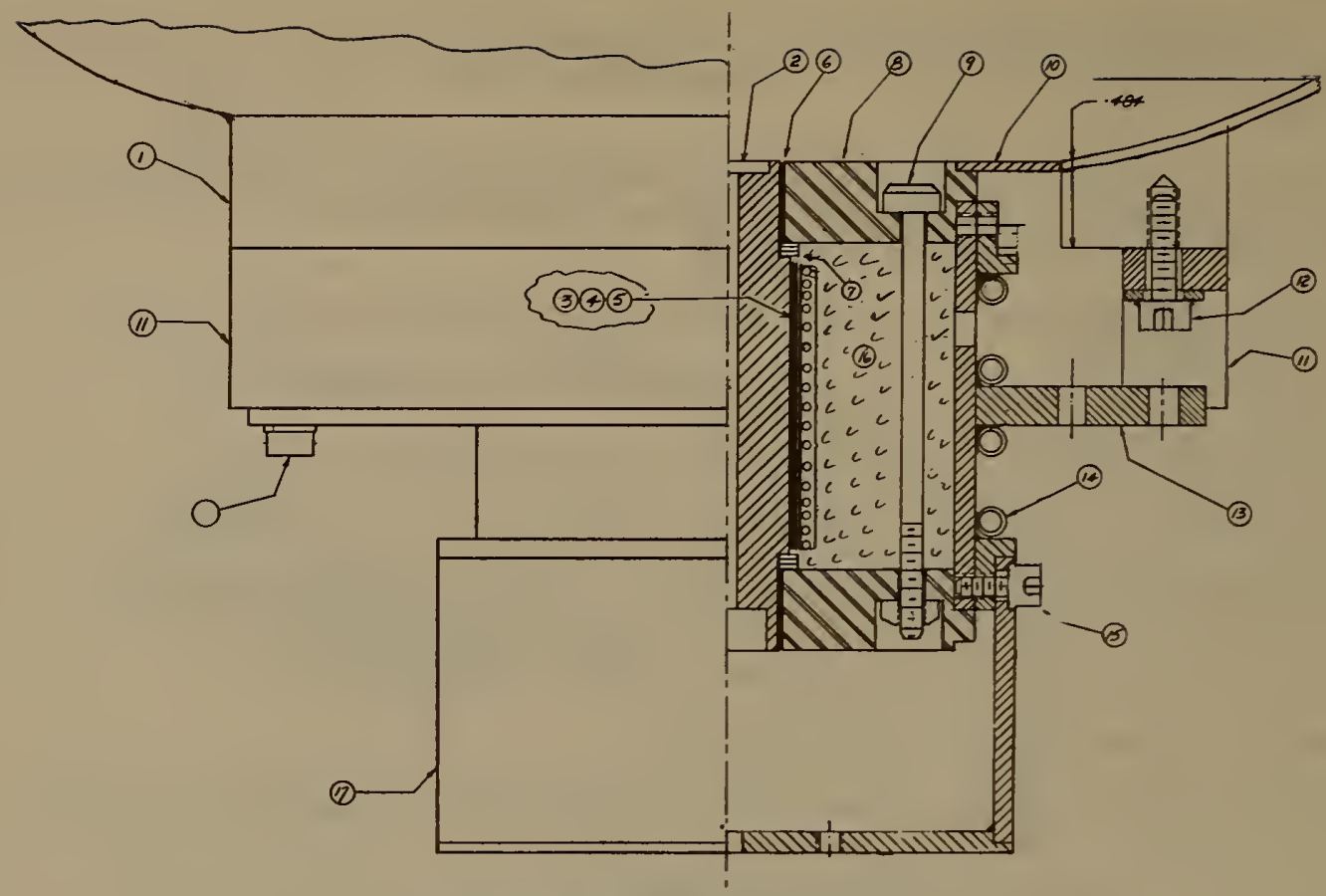

List and Description of Parts

1 Integrating sphere (14" OD) \& 6" OD flange, flange outer flat surface is approximately $1 / 2^{\prime \prime}$ below sphere at heater centerline

2 Heated silver core with cavities for $1 / 2^{\prime \prime}$ diameter specimens of .070" and .250" thicknesses, TC hole diameter .099"

3,4,5 Three layers of GE asbestos paper, $5 \mathrm{ft.} 18$ gauge (.050"D) nichrome wire at $.425 \Omega / f t$., aluminum phosphate cement coating

6,7 Mica spacers and washers, clearance at 6 is .030" clearance at 7 is $3 / 32^{\prime \prime}$

8 End plate, 1/2" thick, transite coat with $\mathrm{SiO}_{2}-\mathrm{Na}_{2} \mathrm{SiO}_{3}$

9 Tie down bolt, $1 / 8^{\prime \prime}$ dia. nominal, 3 required at $120^{\circ}$, steel

10 Sphere wall filler disc, .065 thick, aluminum, coat with $\mathrm{SiO}_{2}$, $\mathrm{Ne}_{2} \mathrm{SiO}_{3}$

11 Alignment boss, alumimum

12 Cap screw 10-32, 3 required at $120^{\circ}$ with washer

13 Body assembly, brass

14 Cooling coils, $1 / 8^{\prime \prime}$ OD tubing

15 Cap screw $8-32,3$ required at $120^{\circ}$

16 Insulation, Fiberfrax

17 Lower cap and cover, brass

Figure 4. Specimen Heater Designed to Heat $12.7 \mathrm{~mm}$ ( $\frac{1}{2}$ Inch) Diamater Specimens to Temperatures Up to About $800 \mathrm{oK}$ ( $1000 \mathrm{oF})$. Specimens of metals $1.6 \mathrm{~mm}$ ( $0.065 \mathrm{inch})$ thick can be accomodated with the heater in the position shown. By reversing the heater, dielectric specimens $6.35 \mathrm{~mm}$ ( $\frac{1}{4}$ inch) thick can be accommodated. 

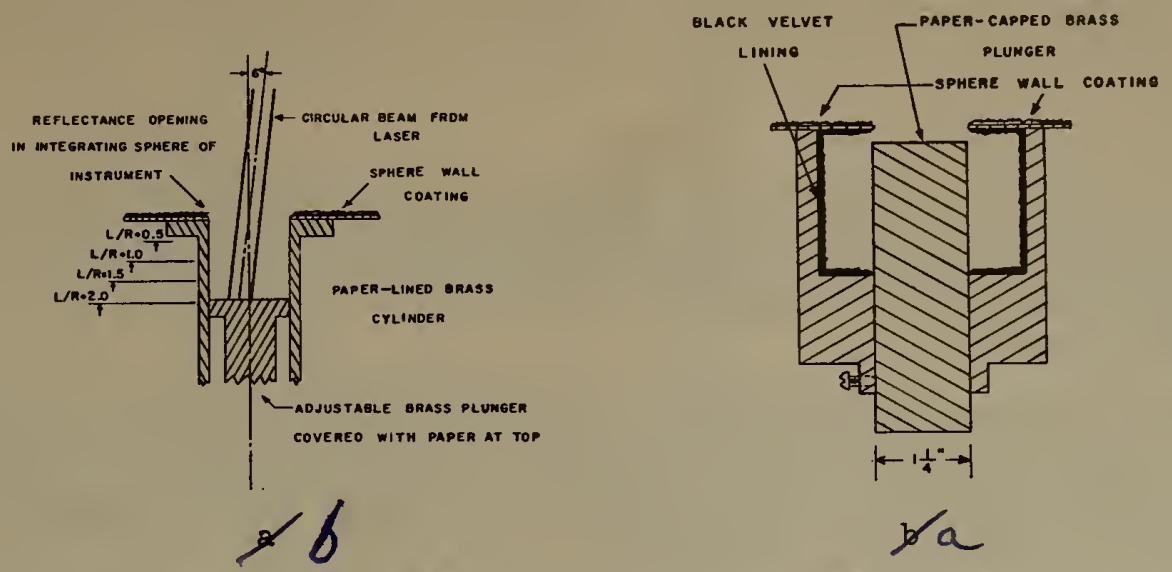

Figure 5. a. First Reflection Cavity. The base of the cavity is a diffuse reflector, but the walls are lined with black velvet, so that only the flux directly reflected by the base is permitted to escape from the cavity opening. b. Iined Cavity. Both base and walls of the cavity reflect diffusely.

The moveable plunger in each cavity permits the depth to radius ratio $(\mathrm{L} / \mathrm{R})$ to be varied from 0.0 to 2.0 . 3M Velvet Jellow paper was used as the diffusely reflecting surfaces.

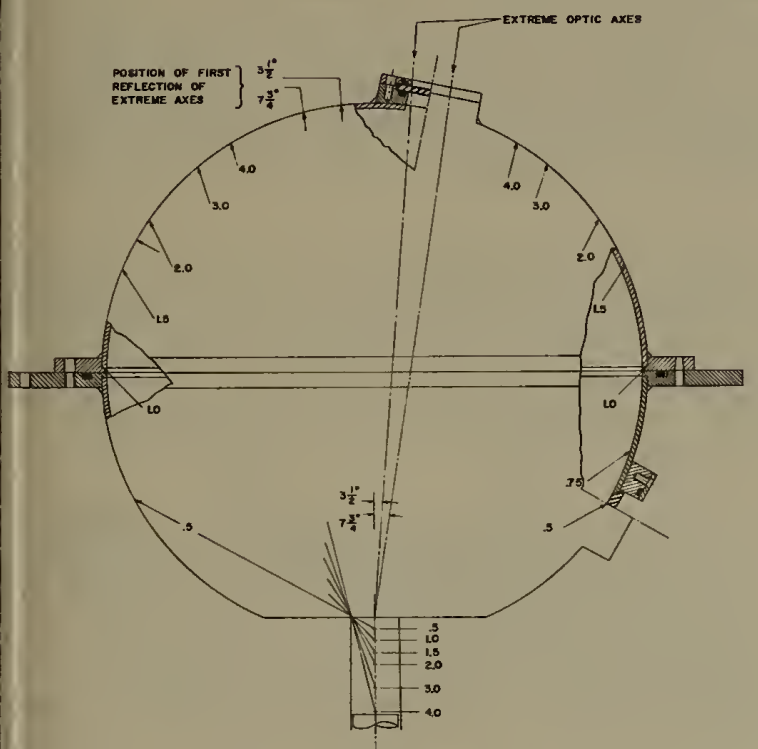

Figure 6. Areas of Sphere Directly Illuminated by Flux Reflected from First Reflection Cavity at Different $L / R$ Ratios.

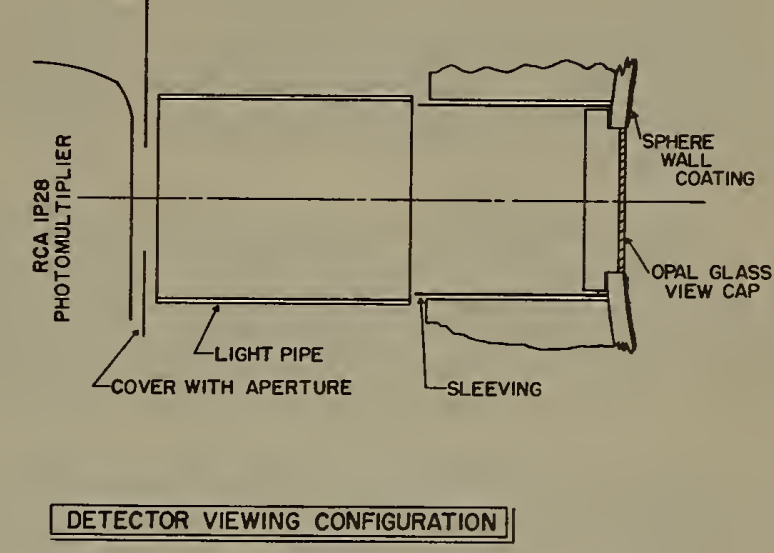

Figure 7. Schematic Diagram of Detector Assembly for Integrating Sphere Reflectometer. 
as well as the reflected radiation would be attenuated. A separate linearity check was made with each experimental set-up, and the data are shown in Tables 1 and 2, (pages 53 and 54 ).

\section{4b First Reflection Cavity Tests}

The procedures used to calibrate the integrating sphere reflectometer were based on previous studies by Kelly and Moore [2]. They verified experimentally the Gouffé [3] equation relating the reflectance of a shallow cavity to its geometry and reflectance of its walls by measuring the reflectance of shallow cavaties of known properties with a General Electric recording spectrophotometer.

If a cavity is used that has a diffusely reflecting bottom and completely absorbing walls, only the flux that escapes through the opening on the first reflection is available for measurement. Such a cavity is shown in Figure 5a. The depth (L) of the cavity is adjustable, so that $L / R$ ratios ( $R$ is the radius of the cavity opening) can be varied from 0.0 to 2.0. The reflectance of the cavity will then be

$$
\rho_{\mathrm{c}}=\rho_{\mathrm{W}} \mathrm{f}
$$

where $\rho_{c}$ is the reflectance of the cavity, $\rho_{W}$ is the diffuse reflectance of the cavity bottom, and $f$ is the diffuse configuration factor between the cavity bottom and opening. For a small spot at the center of the cavity bottom,

$$
f=\frac{1}{1+(L / R)^{2}}
$$

The tests with the first reflection cavity are particularly appropriate for calibration of the integrating sphere reflectometer for two reasons: (1) Equation (4) has been experimentally verified with an instrument of proven reliability, and (2) the geometric distribution of reflected flux varies from a solid angle of $2 \pi$ steradians at $L / R=0$ (cone with central plane angle of $180^{\circ}$ ) to about 0.84 steradian at. $I / R=2$ (cone with central plane angle of $60^{\circ}$ ) as illustrated in Figure 6. Failure of an integrating sphere to give measured $F$ values that agree with the $f$ values computed from equation (4) could result from either deviation of the reflectance of the cavity bottom from perfect diffuseness, or improper viewing conditions for the detector.

Figure 6 shows the areas of the sphere that are directly illuminated by the first reflection cavity at different $L / R$ ratios. It is apparent that at low $L / R$ ratios the detector port is directly illuminated. If the detector views only a portion of the sphere wall, the fraction of the viewed area that is directly illuminated will vary with $L / R$, particularly at high values of $\mathrm{L} / \mathrm{R}$. Consequently the detector response will depend not only on $p_{w} f$ but also on the area of the sphere directly illuminated by the cavity.

The area viewed by the detector can be varied by use of different accessories between the detector and the detector port. Figure 7 is a schematic diagram of the detector assembly, which consists of four parts; (1) an opal glass viewing window, (2) sleeving, which may be either white or black, (3) a light pipe, and (4) a photomultiplier and cover. The space between the detector port and detector can be occupied by any one or more of the elements 1,2 , and 3 above. As an alternative, a series of baffles may be introduced into the passage between the detector and port to reduce the area viewed.

Measurements were made of the reflectance of the first reflection cavity with a paper of $3 \mathrm{M}$ Velvet yellow applied to the base, and with $I / R$ settings of $0.0,0.50,0.75,1.00,1.50$ and 2.00. Several different detector viewing configurations, as illustrated in Figure 8 , were used. The measured reflectance at each value of $L / R$ was divided by the reflectance at $L / R=0$ to obtain the $F$ values shown in Table 1 . The difference between the computed values, $f$, and measured values, F, is plotted as a function of $L / R$ in Figures 9, 10, and 11.

The data show that the configurations with the opal glass viewing cap (trials 105 and 106), which enable the detector to view the entire sphere wall, give high values of $F$. The configurations that restrict the field of view with collimators to a small area opposite the detector (trials 108, 109, 110), and with black sleeves only (trials 104 and 107), give low 


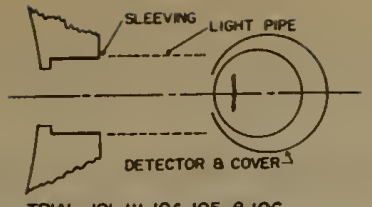

TRIAL 1O, III, 10, 105, a 106

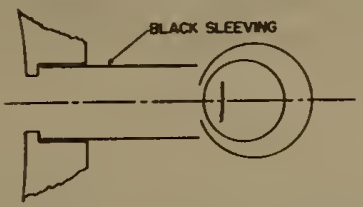

TRIAL 107

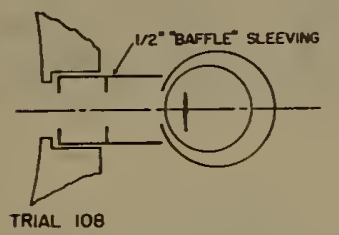

Figure 8. Detector Configurations Used in First Reflection Cavity Tests.

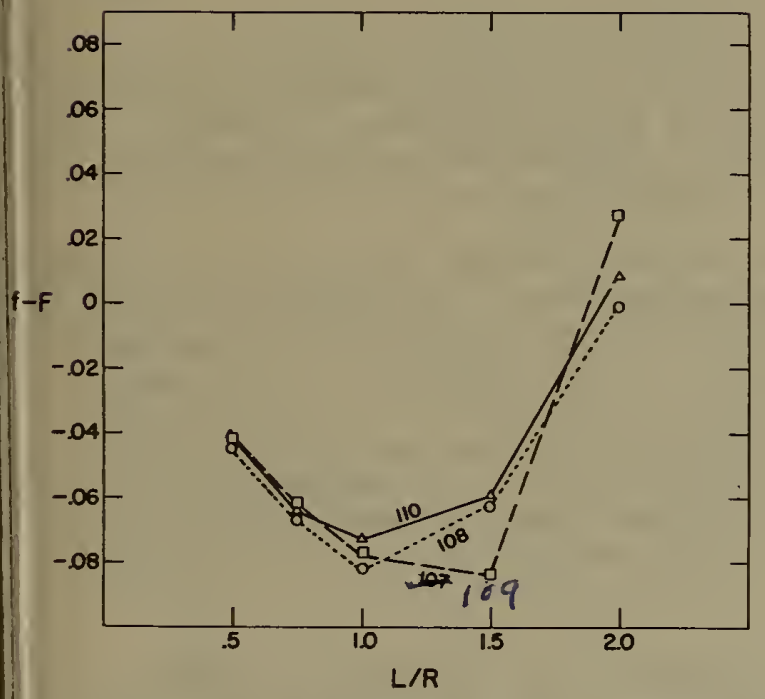

Figure 10. Difference Between Measured $F$ and Computed $f$, Plotted as a Function of $L / R$, for Detector Configurations With Collimators, 108 - $\frac{1}{2}$-Inch Collimator, 0.075 Inch Beam; 109 - $\frac{1}{4}$-Inch Collimator, 0.075 Inch Beam; 110 - Same as 109, Except EMI 5685 Photomultiplier Instead of IP28.

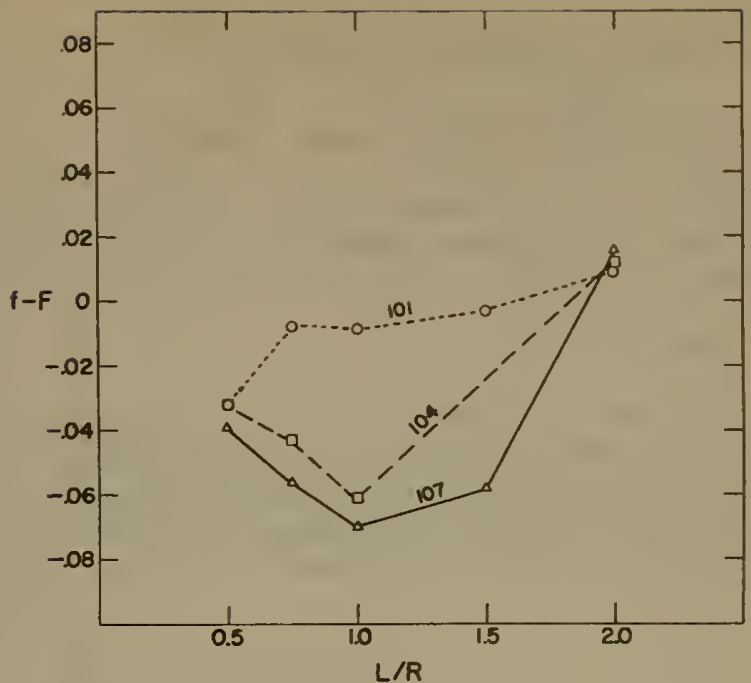

Figure 9. Difference Between Measured F and Computed $f$, Plotted as a Function of $\mathrm{L} / \mathrm{R}$, for Detector Configurations With Black Sleeving, 101-Black Sleeve with Light Pipe; 104-Black Sleeve Only; 107-Long Black Sleeve, 0.075 Inch Beam.

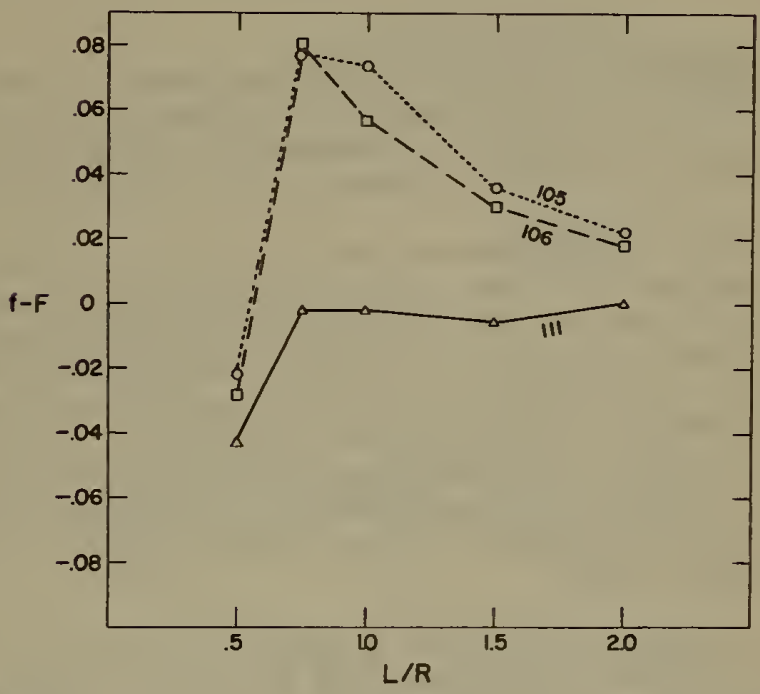

Figure 11. Difference Between Measured $F$ and Computed $f$, Plotted as a Function of $L / R$, for Detector Configurations with White Sleeving, 105 - White Sleeve, Opal Glass View Cap and light Pipo; 106 - Same as 105, but 0.075 Inch Beam; 111 - White Sleeve, Iight Pipe, 0.075 Inch Beam. 
values of F. Best agreement was obtained in trials 101 (black sleeve, light plpe) and 111 (white sleeve, light plpe, 0.075 in. beam).

The results of these tests suggested that the integrating sphere was only marginalif suitable for the intended use. It has not been established whether this is due to (I) the relatively low reflectance of the sphere coating, about 0.82 as applied, (2) variations in reflectance over the surface of the sphere, (3) the location of the detector port, or (4) the lack of baffling between the detector port and specimen.

\section{4c Iined Cavity Tests}

If a cavity is used which has diffusely reflecting bottom and walls, as illustrated in Figure 5b, a smaller fraction of the incident flux will be absorbed, and the reflected flux will be more nearly diffuse. The experimental data of $\mathrm{Kelly}$ and Moore [2] agreed well with those computed from the Gouffe equation for the reflectance of the cavity, $\rho_{c}$, in terms of the $L / R$ ratio and the reflectance of the cavity wall $8, \rho_{H}$.

$$
\rho_{C}=1-\frac{\left(1-\rho_{H}\right)\left[1+\rho_{H}\left(\frac{a}{A}-\rho\right)\right]}{1-\rho_{W}\left(1-\frac{a}{A}\right)}=\frac{1-\rho_{H}\left(1-\frac{a}{A}\right)}{\left.\rho_{H} \frac{a}{A}\right)}
$$

where $\frac{a}{A}=\frac{1}{2(I+I / R)}$ and $f=\frac{1}{1+(I / R)^{2}} \cdot \frac{a}{A}$ is the ratio of the opening of the cavity to the total wall area of the cavity, opening included, and $f$ is the fraction of the incident flux leaving the cavity on the first reflection (equation 4 ). Setting $H=\rho_{c} / \rho_{W} g I v e s$

$$
\rho_{H}=\frac{H-f}{(H-\rho)+\frac{Q}{A}(I-H)}
$$

Equations (5) and (6), like equation (4), are based on center-point illumination and on the assumption that the walls and bottom of the cavity are perfectiy diffuse reflectors. The laser beam gives a good approximation of the center point illumination, as the dismeter of the beam was only about $1.9 \mathrm{~mm}$, while the diameter of the cavity was $32 \mathrm{~mm}$.

If the values measured with the first reflection cavity agree with the computed $f$ values, the lined cavity values should yleld accurate absolute reflectances. The effect of uncertainties in $L / R$ and the measured reflectance ratio, $Q_{c} / Q_{w}$, on the accuracy of the computed absolute reflectance, $P_{W}$, is shown in Flgures 12 and 13. The data indicate that the method should give reasonable precision, even for reflectances as low as 0.50 . The method is convenient, since a cavity is easily prepared. If the reflectometer gives accurate values of $F$ with the first reflection cavity and $\rho_{H}$ with the lined cavity, $1 t$ can be assumed that accurate values will be obtained with diffusely reflecting spscimens of almost any geometric distribution of reflected flux, provided there is negligible specular reflection back out the entrance port.

The measured values of $\mathrm{F}$ obtained in trials 101, 104, and 111, reported above, gave the best agreement with computed $f$ values. Hence evaluation of $\rho_{W}$, the absolute reflectance of the 3M Velvet yellow and green papers, was attempted by the shallow cavity approach, using these same detector configurations. The data for the yellow paper are reported in Table 2 as trials 102, 103, and 112, with the viewing configurations reported in trisls 101, 104, and 112, respectively, and for the green psper as trials 301 for the first reflection cavity and 302 for the lined cavity, using the same viewing conflguration as in trial 171 . The data were reduced by use of the following equation.

$$
\rho_{W}=\frac{H-F}{\left(I-\frac{a}{A}\right)(H-F)+\frac{a}{A}(I-I)}
$$




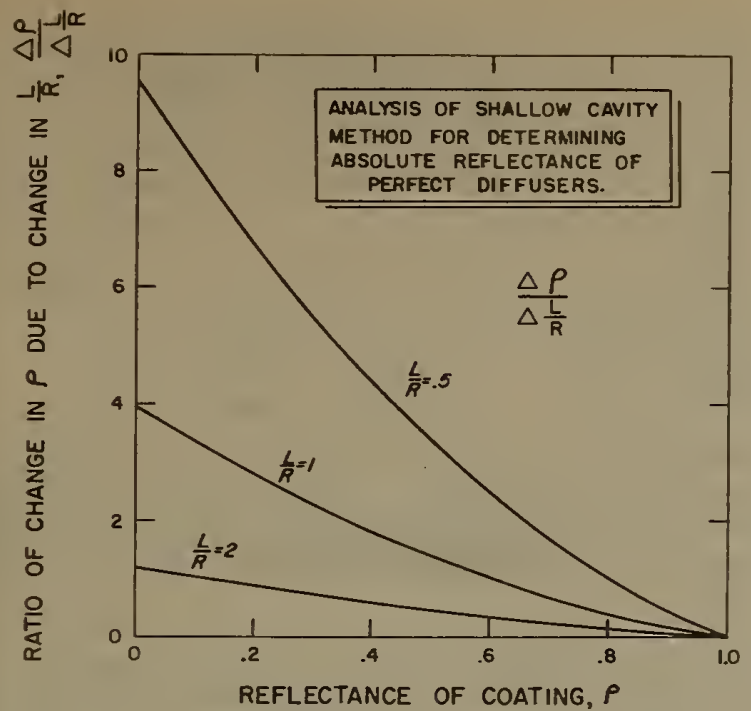

Figure 12. Effect of Uncertainty in $I / R$ on Accuracy of $\rho_{W}$ Evaluated by Shallow Cavity Method.

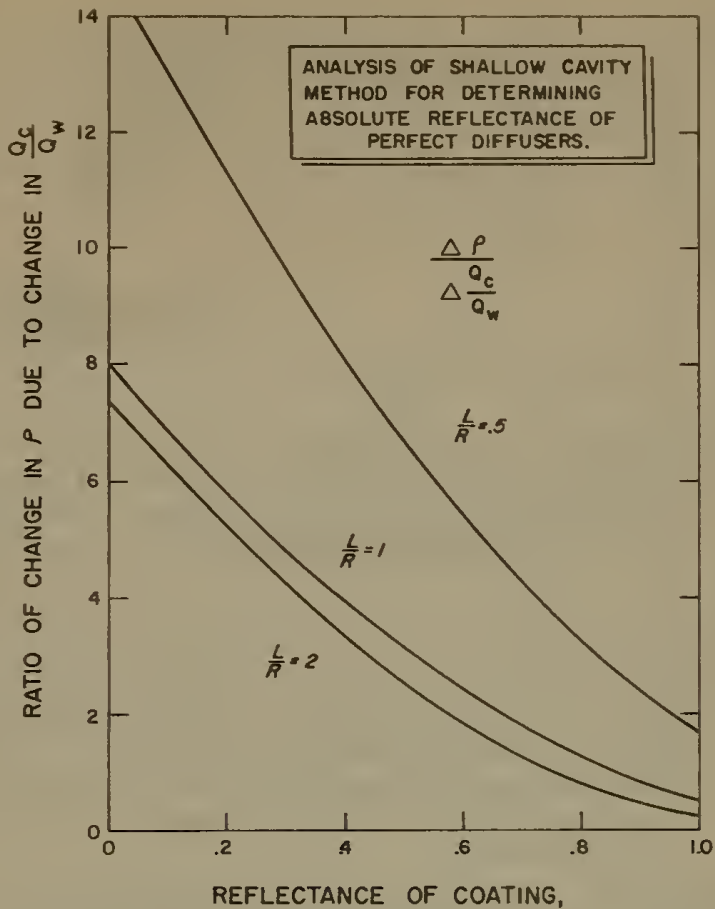

Figure 13. Effect of Uncertainty in Measured Reflectance Ratio Qc/Qw, on Accuracy of $\rho_{\mathrm{W}}$ Evaluated by Shallow Cavity Method.

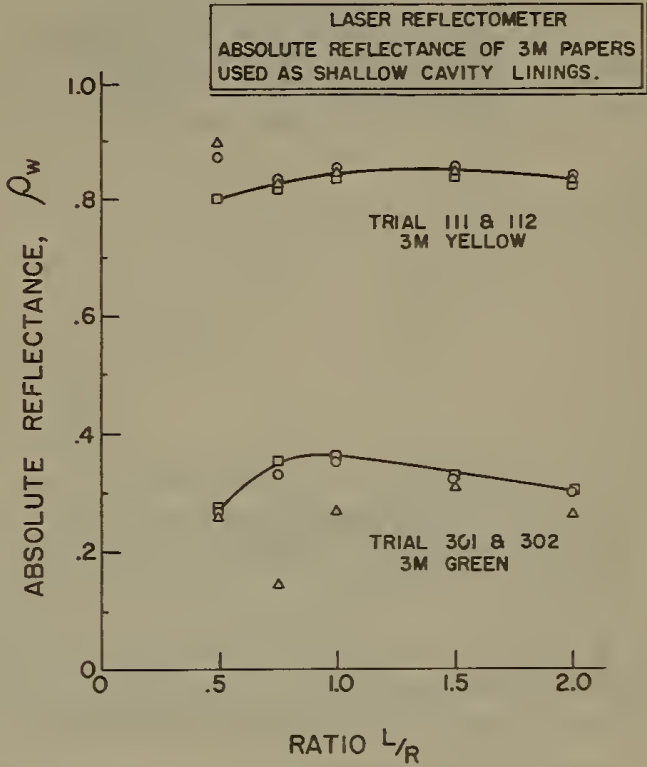

$\triangle$ THEORETICAL $f$ VALUES, METHOD I - EXPERIMENTAL F VALUES, ALL REFLECTIONS, METHOD 2

- EXPERIMENTAL F IST REFLECTION, OTHERS $f$, METHOD 3

Figure 15. Absolute Reflectance of 3M Velvet Yellow Paper, Computed from Data of Trials 111 and 112 , Top, and of 3M Velvet Green, Computed from Data of Trials 301 and 302, Bottom, Plotted as Functions of $\mathrm{L} / \mathrm{R}$ Ratio. 


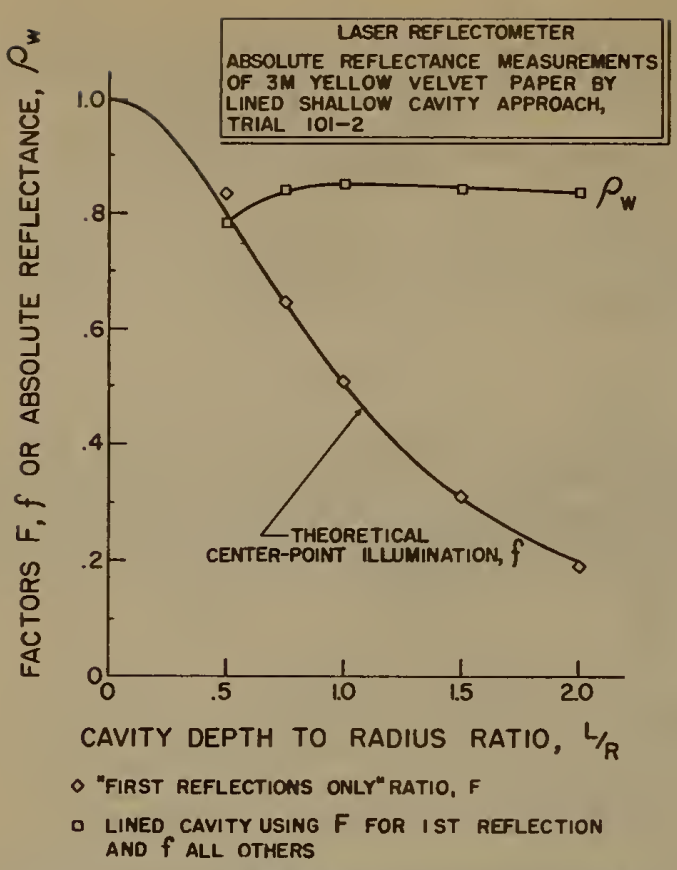

Figure 16. Experimentally Determined F Values and Absolute Reflectance $\rho_{W}$, of 3M Velvet Yellow Paper, Computed by Use of Equation (7), Trials 101-102.

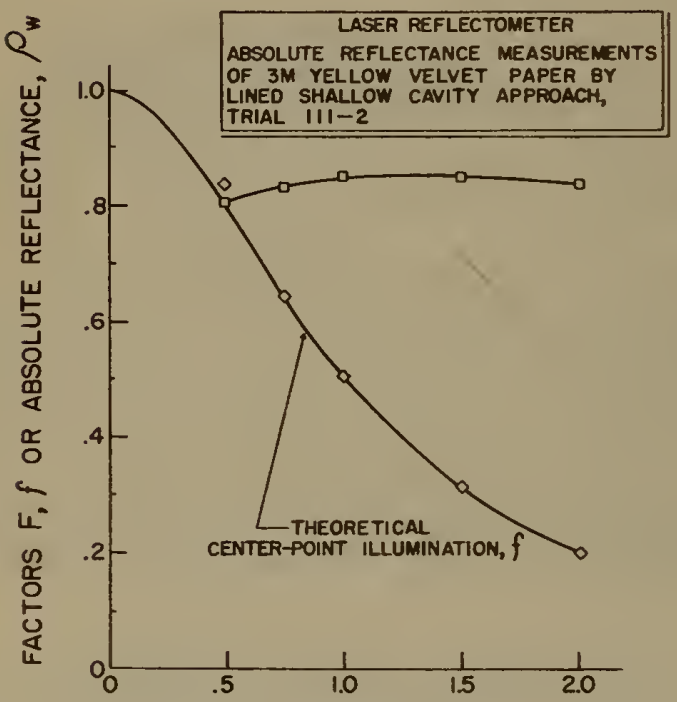

CAVITY DEPTH TO RADIUS RATIO, L/R

- FIRST REFLEcTIONS ONLY, F

- LINED CAVITY USING F FOR IST REFLECTION AND $f$ ALL OTHERS

Flgure 18. Experimentally Determined F Values and Absolute Reflectance, $\rho_{W}$, of 3M Velvet Yellow Paper, Computed by Use of Equation (7), Trials 111-112.

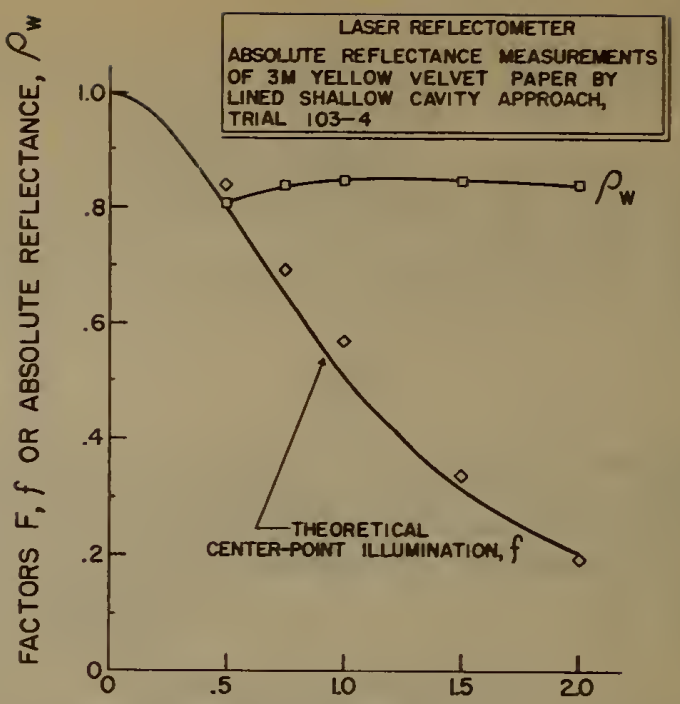

CAVITY DEPTH TO RADIUS RATIO, L/R - fiRSt REFLECTIONS ONLY, F

- LINEO CAVTTY USING $F$ FOR IST REFLETIION AND $f$ ALL OTHERS

Figure 17. Experimentally Determined F Values and Absolute Replectance, $p_{H}$, of 3M Velvet Yellow Paper, Computed by Use of Equation (7), Trials 103-104.

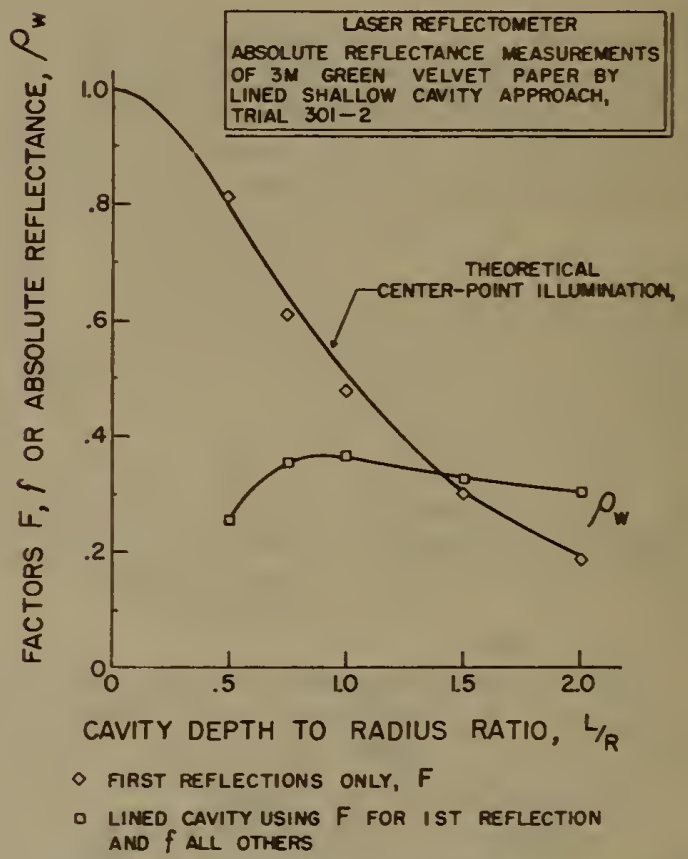

Figure 19. Experimentally Determined F Values and Absolute Reflectance of $3 \mathrm{M}$ Velvet Green Paper, Computed by Use of Equation (7), Trials 301-302. 
Equation (7) is a modification of equation (6) in which a distinction is made between $F$ and

$F$ is used in the equation where flux from the first reflection only is considered, and $f$ where the flux from multiple reflections is involved. Ideally, $F=f$ for a completely diffuse cavity wall and an ideal integrating sphere reflectometer. However, the measured $F$ deviated somewhat from the computed $f$ in nearly every case. H, F, $f$ and a/A are as previously defined.

The data were reduced by three procedures:

(1) Computed values of $f$ were used for both $f$ and $F$.

(2) Measured values of $F$ were used for both $f$ and $F$.

(3) Measured values of $F$ and computed values of $f$ were used where applicable.

The results of the computations are given in Table 3 for the original data obtained in trials 101-102, 103-104, 117-172 and 301-302, and are plotted as functions of L/R ratio in FIgures 14 and 15. The results obtained by procedure 3, together with the corresponding values of $f$ and $F$, are plotted as functions of $L / R$ ratio in Figures 16, 17, 18 and 19.

Procedure 3 appears to give the most consistent results. The over-all scatter is least, and the three curves for $\rho_{\mathrm{W}}$ of the $3 \mathrm{M}$ Velvet yellow paper computed by this procedure nearly coincide. The reflectance of the $3 \mathrm{M}$ Velvet paper, relative to $\mathrm{MgO}$, was 0.850 at $0.632 \mu$ as measured with the General Electric recording spectrophotometer. Converting this reflectance to absolute on the basis of the absolute reflectance of $\mathrm{MgO}$ of 0.985 at $0.632 \mu$ as reported by Edwards et al [4], we obtain a value of 0.837 . The corresponding value for the reflectance of the $3 \mathrm{M}$ Velvet green paper, relative to $\mathrm{MgO}$, is 0.385 at $0.632 \mu$, which converts to 0.379 on an absolute scale.

AII of the absolute reflectances computed from data taken at $L / R$ values of 0.5 appear to be in error. The values are high as computed by methods 1 and 2 , and 10 w as computed by method 3. There is also a tendency for the absolute reflectances computed from data taken at $L / R$ values of 2.0 to be somewhat low. The data computed by procedure 3 appear to be the most consistent. The 12 values obtained by this procedure at $\mathrm{L} / \mathrm{R}$ values of $0.75,1.0$, 1.5 and 2.0 , average 0.842 with a standard deviation of 0.006 , which is an excellent check of the value of 0.838 computed from the reflectance measured with the General Electric recording spectrophotometer. The corresponding average value for the absolute reflectance of the $3 \mathrm{M}$ Velvet green paper at $0.632 \mu$, however, is 0.339 , which is significantly lower than the value of 0.380 computed from the reflectance measured with the General Electric recording spectrophotometer.

The flux from the first reflection, $I_{O} \rho_{W} f$, is a major portion of the total flux reflected from a shallow cavity. $K$, the fraction of the total flux reflected on the first reflection, can be computed for a perfect diffuser as

$$
K=\frac{\rho_{W} f}{\rho_{c}}=\frac{f-\rho_{W} f\left(1-\frac{\varepsilon}{A}\right)}{f-\rho_{W}\left(\frac{a}{A}-f\right)}
$$

The fraction $K$ is plotted as a function of wall reflectance, $\rho_{w}$, for different $I / R$ ratios in Figure 20. The data show that for low $\rho_{W}, K$ becomes very large, that $K$ decreases with increasing $I / R$ for any $\rho_{W}$, and that $K$ decreases markedly with an increase in $I / R$ at high $\rho_{W}$. As a first approximation, the fraction $K$ of the flux reflected from a shallow cavity ${ }^{*}$ will be distributed uniformiy over the solid angle subtended by the cavity opening from the center of the incident beam, and the fraction ( $I$ - K) of the flux will be distributed uniformly over the entire hemisphere. Hence the geometric distribution of the flux reflected from a shallow lined cavity will be intermediate between that from a first reflection cavity and that of a perfect diffuser.

No detailed explanation of the shape of the curves in Figures 14 through 19 will be attempted at this time, The data are preliminary, and are subject to errors that will be evaluated as the work progresses.

2.4d Appended Sphere Tests

The absolute reflectance of a coating material can also be evaluated by the appended sphere method.3/ The diffusely reflecting coating to be evaluated is applied to the inside

3/ Much of the theory for the appended sphere method was supplied by David D. Goebel of the Photometry and Colorimetry Section of the National Bureau of Standards. 


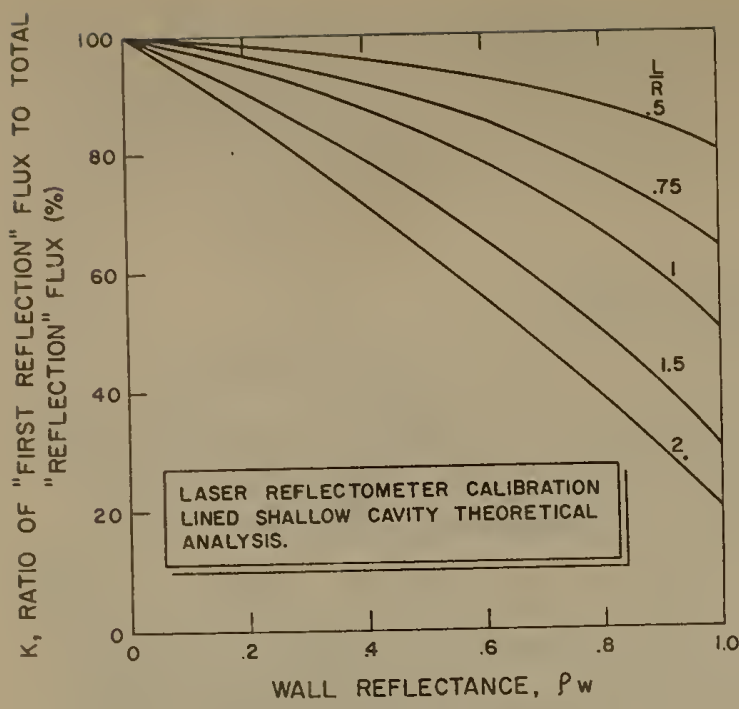

Figure 20. Ratio of First Reflected Flux to Total Flux, K, Reflected by a Shallow Cavity for Different $L / R$ Ratios, as a Function of Wall Reflectance, $\rho_{W}$.

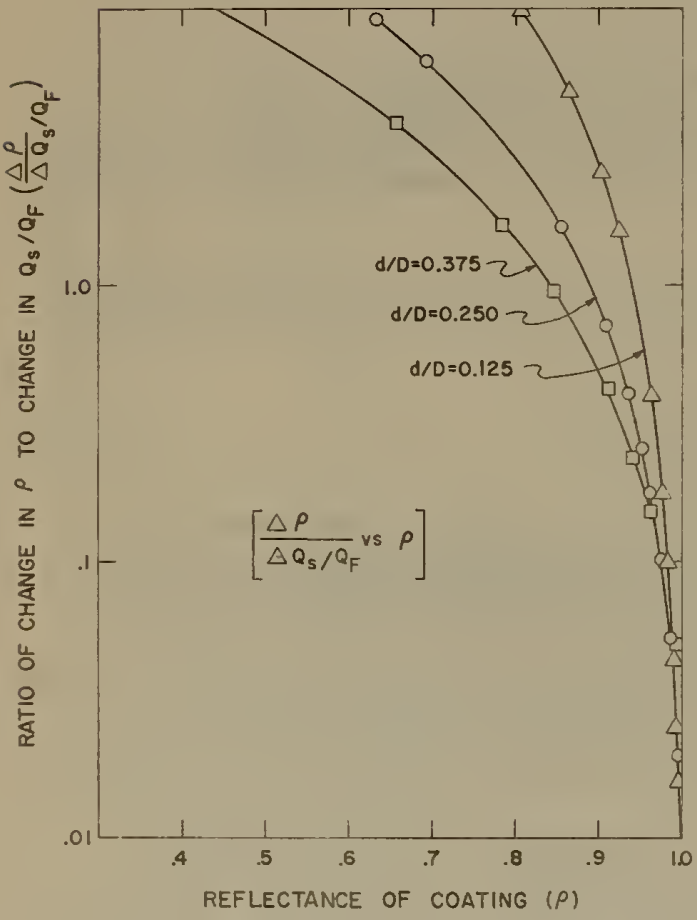

F1gure 22. Effect of Change in Measured Reflectance Ratio, $Q_{S} / Q_{F}$, on Reflectance by the Appended Sphere Method, Plotted as a Function of Reflectance, $\rho$, for Different Sphere Geometries, d/D.

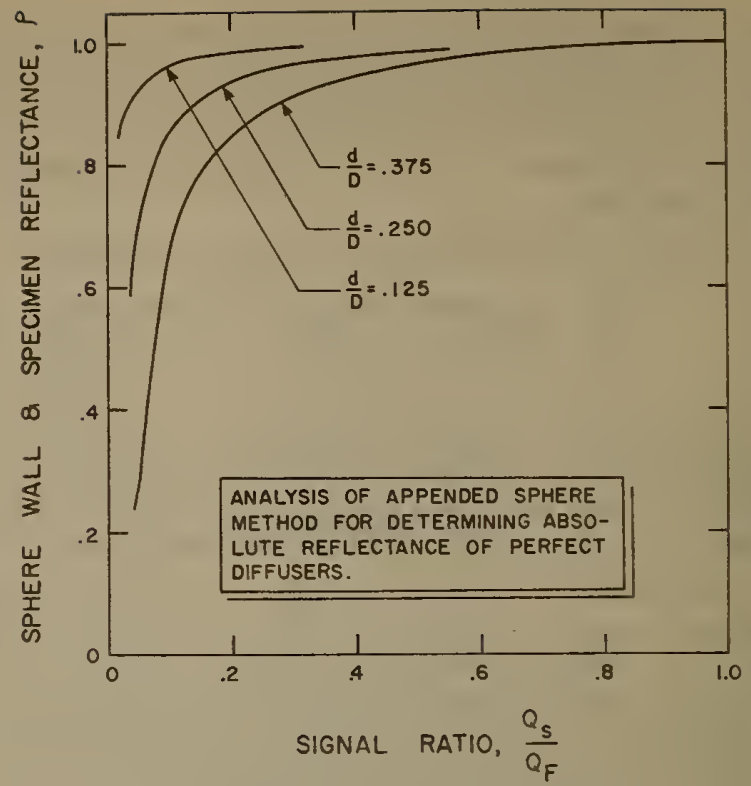

Figure 2l. Relation of Reflectance, $p$, to Measured Reflectance Ratio, $\mathrm{Q}_{\mathrm{S}} / \mathrm{QF}_{\mathrm{F}}$, in the Appended Sphere Method, for Different Sphere Geometries, $d / D$.

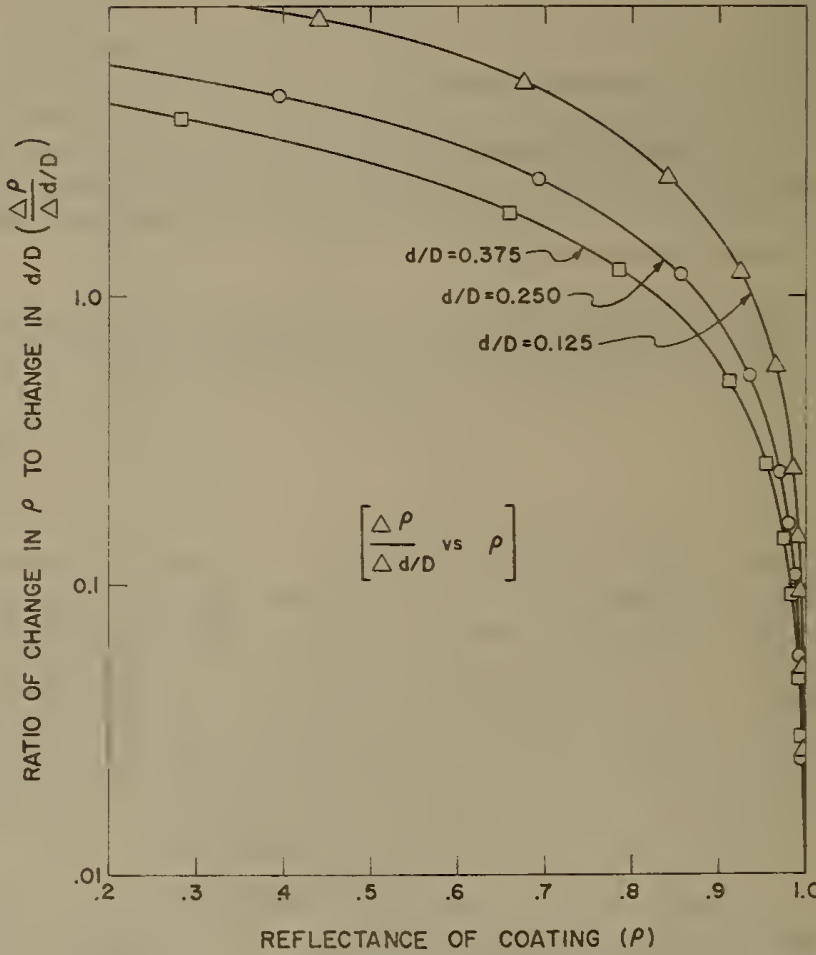

Figure 23. Effect of Change in Sphere Geometry, d/D on Reflectance by the Appended Sphere Method, Plotted as a Function of Keflectance, $p$, for Different Sphere Geometrles. 
surface of a small sphere having a single hole of the same diameter as the specimen port on the integrating sphere, and to a flat specimen of any convenient size. The reflectance of the sphere, $Q_{S}$, and of the flat specimen, $Q_{f}$, are measured. These reflectances can be measured relative to any convenient standard or to each other.

The absolute reflectance of the coating, $\rho$, is computed from the measured reflectance ratio, $Q_{S} / Q_{f}$, by use of the following equation:

$$
p=\frac{1-\frac{q}{Q_{S} / Q_{f}}}{1-q}
$$

where $\mathrm{q}$ is the fraction of incident flux that leaves the sphere on the first reflection, computed as:

$$
q=\frac{1}{2}\left[1-\sqrt{1-\left(\frac{d}{D}\right)^{2}}\right]
$$

where $d$ is the diameter of the opening in the appended sphere, and $D$ is the diameter of the appended sphere.

The relation of $\rho$ to $Q_{S} / Q_{f}$ for different values of $d / D$ is shown in Figure 21 . These curves indicate that as the reflectance $p$ of the coating decreases, the reflectance ratio $Q_{S} / Q_{f}$ decreases very rapidly, particularly for smaller $d / D$. The conclusion is that the methôd is not very precise for measuring absolute reflectance of low-reflectance coatings. However, the appended sphere method does have inherently high accuracy for evaluating the absolute reflectance of highly reflecting diffuse coatings, as is shown in Figures 22 and 23. Figure 22 shows the uncertainty in $p$ produced by an uncertainty in the measured reflectance ratio, $Q_{S} / Q_{f}$, for different $d / D$ ratios as a function of $\rho$. Figure 23 shows the corresponding uncertainties in $\rho$ due to uncertainties in $d / D$. As an example, for $d / D$ of 0.250 , if $Q_{S} / Q_{f}$ is uncertain by $I$ percent and $d / D$ is also uncertain by $I$ percent, the uncertainty in $\rho$ at 0.98 is only 0.2 percent. However, if $\rho$ is only 0.80 , the corresponding uncertainty in $p$ increases to about 4.4 percent.

The appended sphere method differs from the shallow cylindrical cavity method in two important respects: (1) there is no theoretical verification for the appended sphere method, and (2) the flux reflected from the appended sphere has a geometric distribution that more closely approximates that from a perfect diffuser.

Several spheres were prepared for use with the appended sphere method, but no measurements were completed during the report period.

\subsection{Relative Reflectance Measurements}

The laser-source integrating sphere reflectometer should be suitable for making relative reflectance measurements, even if errors are present in the measured absolute reflectance. The 3M Velvet yellow paper was selected as a comparison standard, and the relative reflectance of a number of materials was measured. The data are given in Table 4; and compared to similar values obtained on the same specimens with the General Electric recording spectrophotometer or the Cary Model 14 spectrometer, both at $0.632 \mu$. The data are preliminary, and no detailed analysis will be attempted at this time.

\subsection{Modifications to Reflectometer}

Because of the somewhat disappointing results obtained with the integrating sphere reflectometer as originally designed and with the $3 \mathrm{M}$ Velvet white coating, the sphere was modified by adding a second detector port, $135^{\circ}$ from the specimen port in the plane of the entrance port. Because of the location of the flange connecting the two halves of the sphere, it is also possible to rotate the lower half of the sphere so that the original detector port is in a plane normal to the plane of the entrance port. The 3M coating was replaced by Baso, coating, having appreciably higher reflectance. It is expected that the new sphere coating and detector port will appreciably reduce the errors that were present in the original design. 


\subsection{Background - Need for Instrument}

Normal spectral emittance measurements of specimens at and near room temperature are not easy to make. The total amount of energy emitted at such temperatures, even by a blackbody radiator, is small, and when further attenuated by a monochromator, the amount is so small that special techniques are required to measure it. On the other hand, energy considerations do not seriously affect reflectance measurements. As long as the temperature of the specimen remains approximately constant, the fraction of the incident flux that is reflected is independent of the incident flux density. Hence we can measure spectral reflectance and compute spectral emittance by use of Kirchhoff's law.

There is a more urgent and specific need for spectral reflectance data in the current program. The laser-source reflectometer described in Section 2 of this report measures reflectance only at those few wavelengths at which continuous wave lasers operate, but provides some spectral reflectance data at high temperatures. It is fundamentally incapable of providing the continuous spectral reflectance curve needed for heat-transfer computations.

Available data indicate that, while spectral emittance or reflectance of a material at any one wavelength may change significantly with temperature, the general shape of the spectral reflectance or emittance curve does not, provided the material does not undergo any phase change or irreversible change in physical properties as a result of heat treatment. Hence it is possible to construct a spectral reflectance or emittance curve for material from accurate data obtained at a few wavelengths with the laser source reflectometer, by interpolating values at intermediate wavelengths on the basis of the shape of the room temperature curve. For the reflectance to be useful for computing normal spectral emittance by Kirchhoff's law, or for interpolation of laser-source reflectometer data, it must be measured under equivalent optical conditions -- normal illumination and hemispherical viewing, or the optical equivalent diffuse illumination and normal viewing.

A number of different instruments have been designed to measure reflectance under conditions approximating normal illumination and hemispherical viewing. These include the Coblentz. hemisphere, which approximates normal illumination and hemispherical viewing, the integrating sphere reflectometer, which can be used to approximate either of the conditions mentioned above, and the hohlraum reflectometer and Janssen's modification of the Coblentz hemisphere reflectometer, which approximate diffuse illumination and hemispherical viewing. Each of thes has advantages and disadvantages, but none was deemed entirely suitable for our measurements.

In an attempt to design an instrument that would provide data of the desired reliability, at wavelengths out to at least 15 microns, the ellipsoidal mirror reflectometer was conceived. Basically, the instrument is a modification of the Coblentz hemisphere, in which an ellipsoida mirror replaces the hemispherical mirror. A narrow beam of radiation from the monochromator is focused through a small hole in the ellipsoidal mirror onto the specimen, which is located at the first focus of the ellipsoid. The ellipsoidal mirror focuses the radiation reflected by the specimen onto the detector, which is located at the second focus of the ellipsoid, some 17 inches below the specimen.

Advantages of the ellipsoidal mirror reflectometer include:

A. The detector views a solid angle having a central plane angle of only about $45^{\circ}$ rather than a hemisphere, as in the Coblentz method, hence errors due to variations in angular sensitivity of the detector are reduced.

B. The ellipsoidal mirror produces an image that is much freer from optical aberrations than is the case with a hemispherical reflector.

C. The separation of sample and detector by some 17 inches permits the specimen to be heated or cooled without adversely affecting the detector.

D. Diaphragms at the first focal plane can be used to selectively block out or pass radiation reflected into known solid angles, hence measurements can be made of the geometric distribution of reflected radiation. 
E. The reflectance of the mirror coating remains high throughout the infrared. Most integrating sphere coatings have low reflectance beyond about $2.5 \mu$.

F. The instrument can be used for absolute as well as comparative reflectance measurenents.

The principal disadvantages of the ellipsoidal mirror reflectometer are:

A. The specimen and its support block a small portion of the optical path at the first focal plane.

B. The mirror has a linear magnification factor of about 5, hence a large-area detector is required, and the illuminated area on the specimen must be kept small.

\subsection{Previous Work}

3.2a Design of Equipment

Equipment for measuring spectral reflectance under conditions approximating normal illumination and hemispherical viewing, over the wavelength range of approximately 1 to 15 microns, was designed and built, as described in WADC TR 59-510 pts. II [52]and III r 53].

The reflectometer consisted of six distinct parts: (1) a source assembly, (2) a monochromator, (3) transfer optics, (4) an ellipsoidal mirror, (5) a detector, and (6) an amplifier and recorder. The source assembly, (1), consisted of a silicon-carbide element, a chopper and a pair of mirrors, one plane and one spherical, which focused the chopped flux from the hot element onto the entrance slit of the monochromator (2). The monochromator, (2), which was equipped with a sodium chloride prism, dispersed the flux. The transfer optics, (3), directed and focused the chopped and dispersed flux, through a small hole in the ellipsoidal mirror, onto the specimen, which was located at the first focus of the ellipsoid. The ellipsoidal mirror (4) collected the flux reflected by a specimen located at its first focal point and focused it on the detector located at the second focal point. A Golay-cell detector (5) was mounted on a device which permitted it to be positioned at either the first focal point or second focal point of the mirror. The amplifier and recorder (6), made a permanent record of the amplified signal from the detector. The reflectometer was designed to measure absolute reflectance by the following procedure.

The incident flux was measured with the detector at the first focus of the ellipsoid; the detector was then moved to the second focus of the ellipsoid and the specimen was mounted at the first focus, and the reflected flux was measured. The ratio of the reflected flux to incident flux, corrected for mirror losses, was taken as the absolute spectral reflectance of the specimen at the wavelength of the incident flux.

\section{2b Golay-cell Detector Problems}

A large-area Golay-cell detector was used in the early work. Its sensitive area was one centimeter in diameter. When used with the sensitive diaphragm in a horizontal position the detector proved to be highly microphonic. Elaborate precautions were taken to isolate the detector from stmucture-borne noise by mounting it on a seismic table which was separated frcm the rest of the equipment, and to isolate it from air-borne noise by enclosing the entire optical path from the exit port of the monochromator to the detector in acoustical insulation. These precautions reduced the noise to a point where useful measurements could be made.

Measurements with this early design of the reflectance of both flowers of sulfur and of a rhodium mirror gave values of reflectance as low as 0.50 . The reflectance of these materials at the wavelengths at which measurements were made was known to be greater than 0.90 . Mirror losses were computed to be appreciably less than 0.05 in the case of sulfur and 0.03 in the case of the rhodium mirror. Obviously the measured reflectances were grossly in error.

One of the objectives of the work with the instrument was to measure the reflectance of specimens at temperatures above and below room temperature. A specimen heater, capable of operation up to $800^{\circ} \mathrm{K}$, was designed and built. When a test was made of a heated specimen, the Golay-cell detector failed because of the background radiation when the specimen reached a temperature of about $350^{\circ} \mathrm{K}$. 
Because of these difficulties encountered with the Golay-cell detector, the decision was reached that a different type of detector was needed.

\subsection{Current Work}

\section{3a Detector Developments}

\section{3a-1 Thermopile Detectors}

Two custom-built thermopile detectors with a viewing area of one square centimeter were obtained and tested. Tests of signal-to-noise ratio, sensitivity and linearity in the 1 to 15 micron wavelength range indicated that they should be satisfactory for the intended purpose

A preliminary measurement of the reflectance of flowers of sulfur at $5 \mu$ gave an uncorrected reflectance of about 0.50 , which is obviously in error. It was thought that some of the losses might be caused by aberrations of the ellipsoidal mirror, which resulted in some of the flux reflected from the specimen being focused outside the sensitive area of the detector. Photographs were taken of (1) the beam incident on the specimen, and (2) the beam incident on the detector after reflection from the specimen. By careful adjustment of the position of the specimen and detector relative to the ellipsoidal mirror, it was possible to obtain an image at the detector of the illuminated spot on the specimen that fell entirely within the sensitive area on the detector. However, this careful adjustment did not appreciably increase the measured (uncorrected) reflectance of flowers of sulfur at 50 .

\section{3a-2 Spatial Sensitivity of Detectors}

Further investigation revealed that both the Golay and thermopile detectors gave varied response over their sensitive areas. Quantitative tests were made to show the magnitude of this effect.

The Golay-cell detector was mounted, with its sensitive area in a vertical plane, on an automatically-driven micrometer head that could move it horizontally in the plane of the sensitive area at a rate of about $0.08 "$ per minute. A stationary aperture stop, having a circular opening $1 / 16 "$ in diameter, was mounted directly in front of the detector. The detector was illuminated, through the aperture stop, by filtered radiation from the Globar source, chopped at 10 cycles per second. Three different filter combirations were used, to give two relatively narrow bands centered at about 2 and 9 microns respectively, and one covering the range of 15 to 20 microns.

Two scans across the sensitive area of the cell were made with radiation passing each of the filter combinations, along two diameters of the cell nomal to each other, designated $A-A^{\prime}$ and $B-B^{\prime}$ respectively. Results of the scans are shown in Figures 24 and 25 . Each curve represents the relative response of the detector-amplifier combination to the radiant energy passing through the aperture, as a function of the distance along the scan. Different gain settings were used for the different spectral regions, hence the different curves are not directly comparable.

The curves in Figure 24 show that along the diameter A-A' an area of highest sensitivity exists 2-3 mm from the right side of the sensitive area, and a second, much lower peak in sensitivity occurs near the center. These results were not expected, and there appears to be nothing in the basic design of the detector to account for them. Apparently the absorptance of the absorbing membrane varies over its surface. The curves in Figure 25 show that along the diameter $B-B^{\prime}$ the sensitivity is symmetrical, with two peaks each about $3 \mathrm{~mm}$ from the edge of the sensitive area, with slightly less sensitivity at the center. There is a shortrange periodicity, with a minor peak about every millimeter. The curves in Figure 25 are more nearly what would be expected from the basic design of the cell.

Similar measurements were made of the variation in sensitivity over the sensitive area of the new thermopile detector that was referred to above. In this case the Globar source was replaced by a tungsten filament lamp, and no filters were used. A diffusing screen was placed immediately in front of the detector for some of the scans. The diffusing screen was a sodium chloride window that had been depolished on the side away from the detector. Diffusing screen no. 2 was $5 \mathrm{~mm}$ thick, and one surface had been ground with $9.5 \mu$ abrasive. Screen no. 3 was $2.5 \mathrm{~mm}$ thick, and one surface had been ground with $50 \mu$ abrasive.

The thermopile consists of ten plates, each approximately $2 \times 5 \mathrm{~mm}$ in size, arranged in two columns of five rows each to form a sensitive area one centimeter square. A thermocouple was attached to the back of each plate, and the 10 thermocouples were connected in series 
to form the thermopile. The scans in the $A-A^{\prime}$ direction were made across the center of the sensitive area along the line between the two columns. The scans in the $B-B^{\prime}$ direction were made across the center of the sensitive area, along the long axis of the two plates in the third row. In both cases scans were made (1) using incident radiation chopped at 13 cycles per second and an amplifier tuned to 13 c.p.s. and (2) unchopped incident radiation and a DC amplifier. Results are shown in Figure 26 for the AC scans, and in Figure 27 for the DC scans.

In Figure 26 for the $A-A^{\prime}$ scans it can be seen that, with the screen holder only, there are three distinct peaks, with some indication of two others, corresponding to the position of the five rows of plates. Diffusing screen no. 2 was quite effective, and gave relatively uniform response across the sensitive area. In the B-B' scan without the screen holder, it can be seen that the plate on the right had greater sensitivity than that on the left. The screen holder alone had very little effect, as would be expected. The no. 2 diffusing screen has practically eliminated the short-range peaks seen in the other two scans, but the greater sensitivity of the plate on the right is still evident.

In Figure 27, the DC scans in the B-B' direction are somewhat similar to the equivalent AC scans for the screen holder only and diffusing screen no. 2 , but with no screen holder the curve is relatively smooth, and shows that the plate on the left has greater sensitivity than that on the right. The scans in the $A-A^{\prime}$ direction show five distinct peaks with no screen holder. The curve with the screen holder only is qualitatively similar to the equivalent $\mathrm{AC}$ scan, and relatively uniform response is obtained with the no. 2 diffusing screen.

The variations in sensitivity over the sensitive area of the detectors could readily account for the unsatisfactory results reported previously for the ellipsoidal mirror reflectometer. The incident beam is concentrated in an area on the specimen approximately $2 \mathrm{rm}$ square, while the radiant energy reflected from the specimen is focused into an image approximately $1 \mathrm{~cm}$ square.

\section{3a-3 Angular Sensitivity of Detectors}

Since the detector needed to be equally sensitive to radiation striking at any angle from 0 to $24^{\circ}$, the angular sensitivity of the thermopile detector was measured. To make this measurement, the detector was mounted on a milling head with its sensitive area in a vertical plane and with the two columns of plates vertical, in a position such that the center line of the sensitive area coincided with the vertical axis of rotation of the milling head. An image of the exit slit of the monocnromator, $3 \times 3 \mathrm{~mm}$ in size, was focused on the center of the sensitive area from a direction nomal to it, by means of a 6-in. diameter spherical mirror having a 49-in. radius of curvature. The cone of rays thus had a half-angle width of about $3 \frac{10}{2} \circ$. The monochromator was adjusted to give a band of flux centered at 2.2 microns. The response of the detector was recorded as $R_{n}$ when the axial ray of the incident beam was normal to the sensitive area. The milling head was then rotated to give incident angles of $5,10,15,20,25,30,40,50,60$, and $70^{\circ}$ to the normal, and the response of the detector was recorded at each setting as $R_{\theta}, \theta$ being the angle of incidence. The data were normalized by dividing each reading by the reading at normal incidence, and plotted as a function of angle of incidence to produce the curve shown in Figure 28. Similar measurements were made with a cover plate $0.15 \mathrm{in}$. in thickness with a $1 \mathrm{x} 1 \mathrm{~cm}$ hole, centered over the sensitive area. The entire procedure was then repeated with flux of 8 microns wavelength.

The experimental curves are compared in Figure 28 with two computed theoretical curves. The top curve, in wich $R_{n} / R_{\theta}=1$ at all angles would be obtained if the detector was equall sensitive to flux striking it at all angles, and if all of the incident flux struck the sensitive area. The lower curve, in which $R_{n} / R_{\theta}=\cos \theta$, would be obtained if the detector was equally sensitive to flux striking it at all angles, and was completely filled at normal incidence.

From the experimental curves in Figure 28 it can be seen that the sensitivity increases slightly from normal to $20^{\circ}$, then decreases. The increase is undoubtedly due to the fact that as the illuminated area of the detector increases, the more sensitive areas, as shown in the previous section, become illuminated. The sharp drop in response beginning at about $30^{\circ}$ is due to some of the flux being lost, either not admitted through the window or not striking the sensitive area if admitted. The presence of the cover plate increases the rate of fall off in this range, as might be expected. 


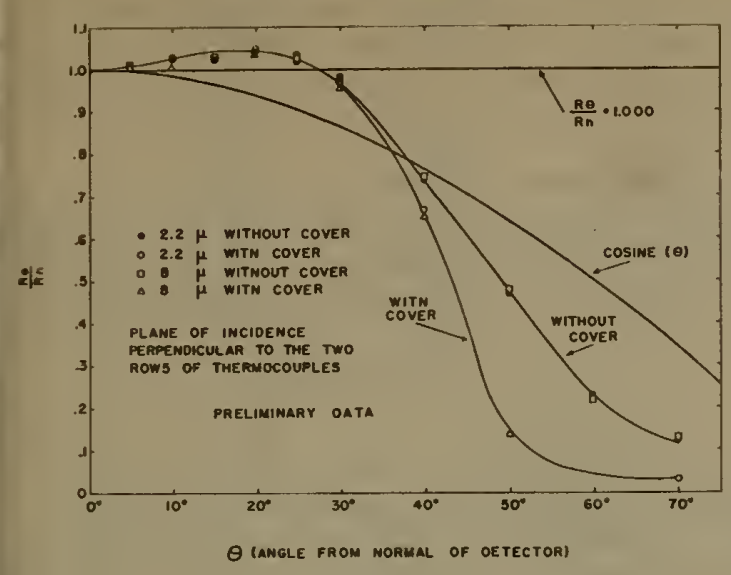

Figure 28. Angular Sensitivity of the Thermopile Detector in the $A-A^{\prime}$ Plane at 2.2 and 8.0 Microns. The curves show the variation in response to a beam of constant flux, $3 \mathrm{~mm}$ square, as the angle of incidence is varied in the $A-A^{\prime}$ plane.

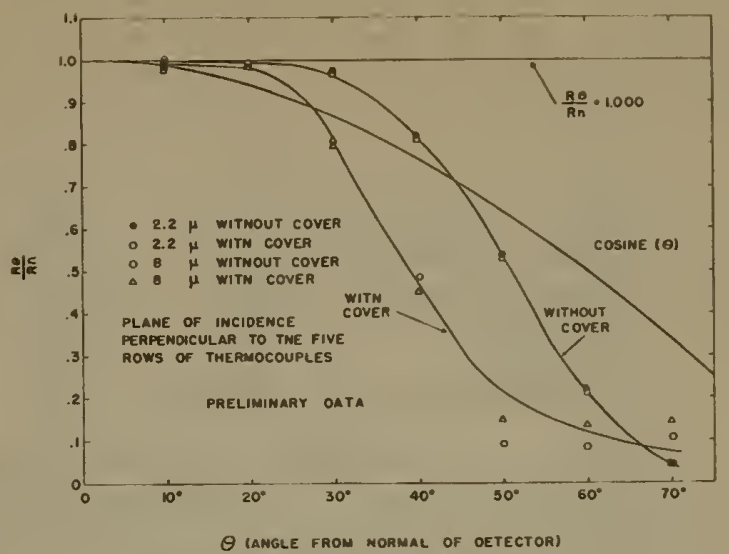

Figure 29. Angular Sensitivity of the Thermopile Detector in the B-B' Plane at 2.2 and 8.0 Microns. The curves show the variation in response to a beam of constant flux, $3 \mathrm{~mm}$ square, as the angle of incidence is varied in the $B-B^{\prime}$ plane.

\section{DIFFUSING ELBOW}

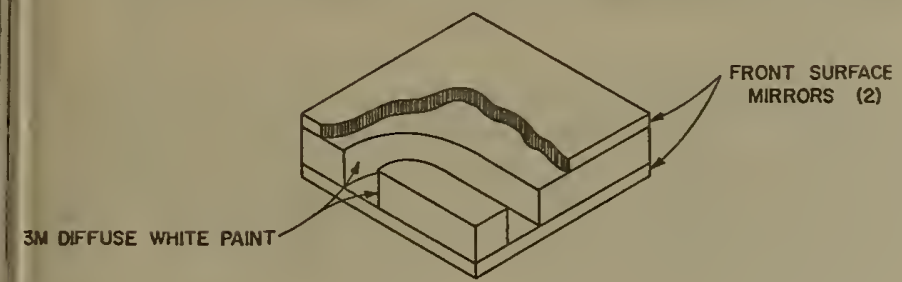

Figure 30. Diffusing Elbow Designed to Reduce Areal Sensitivity of Thermopile Detector.

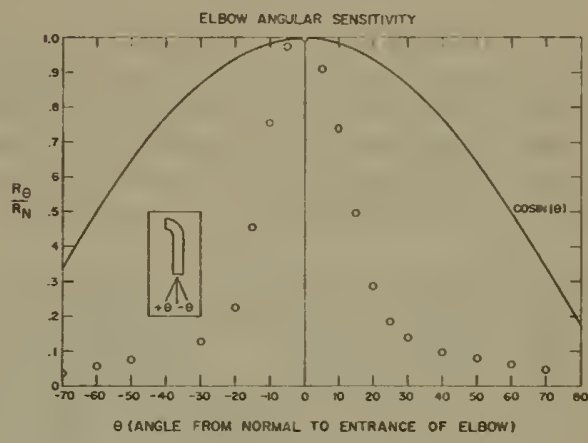

Figure 31. Angular Sensitivity of Thermopile Detector with Diffusing Elbow, in the Plane Perpendicular to the Diffusing Surfaces. 
Similar tests were made with the detector mounted with the five rows of plates vertical. The results shown in Figure 29 are simllar to those in Figure 28 except that the increase in signal from 0 to $20^{\circ}$ was not observed. This is undoubtedly due to the fact that as the angle was increased, more plates were illuminated, but in the same relative areas, hence the signal remained constant.

The curves plotted in Figures 28 and 29 show no significant differences for flux of 2.2 and 8 microns, respectively. The fact that the curves are nearly 1 lat from $0^{\prime}$ to $25^{\circ}$ indicate that variation in angular sensitivity will be no problem with the ellipsoidal mirror reflectometer since the marginal rays strike the thermopile detector at an angle of approximately $24^{\circ}$ from the normal.

\section{3a-4. Flux Averaging Devices}

The data on the variation in spatial sensitivity of the detectors referred to in Section $3.3 a-2$ indicate that a flux-averaging device will be required for use with any of the available large-area detectors. The function of such a device is to spread the available flux, regardless of image size, shape or intensity distribution, uniformly over the sensitive area of the detector.

The literature provides at least two references to this problem. The first is the work of Bennett and Koehler [5], who used a small integrating sphere to average out the signal over a photomultiplier detector. The second is the work of Rohzhin [6], who tried light ducts and integrating spheres to average the signal over the sensitive area of a photomultiplier. However, these references offer solutions only in the ultraviolet and visible protions of the spectrum, where good integrating sphere coatings are available. In the infrared, the spectral region for which the reflectometer was specifically designed, no one has yet shown that satisfactory integrating sphere coatings exist for use beyond about 4 microns. However, Birkebak [7] has shown that sulfur is both a good diffuser and reflector at 2 microns and at 4 microns, and he assumes that it is usable out to 10 microns. However, he does not mention the specific form of sulfur that was used for his measurements, or his method of applying it to the sphere wall. Kronstein et al [8] report that mu sulfur is a good reflector out to 15 microns, and give a spectral reflectance curve. They did not, however, use it as a sphere coating.

Any averaging device reduces the efficiency of a detector system, because some of the incident flux is absorbed in the diffuser, and some is scattered away from the sensitive area of the detector and is lost. Since the flux avialable for measurement at wavelengths near 1 and 15 microns is already very low, it is important that losses in the diffuser be kept as low as possible. In general, the losses in the diffuser tend to increase with an increase in its efficiency as a diffuser.

Three different types of diffusing devices were investigated. They are listed in estimated order of increasing efficiency of diffusion as: (1) a diffusing screen placed directly over the detector, (2) a light duct with diffusing walls or a diffusing surface in the system, and (3) an integrating sphere coated with a material having high reflectance in the infrared and sufficient diffusing power to permit it to be used as an averaging device.

The first device tested was a roughened sodium chloride window. The data in Figures 26 and 27 indicate that it was only partially effective. Another diffusing screen, which has not yet been tested, can be made by sandwiching a thin transmitting layer of small particles of any convenient transparent material between two polished windows of sodium chloride. Sodium chloride, mu sulfur, and cesium bromide would be suitable for such use.

The second effort was to construct a diffusing elbow, shown in Figure 30 . This elbow essentially eliminated the spatial. sensitivity when the flux was incident on one end of the elbow, and the detector was placed at the other end. However, Figure 31 indicates that the sensitivity of the elbow-detector combination varied with angle of incidence across the diffusing surfaces, while Figure 32 shows no such angular sensitivity in the plane of incidence across the mirror surface. Figure 33 is yet another design, in which the walls are all mirrors, except for the diffuse $45^{\circ}$ surface used to reflect the incoming radiation toward the detector. One diffusely reflecting surface for use with this device consists of a series of spherical depressions in aluminum, each of $1 / 16$ in. radius, spaced 0.088 in. apart in a hex- 

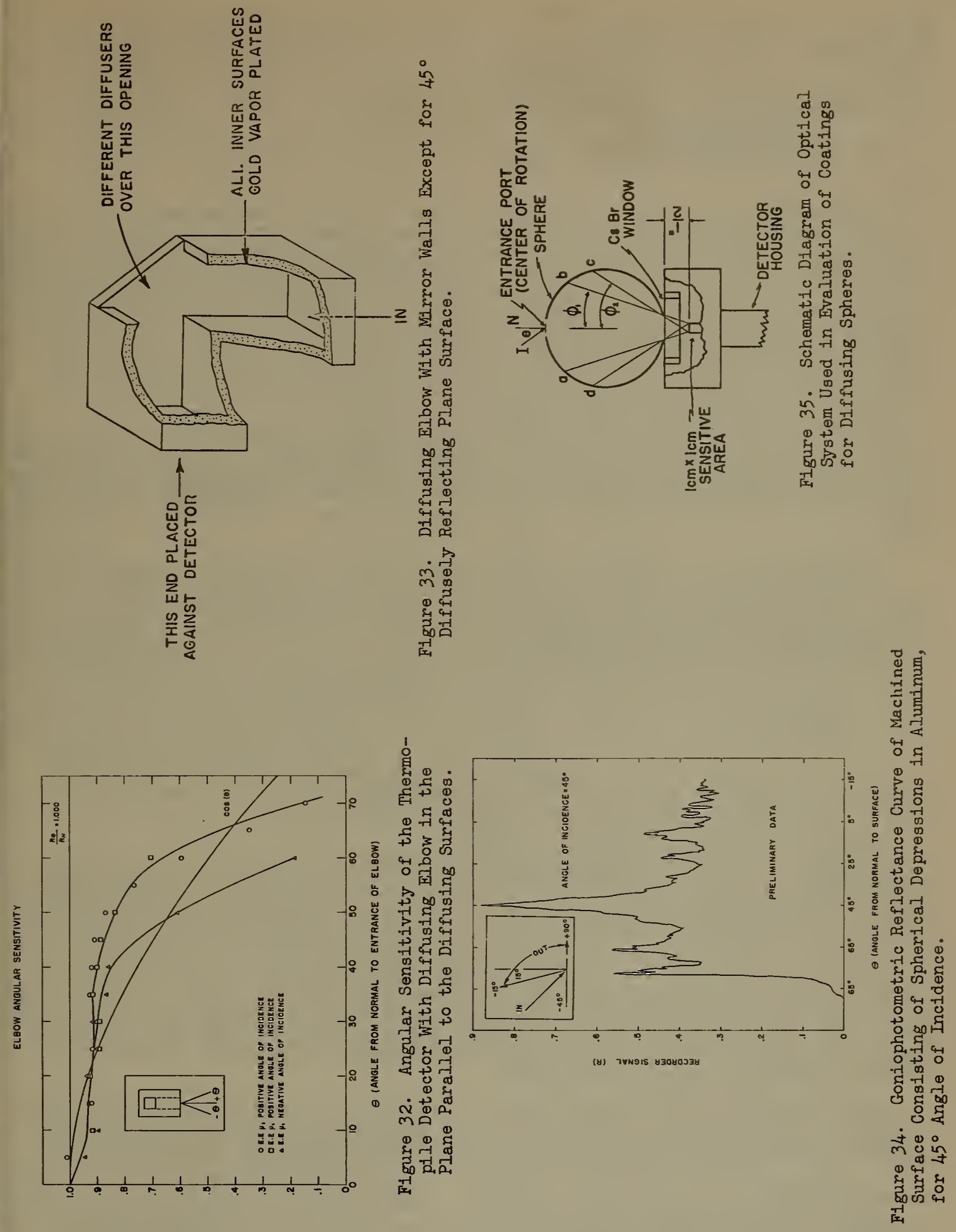
agonal close pack array. Goniophotometric reflectance curves for this surface for white light incident at $45^{\circ}$ are shown in Figure 34. This surfice has since been liquid honed, and then gold plated. The liquid honing gives a diffusing surface of small roughness, which, in.combination with the large roughness of the spherical depressions, should reduce the height of the specular peak at $45^{\circ}$ seen in Figure 34 . Other diffusing surfaces to be tested in this elbow include mu sulfur, and a gold-plated wire mesh manufactured by the General Electric Co.

The final approach was that of developing a small conventional integratirig sphere that would be suitable for use throughout the 1 to 15 micron wavelength range.

As preliminary trial, a small integrating sphere diffuser, $4.5 \mathrm{~cm}$ in diameter, coated on the inside with $3 \mathrm{M}$ white Velvet coating*, was designed and built. The entrance aperture and viewing port were located $90^{\circ}$ apart on a great circle. Since the detector plus diffuser would not fit into the available space at the first focus of the ellipsoid, it was not possible to make direct measurements of the incident flux. However, comparison reflectance measurements of several specimens having different geometric distributions of reflected flux gave results that were in reasonable agreement with those obtained with other instruments. The 3M coating is usable only at wavelengths below about 24 , but these preliminary measurements indicated that the basic design of the instrument was sound, and that it should be capable of giving accurate results if a suitable infrared diffuser could be found.

Three tests were devised to evaluate the effectiveness of sphere coatings. The general optical system for these tests is shown schematically in Figure 35. A $152 \mathrm{~mm}$ ( 6 in.) diameter spherical mirror of $1.24 \mathrm{~m}$ ( $49 \mathrm{in}$.) radius is used to focus the beam from the exit slit of the monochromator onto the entrance port of the sphere. The sphere is mounted in a milling head, so that it can be moved $20 \mathrm{~cm}$ ( $8 \mathrm{in.}$ ) in the $x$ and $y$ directions, and rotated $360^{\circ}$ in the $x-y$ plane.

In the first test, the sphere was mounted with its entrance port at the center of rotation of the milling head, and the incident beam was centered on the entrance port. The sphere was then rotated, and the response of the detector was recorded as a function of the angular position of the sphere, measured as the angle between the axial ray of the incident beam and the normal to the sphere entrance port. In the second test, the sphere was mounted as before, but in this case it was moved along the axial ray of the incident beam, and the detector response was recorded as a function of the sphere position. In the third test a modified sphere was used, in which the entire illuminated area of the sphere wall was viewed in one configuration.

Data from the first test were normalized by dividing the signal from the detector at each angle of view by the signal obtained with the axial ray normal to the entrance port ( $0{ }^{\circ}$ angle of view). Typical data are shown in Figures 36 through 40.

If a perfect integrating sphere were tested in this way, and the detector viewed only a portion of the sphere wall, as illustrated in Figure 41, then the signal from the detector would change as the directly illuminated spot moves around the sphere, by an amount proportional to the difference in brightness between the areas of the sphere wall that are directly illuminated and those not directly illuminated. If the illuminated area is not viewed by the detector, none of the flux will be received by the detector on the first reflection. Thus, for a surface that approaches an ideal integrating sphere coating, the curve of response as a function of angle should show two ranges of constant response with a smooth monatonic transition. One range would represent those angles at which the detector wiews none of the illuminated area, and the other would represent those angles at which it views the entire illuminated area, and the transition would represent those angles at which the detector views an increasing fraction of the illuminated area.

Jacques and Kuppenheim [1] show that for high efficiency, a high reflectance, diffusely reflecting sphere wall and a small sphere diameter are required. High sphere efficiency is required in this application, because the amount of flux available for measurement is near

* Available from 3M Reflective Products Div., Minn. Mining \& Mfg. Co., 2501 Hudson Rd., St. Paul 19, Minn. Goniophotometric measurements made at NBS show that this coating closely approaches a cosine diffuser. 


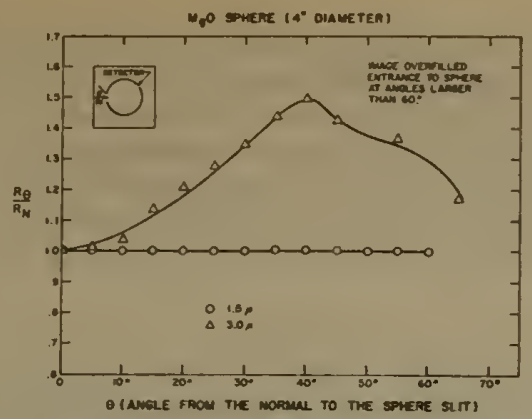

Figure 36. Angular Sensitivity of Thermopile Detector with 4-Inch Diameter MgO Coated Sphere, at 1.5 and 3.0 Microns.

GOLO PLATE COPPER SPHERE $\left(4^{\circ}\right.$ DAAMETER)

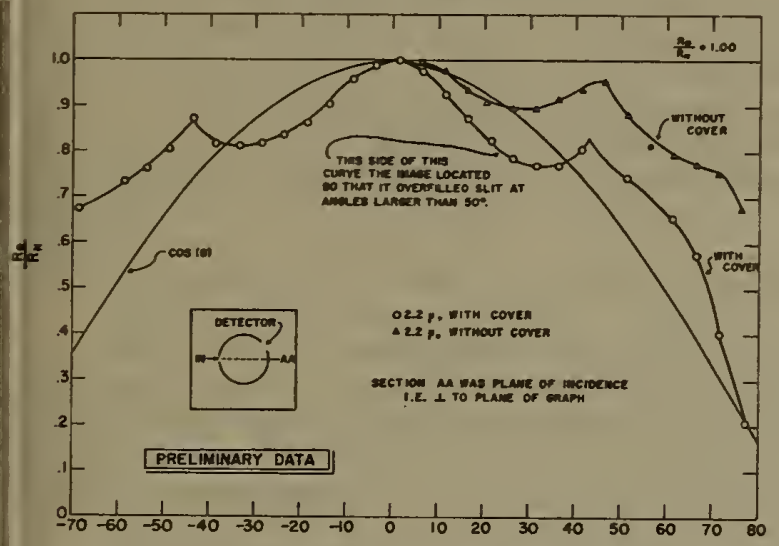

$\theta$ CANGLE FROM THE NORMAL OF SPHERE SLIT?

Figure 38. Angular Sensitivity of Thermopile Detector with 2-Inch Diameter I-Shot Gold-Plated Sphere at 2.2 Microns.

$2^{*}$ DIA SULFUR SPMERES

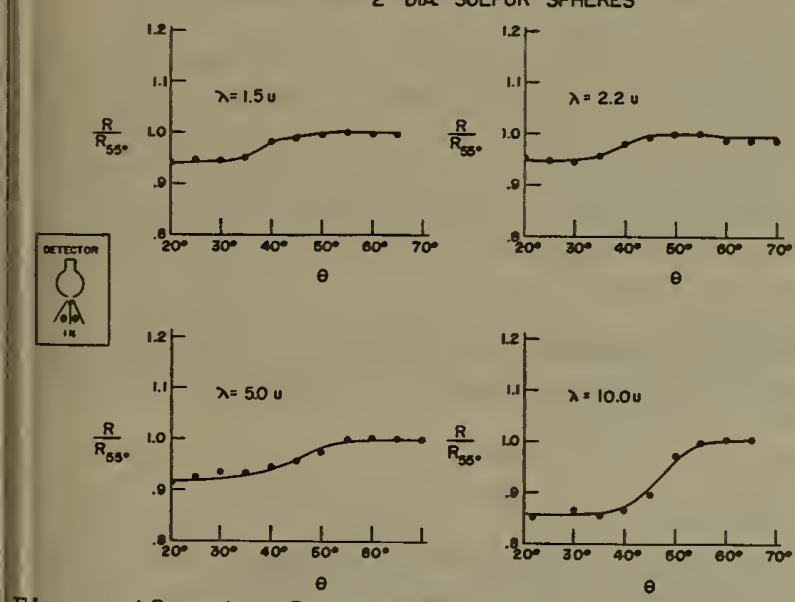

Figure 40. Angular Sensitivity of Thermopile Detector with 2-Inch Diameter Sphere Coated with Hand-Peened mu Sulfur, at 1.5, 2.2, 5.0 and 10.0 Microns.
L SHOT GOLD PLATED SPHERE (2" DIAMETER)

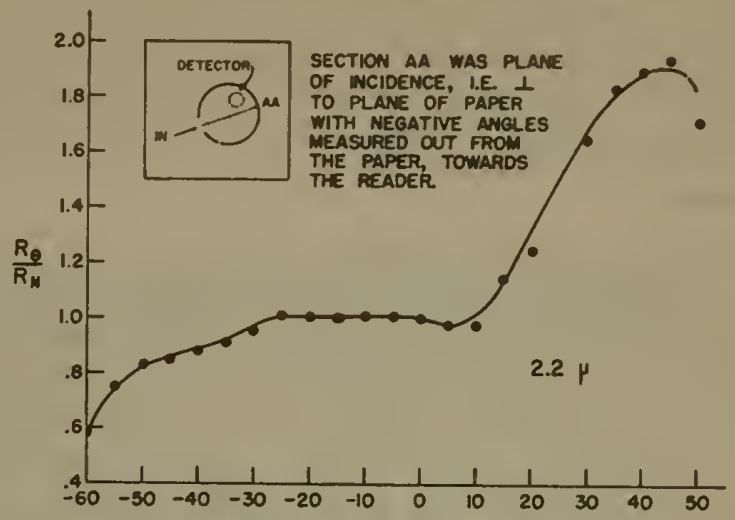

OCANGLE FROM THE NORMAL TO SPHERE ENTRANCE)

Figure 37. Angular Sensitivity of Thermopile Detector with 4-Inch Diameter I-Shot GoldPlated Sphere at 2.2 Microns.

S-460 SHOT GOLO PLATED SPMERE (2" DIAMETER)

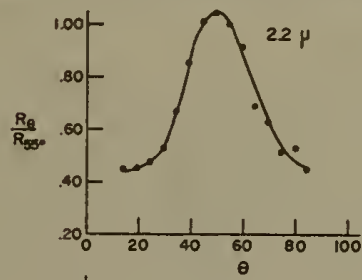

O IS ANGE MEASUREO FROM
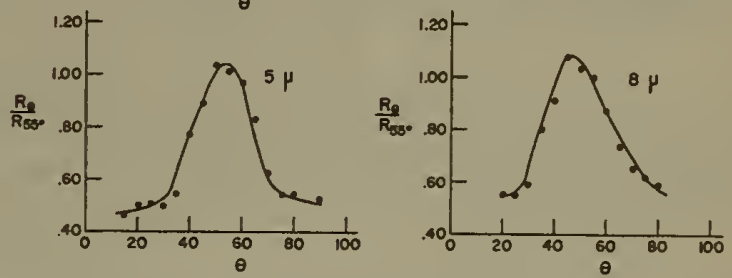

Figure 39. Angular Sensitivity of Thermopile Detector with 2-Inch Diameter S-460 Shot Gold-Plated Sphere at 2.2, 5 and 8 Microns.

$R_{0}$ IS READING FOR IMAGE FOCUSED ON THE ENTRANCE TO THE

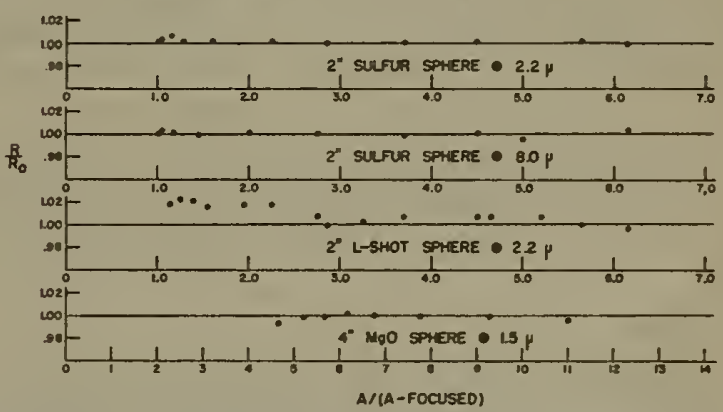

Figure 4l. Response Ratio of Thermopile Detector with Indicated Spheres, Plotted as a Function of the Ratio of the Illumtnsted Areas on the Sphere Wall, for Beams of Constant Flux. 
the lower limit of the useful range of the detector, particularly at the longer and shorter wavelengths, near 1 and 15 microns. Polished metals have high reflectance in the infrared, but are not good diffusers. Roughening the surface of a metal decreases reflectance, but increases diffuseness. It may be possible to produce a usable sphere coating by suitable contouring of a metal surface, or by applying a vacuum-deposited metal over a contoured substrate. Birkebak [7] showed sulfur to be diffuse at 2 and 4 microns, and Agnew and McQuistan [9] showed it to be diffuse to the flux from a Globar at wavelengths above and below 4 microns.

A number of different spheres were built and coated. These included 50mm (2-in.) diameter spheres with gold plated I-glass shot* (1) and S-430 shot* (2) surfaces, and handpressed mu sulfur (3) coatings, and 100mm (4-in.) diameter spheres with gold plated I-shot surface (4) and $\mathrm{MgO}$ coating (5). These spheres were evaluated at several different wavelengths, and with different detector configurations.

The data for the $100 \mathrm{~mm}$ (4-in.) diameter MgO coated sphere are shown in Figure 36 . At $1.5 \mu$, where $\mathrm{MgO}$ is a good sphere coating, the signal was constant to within $1 \%$ with angle out to an angle of $60^{\circ}$, and then it decreased due to image spill-over from the sphere entrance port. At $3.0 \mu$, where the reflectance of $\mathrm{MgO}$ is low and there is a significant specular component, the signal varied appreciable with angle. (It should be noted that with the detector configuration used in these tests, the detector viewed at least a small portion of the illuminated area of the sphere at all angles.) Thus, the curves in Figure 36 show a marked difference in the behavior of $\mathrm{MgO}$ at a wavelength where it is known to be a good sphere coating, and at a second wavelength where it is known to be a poor integrating sphere coating.

Figures 37, 38, and 39 show data for the gold-plated spheres. Figure 37 is for the $100 \mathrm{~mm}$ (4-in.) diameter I-shot sphere at $2.2 \mu$, and indicates that this combination is not useful as an integrating sphere at this wavelength. In this case the detector was in the plane of incidence. Figure 38 is for a $50 \mathrm{~mm}$ (2-in.) diameter sphere with a similar coating. In this case, the detector is centered on a radius of the sphere that was inclined about $20^{\circ}$ above the plane of incidence, so that the illuminated area was directly below the detector at an incident angle of about $-15^{\circ}$. From $10^{\circ}$ to $50^{\circ}$ the respones varies markedly with angle of incidence, indicating that the sphere wall is specular, and hence not suitable for an integrating sphere. From $-25^{\circ}$ to $0^{\circ}$, there is little variation in respones with angle of incidence. At these angles of incidence the illumiated area is near and just below the detector port, where it is not viewed by the detector. Apparently, if the incident flux can be confined to this area of the sphere, it might be suitable for use as a diffuser at $2.2 \mu$, if not as an integrating sphere. It remains to be determined whether the curve will be similar at longer wavelengths, since the roughness of the surface is on the order of $30 \mu-$ in. rms, and the geometric distribution of reflected energy might change with wavelength.

Figure 39 shows results obtained with the gold-plated S-460 shot 50mm (2-in.) diameter sphere, at wavelengths of 2.2, 5, and 8 microns. In this case the detector port is in the plane of incidence, and is diametrically opposite the entrance port. The curves indicate that there is a large specular component to the reflected flux, at $45^{\circ}$, and that the reflectance characteristics do not change appreciably with wavelength. The $S-460$ shot surface has a roughness of about $150 \mu-i n$. rms, hence no effect of wavelength would be expected in this range.

From the tests reported above, it is concluded that the gold plating on a roughened substrate does not appear promising as a true integrating sphere coating, but it appears likely that suitable diffusers can be designed for use with such surfaces.

* I-shot spheres are available from the Pangborn Co. They are glass spheres, $-200+325$ mesh SAE. A surface blasted with these spheres has a roughness of about $30 \mu-$ in. rms. The S-460 shot is also available from the Pangborn Co. These are steel spheres, 10-mesh SAE, and produce a surface of about $150 \mu-i n$. rms roughness. 
Figure 40 shows data obtained on the 50 mm (2-in.) diameter sphere coated with hand-peened mu sulfur.* These data show that the sulfur coating is highly diffuse at 1.5, 2.2, 5.0, and

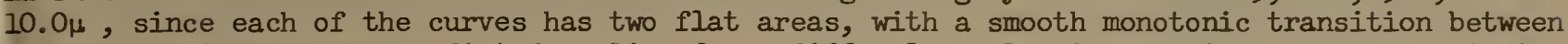
them. This is what was predicted earlier for a diffusely reflecting coating. The tests indicate that a sulfur coated sphere would be a satisfactory diffuser if the sphere were used in a configuration such that the illuminated area was either always totally viewed or never viewed by the detector.

A second series of tests was performed in an effort to establish more directly the suitability of the various coated spheres for use with the ellipsoidal mirror reflectometer. In these tests the detector port and entrance port were centered on radii of the sphere $135^{\circ}$ apart, as indicated in Figure 35. The sphere was mounted in the milling heat (Figure 35) with the axial ray of the incident beam centered on and normal to the entrance port. The sphere was moved along the axial ray of the incident beam, and the detector response was recorded as a function of sphere position. This test was designed to evaluate the variation in detection response with size of the illuminated area on the sphere wall, when the total incident flux is held constant. Since the incident beam is diverging from a focus, the size of the illuminated area could be varied from the minimum size when the beam was focused on the sphere wall [ or inside the sphere for the $100 \mathrm{~mm}$ (4-in.) diameter sphere] to a maximum size when the marginal rays fell just inside the entrance port. The maximum size ratio was 6.15 to 1 for the $50 \mathrm{~mm}$ (2-in.) diameter sphere and about 2.36 to 1 for the $100 \mathrm{~mm}$ (4-in.) diameter sphere. The measured sphere position was converted to the area of sphere wall illuminated by the incident beam from the beam geometry, and each area was divided by the cross-sectional area of the beam at the focal point to obtain the area ratio for each position. The detector response $Q$ at each position was divided by the response $Q_{0}$ at the position where the beam was focused on the entrance port, to obtain the response ratio $Q / Q_{0}$. Response ratio was then plotted as a function of area ratio to obtain the curves shown in figure 41.

The foregoing test was designed to simulate the conditions that exist when the detector is moved from the first to the second focal point of the ellipsoidal mirror. At the first focal point, the illuminated area on the sphere wall will be on the order of $10 \mathrm{x} 12 \mathrm{~mm}$, and at the second focal point it will be on the order of $18 \times 20 \mathrm{~mm}$, hence the test conditions fully cover the range of areas that will be encountered in service.

The data in Figure 41 show that with the $100 \mathrm{~mm}$ (4-in.) diameter MgO sphere at 1.5k, there was a spread in response ratio of about 0.8 percent. These results are consistent with data in Figure 13, which show a spread in response of about one percent with change in angle of incidence. The spread in response in Figure 41 is in part due to the fact that the detector viewed part of the illuminated area at some settings.

The $50 \mathrm{~mm}$ (2-in.) diameter I-shot sphere at $2.2 \mu$ showed a spread in response of slightly more than 2 percent, which is above the acceptable limit. However, it is felt that with careful placement of the illuminated area and internal baffling of the sphere, this spread can be reduced to below one percent, so that the gold-plated I-shot surface can be used in a diffuser, particularly at wavelengths where the reflectance of sulfur is low.

The $50 \mathrm{~mm}$ (2-in.) diameter sulfur-coated sphere showed only a 0.5 percent spread in response at 2.2 and $8.0 \mu$. Thus, the sulfur-coated sphere should be satisfactory as a diffuser out to at least $8 \mu$. Because of the lower reflectance of sulfur at longer wavelengths, its use beyond $10.5 \mu$ is questionable. The net results of these tests is to show that the sulfurcoated sphere is an acceptable averaging device in the $2-8 \mu$ range, and that the I-shot goldplated sphere may, with careful design, be used in the $10-15 \mu$ range.

The sulfur-coated spheres that were tested had been coated by pressing the sulfur in place by hand. A new technique has since been developed for application of the sulfur by spraying as an alcohol suspension. It is believed that the spheres coated by the new techniques have a more nearly uniform coating than those prepared by hand pressing, and, hence, may show even smaller spreads in response with variation in size of the illuminated area.

* Crystex 90 brand sulfur, purchased from the Stauffer Chemical Co. This is $90 \%$ insoluble (mu) sulfur, $99.5 \%$ elemental sulfur, with 3-4 $\mathrm{k}$ particle size. Data on the reflectance of sulfur are given in reference 8. 


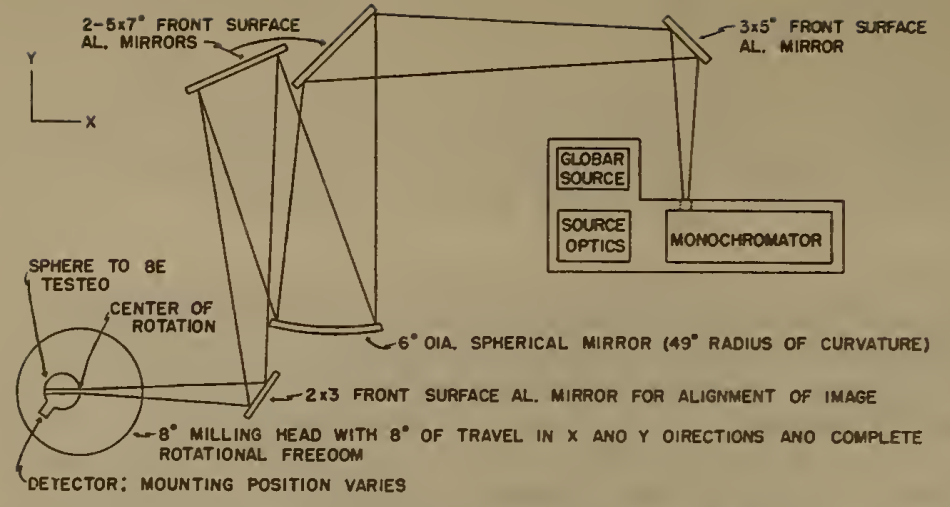

SPATIAL AND ANGULAR SENSITIVITY TEST APPARATUS

Figure 42. Detector and Beam Conflgurations for a Test of Efficiency of a Material as an Integrating Sphere Coating.

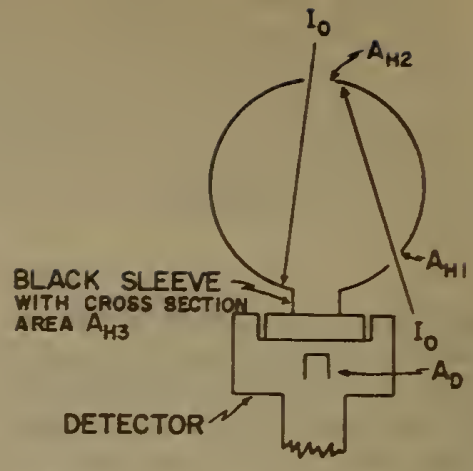

Pigure 43. Theoret1cal Model for Developing Theory of Tests of Integrating Sphere Coatings.

The tests described above indicate qualitatively that sulfur may also be useful as an integrating sphere coating. With this in mind, \& third test to quantitatively eveluate integrating sphere coatings was devised. The results of the first test, shown in Figure 40 for sulfur, in which the illuminated area was moved around a great circle of the sphere, gave a qualitative indication of the ut1lity of a surface in an integrating sphere. However, the values for the difference in readings when the illuminated area was not viewed directly by the detector (ares c-d in F1gure 11, low flat portion of the curves in F1gure 40), and when 1t was viewed directly by the detector (area $a-b$ in Flgure II, hIgh Ilat portion of the curves in F1gure 40), were lower by a factor of as much as 5 than would be predicted by theory. Apparently, the experimental set-up did not pit the theoretical model. The flux from the 1 Iluminated area not directly vlewed by the detector (area $c-d$ in FIgure II) could reach the detector by paths other than by being mult1ply reflected from the d-a-b-c area viewed by the detector, by ( 8 ) hitting the $11 p$ of the detector port and being reflected to the detector, and (b) being diffused to the detector by scratches on the CsBr window. The net effect would be to incresse the helght of the low flat portion of the curves in Figure 40. The 1llumination in the a-b ares of Flgure 35 is incident at large angles of Incldence, where even the best diffusers tend to become somewhat specular. Thus, the Plux tends to be specularly reflected around the sphere wall into the area b-a to which the detector is less sensitive, instead of being diffusely reflected to the detector. The net effect would be to reduce the helght of the high flat portion of the curves in Flgure 40. These two effects undoubtedly are responsible for the low ratio of the two $s$ ignals, as compared to the theoretical model.

Detector and beam conflgurations for a new test are given in Flgure 42 . In this test both beams are incldent from directions near normal, and flux from areas not vlewed by the detector can no longer reach $1 t$ by indirect paths. Spheres of this configuration are now being prepared for test.

\section{3b. Theory for Tests of Integrating Sphere Coatings.}

Figure 43 1llustrates the theoretical model to be studied. The flux from the first reflection $w 171$ be considered separately from that due to multiple reflections in the sphere. To get a quantitative value for the ratio of the two flat rogions discussed above, it 18 necessary to account for the flux that gets to the detector in each case.

Case 1. The detector does not vlew the first rellection of the incldent flux ( $\left.I_{0}\right)$. Then the flux reflected by the phere wall on the first reflection is

$$
R_{S}=I_{0} \rho_{S}
$$


and none of this reflected flux implnges directly on the detector. This flux is then reflected by the entire spere (except for the portion lost out the entrance and extt ports). The flux reflected twlce by the sphere wall

$$
R_{1}^{\prime}=I_{0} \rho_{S}^{3} \frac{A_{S H}}{A_{S}}
$$

Where $A_{S}$ is the total area of the phere $\left(A_{S}=4 \pi R^{2}\right)$ and $A_{S H}=A_{S}-A_{H 1}-A_{H 2}\left(A_{H 1}\right.$ and $A_{H 2}$ are the areas of the extt and entrance ports, respectively). Of the flux $R_{1}^{\prime}$, only $R_{2}^{\prime}$ reaches the detector sensing element,

$$
R_{2}^{\prime}=I_{0} \rho_{S}^{2} \frac{A_{S H}}{A_{S}}\left[\frac{A_{D V}-A_{H 2}}{A_{S}}\right] f_{1}
$$

where ADV is the area of the shere fully viewed by the detector, (ADV - A H2)/AS is the portion of the twice reflected flux in the area viewed by the detector, and $P_{1}$ is the diffuse configuration factor from (ADV - AH2) to the sensing element of the detector.

Similarly, on the third reflection the amount of flux reaching the detector 1 ,

$$
R_{3}^{\prime}=I_{0} \rho_{S}^{3}\left[\frac{A_{S H}}{A_{S}}\right]^{3} \frac{A_{D V}-A_{H 2}}{A_{S}} f_{1}
$$

and on the $n^{\prime}$ th reflection, it is,

$$
R_{n}^{\prime}=I_{0} \rho_{S}^{n}\left[\left.\frac{{ }_{S H}}{A_{S}}\right|^{n-1} \frac{A_{D V}-A_{H 2}}{A_{S}} f_{1}\right.
$$

The quantities of flux that reach the detector are easily summed to

$$
I_{1}=I_{0} \rho_{S}^{3} \frac{A_{S H}}{A_{S}}\left\lceil\frac{A_{D V}-A_{2}}{A_{S}}\right] f_{1}\left[\frac{1}{1-\rho_{S} \frac{A_{S H}}{A_{S}}}\right]
$$

which is the flux that reaches the detector when the detector does not view the first reflection.

Case 2. The detector views the first reflection. In this case, the only additional flux impinging on the detector is that due to the first reflection, which from equation (II), is

$$
R_{1}^{\prime \prime}=I_{0} \rho_{S} f_{2}
$$

where $f_{2}$ is the configuration factor from the area 1lluminated by the beam to the detector sensing element. Since this is the only additional flux impinging on the detector, the total flux reaching the detector for this case 18

$$
I_{2}=I_{1}+I_{0} \rho S P_{2}
$$


The ratio of the flux reaching the detector for these two cases is

$$
\frac{I_{1}}{I_{2}}=\frac{I_{1}}{I_{1}+I_{0} \rho_{S} f_{2}}=\frac{1}{1+\frac{I_{0} \rho_{S} f_{2}}{I_{1}}}
$$

which upon substitution from equation (16) becomes

$$
\frac{I_{1}}{I_{2}}=\frac{1}{I+\frac{\left(f_{2} / f_{I}\right)\left[A_{S}-\rho_{S} A_{S H}\right] A_{S}}{\rho_{S}\left(A_{S H}\right)\left(A_{D V}-A_{H 2}\right)}}
$$

Therefore, the ratio of the detector readings when 1 does and does not Vlew the 1Iluminated area is known for a completely dfffuse sphere coating. Thus, an easily performed test can 1llustrate the quality of a given sphere coating with regard to its reflectance-diffuseness combination, to the uniformity of costing reflectance throughout the sphere, and to whether the cavity used is close enough to a sphere in shape.

Data will be taken with the sulfur-coated sphere shown in F1gure 42 at wavelengths from 1.5 to $10 \mu$. The data should give a quantitative evaluation of the utilfty of sulfur as an integrating sphere coating in the infrared.

\section{3c Calibration of Reflectometer}

The ellipsoldal mirror reflectometer is capable of both absolute and relative measurements of near normal hemispherical reflectance in the infrared. Figure 44 11lustrates the several configurations which are used to get the raw data. Io is the incldent flux, which strikes the specimen at $7^{\circ}$ from the normal. When the detector is placed at the first focal point, the incident flux is measured; when the detector 1s placed at the second focal point and the sample at the first, the flux reflected by the specimen is measured. The ratio of these two readings gives the reflectance of the specimen, except for the flux reflected from the specimen that does not reach the detector. In order to attain a high degree of accuracy, the losses must be accounted for precisely. A convenlent method of accounting for such losses is to prepare an energy balance for the system. The terms for such an energy balance are defined and discussed below.

A) Mirror Losses. Flux is absorbed by the mirror; therefore, the reflectance of the mirror coating must be known. This reflectance will vary with the angle of incidence, and, hence, with position on the mirror, from $0^{\circ}$ at the apex to about $35^{\circ}$ at the edge. There will aiso be some polarization of the reflected flux, which may affect the reading. There wlll be some loss from scattering, due to scratches, dust, and other imperfections on the mirror.

B) Hole Losses. Some of the reflected flux w1ll escape through the hole in the mirror, which admits the incident beam. Th1s loss varleg with the geometric distribution of the reflected flux, and there is no accurate general method for estimating its magnitude.

c) Sample Shielding. Flux leaving the sample normal to its surface w1Il be reflected back to the sample, and, hence, be blocked from reaching the detector. Most of this will be lost, but a very small fraction wlll be reflected by the specimen and reach the detector.

D) Diffuser - Ellipsoid Interreflections. Some of the flux incident on the diffusing sphere w11l be reflected back out the entrance of the sphere to the ell1psold and back to the sphere. ThIs error will be largest when the detector is at the first focal point of the ellipgoid. 
For the reflected beam, much more information is needed. First, a measurement is made with only the sample in the first focal plane, as in Figure 44-2. The measured flux is then a rough estimate of the reflected flux, which must be corrected for mirror losses, hole losses, sample shading, atmospheric absorption, and interreflections between the diffuser and the ellipsoidal mirror. The arrangement shown in Figure 44-3 is used to obtain additional information about the flux incident on the ellipsoidal mirror near the hole. There is a one-to-one correspondence between areas on the ellipse and areas in the first focal plane. Figure 45 shows the projection of the hole, sample, and specularly reflected image in the first focal plane. The hole loss and specimen shielding loss can be computed from the flux incident on these areas. Shield B (Figure 45) is inserted into the first focal plane, and the detector reading is taken as $R_{2}$. The reading without any shield is $R_{1}$. Then $R_{3}=R_{1}-R_{2}$ is a measure of the flux striking shield B. If we assume that the flux is uniformly distributed over the solid angle represented by the shield, or varies linearly over the solid angle, we can correct for hole and sample shielding losses as follows. The total flux striking the shield, of area $A_{H S}^{\prime}$, is $R_{3}$, but the flux that would have struck the area $A_{H}^{\prime}$, the projected area of the hole, has passed out the hole. Thus, the flux density, assuming uniform distribution, is $R_{3} / A_{H S}^{\prime}-A_{H}^{\prime}$, and the corrected $R_{3}$ would be ( $\left.R_{3} A_{H S}^{\prime}\right) /\left(A_{H S}^{\prime}-A_{H}^{\prime}\right)$. The sample also shades the detector with an area $A_{S}^{\prime}$, which, if we assume it to have the same flux density incident on it from the ellipsoidal mirror, will increase the corrected $R_{3}$ to

$$
\mathrm{R}_{3} \frac{A_{H S}^{\prime}+A_{S}^{\prime}}{A_{H S}^{\prime}-A_{H}^{\prime}} \text {. }
$$

The corrected reading, $R_{c}$ will then be

$$
R_{c}=R_{2}+R_{3} \frac{A_{H S}^{\prime}+A_{S}^{\prime}}{A_{H S}^{\prime}-A_{H}^{\prime}} .
$$

Some of the flux that strikes the sample a second time will be reflected to the ellipsoidal mirror and the detector, and thus increase the $R_{I}$ and $R_{2}$ readings. However, this correction is considered at this time to be negligibly small. It will be accurately evaluated in the near future.

The signals read by the detector in the configurations shown in Figure 44-2 and 44-3 will be high, due to interreflection between the diffuser, sphere, and specimen. Computations indicate that the error should be on the order of 2 to 3 percent. Since there does not appear to be any convenient way to eliminate the error, it will be necessary to evaluate the error experimentally and correct for it.

No absolute measurements are being made at this time in the wavelength ranges where atmospheric absorption is significant. Where the error is significant, absolute measurements will be made in a non-absorbing atmosphere. The error cancels out in comparison measurements, hence a standard that has been calibrated in a non-absorbing atmosphere will be used for such measurements.

The remaining major correction is for mirror losses. The coating on the ellipsoidal mirror is aluminum, applied in one second at a pressure of $10^{-5}$ torr to a thickness sufficient to be opaque. There is no overcoat. The reflectivity thus should approach that of pure evaporated aluminum, according to Bennett, et al [10], and Hass [11]. However, there is some scatter, due to imperfections in the finish on the mirror, which will increase the apparent absorption. Hence it will be necessary to measure the effective reflectance as a function of location on the mirror. This will be done as follows: a gold mirror and an aluminum mirror, of known reflectance and minimum scatter, will be used as samples. They will be mounted on a specimen holder that can be tilted to any angle in any direction, so that the specularly reflected beam can be directed to any part of the ellipsoidal mirror. Measurements of the reflectance of the samples will thus give the data required to evaluate the reflectance of the mirror in the illuminated area. Measurements will be made at all wavelengths of interest. If significant variation in reflectance is found over the surface of the mirror, it can be corrected for. Effects of polarization by the ellipsoidal mirror will also be considered.

The losses due to shading by the sample holder and support are believed at this time to be negligibly small. This will be verified experimentally. 
All of the errors discussed above will be present when the instrument is used for absolute measurements - i.e., when the incident flux is measured directly. If comparative measurements are made - i.e., the incident flux is evaluated by measuring the reflectance of a standard of known reflectance, as indicated in Figure 44-2, 3, 4, and 5 - the corrections tend to cancel out unless there is a large difference in the geometric distribution of the flux reflected by the sample and standard.

\section{Summary of Work on Relation of Thermal Emittance to Other Properties}

\subsection{Introduction}

One of the long-range goals of this program from the beginning has been to obtain a better understanding of the fundamental processes involved in the interaction of electromagnetic radiation with matter. Attainment of such a goal is intrinsically desirable; further, it can contribute to the attainment of other more immediate objectives in two ways. First, such a knowledge can aid in the development and evaluation of equipment and techniques for measuring the thermal radiation properties of materials. Second, if meaningful equations can be found or derived that relate thermal radiation properties to other more easily evaluated properties of materials, they can be used to interpolate and extrapolate thermal radiation property data, obtained at a relatively few temperatures and wavelengths, over much wider temperature and wavelength ranges.

Pertinent information on this subject has been published by many authors, representing widely different scientific disciplines. However, there does not appear to be any single compilation of the major contributions. This summary includes a review of the pertinent literature, including a discussion of the limitations of the various theoretical treatments. In addition, the optical behavior of rhodium, for which reliable data are available, is treated in some detail.

\subsection{Consideration of Quantum Theory [12]}

Quantum theory is used to explain on an atomic or sub-atomic scale the generation and absorption of electromagnetic radiation. According to this theory, electromagnetic radiation consists of discrete particles called photons, each of which has a given kinetic energy, which is related to frequency. These photons are generated and absorbed as units, by statistical processes. They travel in straight lines, at a velocity, $c$, which in vacuum is a fundamental natural constant, the speed of light. Their direction of propagation is changed in a predictable manner in passing through an interface separating two volumes having different optical properties.

Because the generation and absorption of photons are statistical processes, it is not possible to predict when or where either process will occur, but it is possible to predict statistically what fraction of the photons passing through a material will be absorbed in unit path length, and how many photons of a given energy will be generated per unit volume and unit time for any given material under given conditions.

Quantum theory has been used successfully to account quantitatively for the emission and absorption of gases, and qualitatively to account for certain features of the spectra of liquids and solids. In general, however, the mathematical treatment is too cumbersome to permit computation of the complete spectra of solids and liquids, even by use of modern computer techniques.

Classical electromagnetic wave theory, on the other hand, can be used to explain the radiant heat transfer on a macroscopic scale. While it does not explain the specific mechanisms involved in the generation and absorption of individual photons, it does account quantitavely for the net exchange of energy by radiation. Hence the discussion that follows will be based primarily on classical electromagnetic wave theory. 
Every object at a temperature above $0^{\circ} \mathrm{K}$ is continually emitting radiant energy. This energy leaves the object in the form of waves, which are characterized by an electric field, a magnetic field, polarization, and frequency. The electric and magnetic fields are at right angles to each other and to the direction of propagation. Polarization refers to the orientation of the electric field; in a plane polarized beam of light the electric fields of all the waves are parallel. The wave travels in free space at the velocity of light, c. The nature of the wave is mathematically described by the Maxwell equations, which can be found in any standard text on light.

Let us consider a plane interface separating free space from a volume consisting of some optically homogeneous material as yet unspecified. As an electromagnetic wave impinges on this interface from free space, interaction of the wave and material will occur, and the character of the wave will change. It will be separated into two components, one reflected by the interface and one continuing into the material. We need not be concerned with the mechanism of the change, but only to quantitatively describe it and relate it to the properties of the material.

The change in the continuing component is most simply described in terms of phase and velocity of propagation. The wave within the material is described by

$$
E=E_{0} \exp (j \omega t-\gamma x)
$$

in which $E$ is the amplitude of the electric field at time $t, E_{0}$ is the amplitude at zero time, the term exp ( ) indicates that e, the base of natural logarithms, is raised to tha indicated power, in parenthesis, $j=\sqrt{-1}$, $w$ is frequency, $\gamma=\alpha \div j \beta$, where $\alpha$ is the attenuation factor and $\beta$ is the phase factor, and $x$ is the distance traversed in time t. If zero time is taken as the time the wave impinges on the interface, $E_{O}$ is the value for the wave in free space.

The reflected energy can be computed by using boundary conditions compatible with continuity of the field vector components. This approach develops the Fresnel equations for the two reflected components that are polarized normal and parallel to the plane of incidence

$$
\begin{aligned}
& \rho(\Psi, \varphi, \lambda)_{\mathrm{n}}=\left[\frac{\cos \Psi-Z \cos \varphi}{\cos \Psi+2 \cos \varphi}\right]^{2} \\
& \rho(\Psi, \varphi, \lambda)_{P}=\left[\frac{\cos \varphi-Z \cos \Psi}{\cos \varphi+2 \cos \Psi}\right]^{2}
\end{aligned}
$$

in which $\rho=$ reflectance, the subscripts $\Psi, \phi$ and $\lambda$ denote angle of incidence, angle of refraction and wavelength, respectively, and the subscripts $n$ and $p$ denote the components polarized normal and parallel to the plane of incidence. $Z$ is the impedance ratio of the material, $Z=Z * / Z_{0} *$, where $Z *$ is the intrinsic impedance of the material and $Z_{0} *$ is that of free space. The angles, as are all angles in optics and wave theory, are measured from the normal to the surface to the direction of propagation. The direction of propagation of the reflected ray is always in the plane of incidence, and the angle of reflection is equal to the angle of incidence, but lies on the side of the normal opposite the incident ray. The angles $\Psi$ and $\varphi$ are related by Snell's law

$$
\sin \varphi=|z| \sin \psi
$$

It is evident that the reflectivities given by equations (23) and (24) are not complex quantities, but are real numbers, and as such the value of the reflectivity at a single wavelength and angle of incidence cannot completely describe the reflection process. To evaluate $Z$ or $Z *$ from measured reflectivities requires information at various angles. Even then, the computation requires a tedious trial and error approach and precise information on the angular dependence of the reflectivity data. 
Further manipulation of wave relations and the Maxwell equations involves complex parameters (with real and imaginary components) that describe the wave after traversing a known path length in the material. A list of parameters that have been used for this purpose is given in Table 5, with a brief explanation of their physical significance.

To obtain emissivity values for opaque materials, Kirchhoff's law is applied to give

$$
c(\varphi, \lambda)=1-\rho(\varphi, \lambda)
$$

where $\epsilon(\varphi, \lambda)$ is the spectral emittance at wavelength $\lambda$ and $\operatorname{direction} \varphi$, and $\rho(\varphi, \lambda)$ is the spectral specular reflectance for radiation incident at angel $\varphi_{\text {. }}$

A special case of great interest (and less complexity) is when $\varphi=0$, giving

$$
c_{n}(0, \lambda)=\epsilon_{p}(0, \lambda)=1-\left[\frac{1-z}{1+z}\right]^{-2}
$$

Equation (27) can also be written in terms of indices of refraction and absorption

$$
c(0, \lambda)=\frac{4 n}{(n+1)^{2}+n^{2} k^{3}}=1-\left[\frac{n *-1}{n *+1}\right]^{3}
$$

where $n$ is index of refraction, $K$ is absorption index, and $n *$ is the complex index of refraction, $n^{*}=n(l-j k)$. Typical values of these parameters are given in Table 6 .

So far, no assumptions have been made as to the nature of the material, except that it is optically homogeneous and isotropic, with a perfectly smooth interface.*

$$
4.4 \text { Metals }[12,13,14]
$$

In the preceding section of treatment of the relation of thermal radiation properties of materials was developed on the basis of complex factors, without consideration of the nature of the material. To evaluate any of the parameters given in Table 5, it is necessary to examine in detail the effect of the impinging electromagnetic wave upon the electron configuration of the material. The following discussion is confined to metallic conductors, and is limited in scope to theories within the framework of classical electron physics.

\section{4 a Free Electron Models}

The earliest attempts to predict the optical properties of metals were made by Iorenz [15], Drude [16], Kronig [17], and Mott and Zener [18], who assumed the metal to contain electrons which were essentially free to move under the influence of the electric field induced by the incident electromagnetic wave. These free electrons are the valence electrons in the outer shell of the atoms constituting the metal. When the wave is incident upon its surface, an oscillating electric field parallel to the surface is induced in the metal. The free electrons will oscillate under the influence of this field at the frequency of the incident wave. There is a phase difference between the oscillation of the electrons and that of the field, caused by a viscous damping force, $\gamma_{f}$, arising from the collisions between accelerated electrons and the atomic lattice.

The equation of motion for an electron moving under these conditions is:

$$
m y^{\prime \prime}+2 \pi m \gamma_{f} y^{\prime}=q_{0} E \exp (j \omega t)
$$

where $\mathrm{m}$ is the mass of an electron, $\mathrm{y}^{\prime}$ is velocity, $\mathrm{y}^{\prime \prime}$ is the acceleration, qo is the charge on an electron, $E$ is the amplitude of the electric field, exp ( ) indicates that the base of natural logarithms is raised to the power in parentheses, $j=\sqrt{-1}, \omega$ is frequency and $t$ is time. The current density corresponding to this electronic motion can then be computed, which gives the relationship between the parameters descriptive of the wave and the parameters descriptive of the electronic configuration. The most popular form is in terms of the

* It is also assumed that the magnetic effects are negligible compared to the electric effects, and that the permeability, $\mu *$, of the material is the same as that of free space. These are reasonable assumptions for most materials of primary concern here. 
dielectric constant, $K^{*}$

$$
K *=1-\left(\lambda / \lambda_{0}\right)^{3} \frac{1+j \frac{\lambda}{\lambda_{f}}}{1+\left(\lambda / \lambda_{f}\right)^{2}}
$$

where $\lambda t$ wavelength of Incident wave, $\lambda_{0}=c / q_{0}\left(\frac{\pi m}{N_{f}}\right)^{\frac{1}{2}}, c=$ speed of 11 ght, $N_{f}=$ mumber density of free electrons per unit volume, $\lambda_{f}=2 \pi c \tau,=c \lambda_{f}, \tau=$ relaxation time.

In order to predict the optical properties, two parameters of the material must be evaluated, $T$ and $\mathrm{N}_{f}$. If $\mathrm{N}_{f}$, the number of free electrons excited by the induced field, is considered to be the number of valence electrons per unit volume, the relaxation time, $T$, can be computed from electrical conductivity and the assumption of a spherical Ferml surface. This approach gives favorable results compared with measured values for liquid metals, but fails for more complex metals. Figure 46 shows the general shape of the computed and measured emissivity and optical constants for mercury, for which the theory gives fair agreement.

If it is assumed that the phase change arising from electronlc collisions can be neglected, the theoretical model is greatly simplified, and the optical constants and the emissivity can be expressed in terms of the d.c. electrical resistivity alone. Such an assumption was made in the Drude model referred to above at very long wavelengths, or for conditions where the reloxation time is infinite. The resulting equation for these conditions is the celebrated Hagen-Rubens [.19] (sometimes called the Drude Zener) relation:

$$
\in(0, \lambda)=0.365\left(p_{m} / \lambda\right)^{\frac{3}{2}}-0.0464\left(p_{m} / \lambda\right)
$$

where $\varepsilon(0, \lambda)$ is the normal spectral amlssivity and $\rho \mathrm{m}$ is the d.c. electrical resistivity. The usefulness of equation (3I) has long been recognlzed, but its limitations have not always been appreclated. Comparison of values computed from thls equation with experimental data reveals differences which vary with the metal involved, the wavelength and the temperature. These differences arise because the assumptions made in deriving the equation are not completely valid, particularly at short wavelengths.

Many other equations have been derived on the basis of equation (31), for computation of hemispherical, total and directional emissivity. Table 7 lists the equations, together with a brief explanation of their limitations.

\section{4b The Multi-Free/Bound Model}

The failure of the free electron model to yield computed values that agree with experimental data in the wavelength regions of interest should not be interpreted as a failure of the classical approach, but rather should be ascribed to the over-simplification of the mechanism of the reflection (or absorption) process. Quantum theory indicates that both free and bound electrons exist in a metal, and both types of electrons exlst in different energy states. As such, they react in different degrees to the influence of the electric fleld induced by the incident radiation. The free electrons of a particular energy state are characterized by a particular viscous damping parameter, $\lambda_{f}$ (or relaxation time $\tau$ ), and number density, $N_{f}$.

Contributions due to the influence of the induced electric field on bound electrons must also be considered. They can be assumed to be elastically bound in their equilibrium position and each type of bound electrons is characterized by particular values of three parameters elastic restoring force, $K_{b}$, viscous damping force $\lambda_{b}$, and number density, $N_{b}$. Then a clsssical model for the most general case, where contributions due to several types of free and bound electrons must be considered, will have the following equations of motion.

$$
\begin{aligned}
& \text { Free electrons, type } g \quad g=1,2,3, \ldots \\
& \quad \operatorname{myg}_{g}^{\prime \prime}+2 \pi m \frac{c}{\lambda_{f_{g}}} y_{g}^{\prime}=-q_{0} \Sigma_{0} \exp (j \omega t)
\end{aligned}
$$




$$
m_{h}^{\prime \prime}+2 \pi m \frac{c}{\lambda_{b h}} y_{h}^{\prime}+k_{b h} y_{h}=-q_{0} E_{0} \exp (j \omega t)
$$

Here, $\mathrm{y}$ is electron displacement. The development of the optical properties from the equations of motion proceeds as described above for the simpler free electron model. The resulting form for the complex dielectric constant, $K^{*}$, is

$$
K *=1+\frac{\lambda^{2} q_{o}^{2}}{4 \pi^{3} \varepsilon_{0} c^{3}}\left[\frac{N_{b h} \lambda_{o h}^{2}}{\lambda^{3}-\lambda_{o h}^{3}+j \frac{\lambda_{o h}^{2}}{\lambda_{b h}}}-\frac{N_{f g} \lambda_{f g}}{\lambda_{f g}-j \lambda}\right]
$$

$$
\begin{aligned}
& \text { Where } \lambda_{f g} \text { = viscous damping parameter for fres electrons, type } 8 \\
& \lambda_{\text {bh }}=\text { viscous damping parameter for bound electrons, type } h \\
& \mathrm{~K}_{\mathrm{bh}}=\text { elastic restoring force on bound electrons, type } \mathrm{h} \\
& \lambda_{\mathrm{oh}}=2 \pi \mathrm{c} \frac{\mathrm{m}^{\frac{1}{2}}}{\mathrm{~K}_{\mathrm{bh}}} \text {, resonant wavelength of bound electrons, type } \mathrm{h} \text {, with no damping forces } \\
& \text { present. } \\
& \varepsilon_{0}=\text { permittivity of free space }
\end{aligned}
$$

In order to evaluate the optical properties by means of these cumbersome equations, 2 method for evaluating $\lambda_{f}, \lambda_{b}, K_{b}$ and $\lambda_{o}$ must be found. So far no theory has been developed for estimating these parameters from the electronic configuration of the metal. Such an approach would require quantum mechanical treatment, or comparison with experimental data.

Roberts $[20,21,22]$, using the experimental data approach, successfully determined parameters for a number of metals. The form of the equation is somewhat different than that of equation (34), but a comparison of the forms and meanings of equivalent parameters is essily seen in Tables 8 and 9. We will not discuss the method used to evaluate the parameters from experimental data, but the results are shown in Table 10.

The success of the Roberts treatment in producing computed emissivity values that agree in detail with experimental data has been substantial. Inflection points and "humps" have been properly described, so that if the material parameters are known, optical behavior is completely described in the wavelength region considered. No equations have yet been developed on the basis of the multi-free/bound electron model that correspond to those developed for the simpler free electron model. It is apparent that until equations are developed that include the contributions from resonance effects, accurate prediction of emissivity from structural factors (or good agreement between computed and experimental data) is not to be expected, particularly at the shorter wavelengths (higher frequencles).

\section{4c The Kramer-Kronig Relation [23]}

It was stated above that two parameters are required to completely describe the optical behavior of a material at a given wavelength. The Kramer-Kronig relation provides an alternate method of obtaining full knowledge of the optical behavior from knowledge of only one parameter (such as the normal spectral reflectivity) at all wavelengths, or, more precisely, over the wavelength range where variations in the property occur. This technique is a very powerful tool in solid state physics studies, and is amply described and demonstrated in the literature $[24,25]$. A brief derivation is given in Appendix A. 
The Kramer-Kronig equation has the form:

$$
\theta\left(\lambda_{0}\right)=\frac{1}{\pi} \int_{0}^{\infty} \frac{v_{0}(\lambda)}{v(\lambda)} \ln \left|\frac{\lambda+\lambda_{0}}{\lambda-\lambda_{0}}\right| d \lambda
$$

where $\theta\left(\lambda_{0}\right)$ is the phase component of the complex quantity, $V$, which has the real part $v\left(\lambda_{0}\right)$ :

$$
V=v \exp (j \theta)=\frac{n *-1}{n *+1}
$$

and from previous relations (eq. 28) it is apparent that $\rho(0, \lambda)=V^{2}$.

To compute the phase $\theta\left(\lambda_{0}\right)$ at $\lambda=\lambda_{0}$, equation (35) is integrated over the entire wavelength region (of consequence) about the pole $\lambda=\lambda_{0}$. Knowing the phase it is then possible to solve for the real and imaginary parts of $n^{*}$.

The greatest difficulty encountered in using this technique is to establish the wavelength range over which the integral must be evaluated. At first thought one might expect that to evaluate the phase at some longer wavelength, such as $10 \mu$, contributions to the integral within the ultraviolet region may be ignored. In general this is not true, as can be verified upon closer inspection of the form of the integral. As a rule, full knowledge of the reflectivity spectrum is required. The lower limit of the significant range is reached when the reflectivity goes monotonically to a zero value as the wavelength approaches zero. The upper limit is reached when the reflectivity becomes constant or approaches unity as the wavelength increases. The important consequence of this is that the integral must be evaluated at all wavelengths (especially in the short-wave region) where optical activity occurs such as absorption edges or resonance effects.

\subsection{Optical Behavior of Rhodium}

Very early in this study, data on the normal spectral reflectivity of rhodium were collected [26]. The choice of rhodium was based upon the availability of reliable data over a wide wavelength range. The reflectiveity spectrum from $0.058 \mu$ to $10 \mu$ was felt to be adequate information, and also showed interesting inflection points in the wavelength region of significance from a thermal radiation standpoint.

Using the multi-free/bound electron model similar to that of Roberts, attempts were made. to determine the values of the parameters which would give a reflectivity spectrum, figure 47 , comparing favorably with the rhodium data. In attempting to fit the observed values with a formula containing just one free-electron term, the coefficients of that free electron term were determined first to produce a good fit at the upper end of the data- near $\lambda=10 \mu-$ and in the neighborhood of $\lambda=0.25 \mu$, where the reflectivity rises sharply. Then boundelectron terms were introduced, one at a time, to allow fitting of the "humps" in the measuredreflectivity curve, such as the curve near $0.45 \mu$ Each time a new term was introduced, the parameters of the older terms had to be modified so as to maintain the previously achieved fit; this in turn necessitated modifying the parameters of the new term, which led to further modifications of the old terms, etc. It was originally thought that the effect of each boundelectron term would be quite local*, so that these modifications would be small, and after one or two modification cycles a good fit would be obtained both at the newly-considered "hump" and at all locations previously considered. Th1s was only partly borne out; a bound-electron term introduced to account for an apparent slight hump between $1 \mu$ and $2 \mu$ had large effects as

* It can be easily shown that the effect of a resonance about a wavelength $\lambda=\lambda_{\mathrm{si}}$ is not necessarily localized, but the influence may extend to other portions of the spectrum. An example of this is shown in Figure 48 where the resulting emissivity is shown including successively more terms. Close inspection reveals how the influence of an additional bound term gives rise to a widespread effect. This particular example is for tungsten at $1800^{\circ} \mathrm{K}$ using the parameters determined by Roberts. 
far down as 0.16u. The comparison of various attempts with the experimental data is shown in Figure 47 and numerical values of the parameters are recorded in Table 11.

It is seen from Figure 47 that curves 1, 2, and 3 compare favorably with the data except at wavelengths near and below 0.74 . The optical constants computed from the parameters in the visible portion of the spectmum (where the computed curve shows good agreement with experiment. al data) do not agree with messured optical constants for the same wavelengths. Hence it is evident that the parameters are mathematically expedient, and have no physical significance.

The Kramer-Kronig relations can be used to obtain the phase and the optical constants of a material at any particular wavelength from the normal reflectivity of the meaterial over the full wavelength spectrum. When the observed values of $V=\sqrt{\rho(0, \lambda)}$ for the wavelength range 0.14 to $10.0 \mu$ were used with equation (36) to compute the phase and optical properties, unrealistic values were obtained. The computed value of $\theta(\lambda)$ at $0.546 \mu$ was 0.274 , while the experimentally determined value is 0.395 . The discrepancy is due to the absence of data on $V$ at wavelengths below $0.14 \mu$. Rough calculations using assumed shapes for the reflectivity curve below $0.14 \mu$ showed that the contribution of this portion of the spectrum to the value of $\theta\left(\lambda^{\prime}\right)$ is apt to be of the same order of magnitude as the contributions of the entire spectrum at wavelengths beyond $0.14 \mu$.

This observation further implies that the attempt to fit the reflectivity curve by use of the general classical model could not fuliy succeed because of the lack of reflectivity data at very short wavelengths. It is apparent that there are "humps" in the reflectivity curve at wavelengths below $0.14 \mu$; the bound-electron terms that should be introduced into the equations to account for these humps would modify the parameters of the free-electron terms previously considered. However, since the locations and shapes of these humps are not predict. able from the reflectance data at longer wavelengths, it is impossible to take them into account so that the parameters would be physically significant.

\subsection{Summary and Conclusions}

This brief review of the fundamentals of wave-matter interaction and the optical behavior of metals has focused attention upon three important aspects of theoretical predictions of thermal radiation properties.

(1) A complete description of the optical behavior requires measurement or prediction of two parameters at any wavelength since two parameters completely characterize the form of the electromagnetic wave interacting with the material.

(2) The thermal radiation property reflectivity (or emissivity) is a thermodynamic property resulting from energy conservation considerations and one such value at a wavelength is not sufficient information to completely describe optical behavior.

(3) The Kramer-Kronig relation provides a means of determining optical behavior from reflectivity, provided data is avaliable over the portion of the spectrum where activity is significant.

The classical models discussed have linttations whlch arise from over-simplification and improper application rather than lack of physical credence. The multi-free/bound electron model as shown by Roberts has been successful in every instance where a comparison with data has been made. This model suffers from its cumbersome form and large number of parameters, giving an appearance of mathematical expediency rather than physical reality. Additional tests of this model require optical constants data or complete reflectivity spectra. The early attempts in this program to determine the model parameters from an incomplete reflectivity spectrum can now be judged as unfruitful and the parameters thus obtained are matherstically expedient and without physical significance.

The spectral region of importance to thermal radiation applications rarely extends to wavelengths as low as $0.2 \mu$, since only 0.18 percent of the energy from a blackbody radiator at $3500^{\circ} \mathrm{K}$ is at shorter wavelengths. In fact, much of the data on thermal radiation properties of materials do not extend below the lower limit of visible light, $0.4 \mu$. 
There is a paradox which should be of concern to workers in the field. For practical applications, data are required from the visible out into the far infrared, while for theoretIcal computations, data are required over a wavelength range extending into the extreme vacuum ultraviolet. Measurements in the vacuum ultraviolet wavelength range, require spectal

experimental techniques. For instance, surface contamination may exert a controling influence on the results obtained. It is not possible to obtain reliable data simply by extending present techniques used at the longer wavelengths.

The alternative approach to specifying optical behavior is to measure two parameters at each wavelength of interest, as was previously suggested. This would require different or more elaborate techniques than are now in use, but the benefits would be significant and lastlng.

\section{Working Standards of Normal Spectral Emittance}

\subsection{Background}

Three sets of working standards of normal spectral emittance have been prepared and calibrated. Set 1 consisted of specimens of platinum- $13 \%$ rhodium alloy and oxidized Inconel. Data on these specimens are given in WADC TR 59-510, part III, [53]. Set 2 consisted of a large number of specimens of various sizes and shapes of commericaliy pure platinum, oxidized Kanthal, and oxidized Inconel. These specimens were originally prepared for distribution by ASD to Air Force contractors, but because of legal questions, they were returned to NBS for sale to the general public. Data on these specimens are given in WADC TR 59-510, part IV [54]. Set 3, like Set 1 , consisted of specimens of platinum- $13 \%$ rhodium alloy and oxidized Inconel. They were prepared for use by RTD. Data on these specimens are given in NBS Technical Note $252[55]$.

\subsection{Comparison of Measured Normal Spectral \\ Emittance of Platinum to Values Predicted by the Hagen-Rubens Equation}

The data referred to above for platinum and platinum- 13\% rhodium alloy working standards were compared with values computed from the resistivity by use of the Hagen-Rubens [19] equation (Drude-Zener). This equation relates normal spectral emissivity, e $(0, \lambda)$, to dc resistivity, $\rho \mathrm{m}$, and wavelength

$$
\epsilon(0, \lambda)=0.365\left(p_{\mathrm{m}} / \lambda\right)^{\frac{1}{2}}-0.0464\left(p_{\mathrm{m}} / \lambda\right)
$$

Equation (31) is applicable at long wavelengths and/or high temperatures. It is derived from classical electromagnetic theory, as discussed in Section 4.49 of this report.

Figures 49 through 51 show the type of agreement observed for platinum. The solid Iines represent the average measured emittances of the six platinum working standards. The vertical Hines indicate the statistical uncertainty, expressed as the total standard deviation, due to arror of measurement and difference in specimens. The two broken lines represent the values computed from equation (31), using resistivity data given by Caldwell [36].

There are some obvious areas of mismatch between the measured and computed values. The maximum near 9 microns at all three temperatures could be caused by (a) bound electron phenom-

(b) an instrumentation error, or (c) an impurity effect. Recent data have shown that is due to a small amourit of silicon impurity in the platinum, as is discussed later.

The rise in emittance values at wavelengths near 15 microns at the higher temperature is pelieved to be an instrumentation error. It has not been found in repeat measurements made on the same specimens after completing certain modification in the spectrometer. Thls is aiscussed in Section 6.4 .

The maxims between 1 and 2 microns have previously been attributed to resonance of bound plectrons, although data reported by Roberts $[20$ indicate that no resonance effect is to be axpected in this wavelength region. The maxima may be due to instrumentation error, since they occur in wavelength reglons where the energy is low. 
At $1100 \circ \mathrm{K}$ (Figure 50) a reasonable fit of the experimental data at wavelengths beyond about 3.5 microns was obtained with the values computed from the resistivity of platinum at 1100 oK given by Caldwell [36]. At 1400 oK, however (Figure 51), a resistivity higher than that given by Caldwell was required to produce even an approximate fit, and at $800 \circ \mathrm{K}$, a resistivity lower than that given by Caldwell was required. These results indicate that oquation (31) cannot be used with published resistivities to extrapolate the measured emittance data to temperatures above $1400^{\circ} \mathrm{K}$ or below $800^{\circ} \mathrm{K}$.

The properties of the platinum- 13\% rhodium alloy are simflar to those of pure platinum. Norma 17y, the presence of an alloying constituent might be expected to introduce changes in the properties. However, these effects are minor and appear as a slight increase in emittance and also in resistivity, as compared to pure platinum. The experimental data are compared in Figures 52 through 54 with values computed from equation (31). The agreement between computed and observed values is about as good as was the case for platinum. In general, however, application of the simplified theory to alloys should be done with caution, since an alloy, in most cases, can no longer be considered as a simple metal.

The observed data show the maximum between 9 and 10 microns that was observed with platinum at each temperature. The maximum between 1 and 2 microns appears only in the platinum$13 \%$ rhodium data at $800 \mathrm{~K}$. The rise in emittance at wavelengths near 15 microns was not observed with the alloy specimens.

Several attempts were made by spectrographic analysis, electron diffraction, and $X-r a y$ diffraction to identify any impurity that might be present in the platinum and platinum- $13 \%$ |rhodium working standards of normal spectral emittance that might account for the peak in the spectral emittance curve near 9 microns. Sillca was suspected, because there is a strong resonance of the S1-O bond at about that frequency. However, these analyses did not show the presence of elements other than platinum or rhodium in the samples.

Finally, an electron probe microanalysis was made [37] of the surface of a platinum-13\% rhodium working standard, which indicated the presence of silicon as a minor constituent, and 1a trace of iron. All other elements of atomic number above 12 were found to be absent.

Area X-ray scanning revealed that the sillcon is strongly concentrated at the grain boundaries, as shown in FIgures 55, 56, and 57, and 1s absent at the rest of the surface. Strong bluish cathodoluminescence observed when the electron beam strikes the surface strongly suggests that the impurity is a non-metallic phase. This observation, combined with the wavelength of the emittance peak, indicates that the impurity is probably present as $\mathrm{S}_{2} \mathrm{O}_{2}$ The amount of the impurity present. cannot be estimated accurately from the measurements, but It is believed to be certainly less than 0.1 percent, and probably less than 0.01 percent.

\subsection{Comparison of Standards Data with Iiterature Values}

Since the optical properties of a metal are markedly affected by the conditions of the surface, differences in values reported by different investigators are to be expected. Recently, the problem of surface characterization of metals [38] has received increasing attention. The complexity of the problem makes it extremely difficult to characterize completely a metal specimen so that data obtained in one laboratory can be reproduced on other specimens of the same material prepared and measured in a second laboratory. In spite of these difficulties, comparison of data obtained on the working standards of normal spectral emittance with data obtained in other laboratories on simflar specimens may serve a useful purpose.

Figure 58 shows values for normal spectral emittance of platinum at $1400^{\circ} \mathrm{K}$ obtained in three other laboratories, together with the data reported for the working standards. The agreement is reasonably good, except that the increase in emittance at 14 to 15 microns was not reported by other investigators and did not occur in more recent measurements discussed in Section 6.4 .

Figures 59, 60, and 61 show values for oxfdized Inconel obtained in other laboratories. together with the data on the working standards. In general, when oxidation times were oquivalent, agreement is satisfactory. 


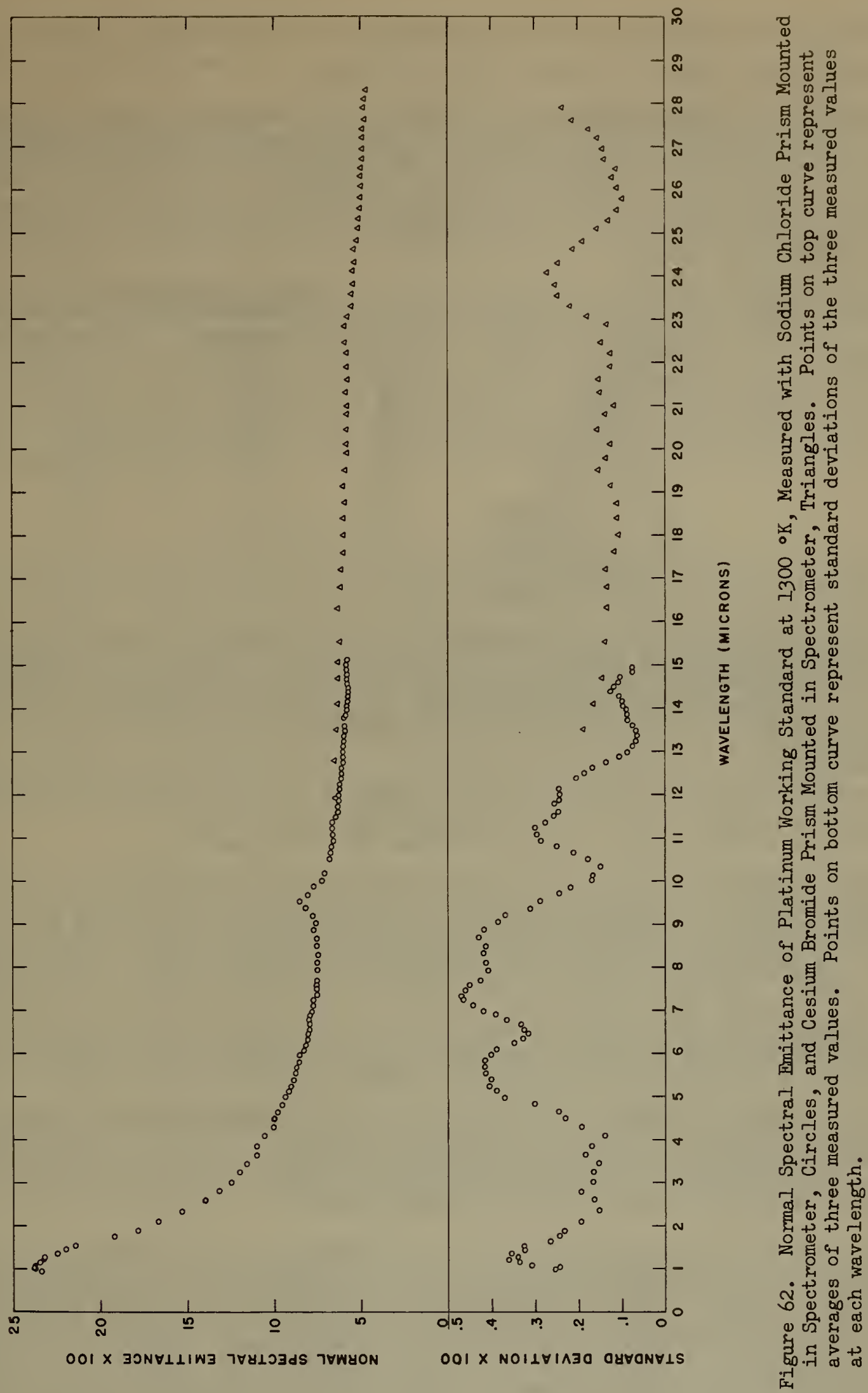


A brief description of the measurements made in other laboratories is given in Table 12 .

\subsection{New Working Standards of Normal Spectral Emittance}

The supply of working standards of normal spectral emittance for sale to the general public became seriously depleted by mid-1964. New specimens of platinum-13\% rhodium alloy, Kanthal, and Inconel were therefore procured and prepared for calibration. The cost of procurement, preparation, and claibration of the new standards is being financed by NBS funds, but because of the great interest of the Air Force in this work, and its close connection to other work on this project, it is being reported here. The specimens were procured in the following shapes and sizes:

No. of Specimens in:

Size
$1 / 2^{\prime \prime}$ discs
$7 / 8^{\prime \prime}$ discs
$1^{\prime \prime}$ discs
$11 / 8^{\prime \prime}$ discs
$11 / 4^{\prime \prime}$ discs
$2^{\prime \prime} \times 2^{\prime \prime}$ squares
$1 / 4^{\prime \prime} \times 8^{\prime \prime}$ strips
$3 / 4^{\prime \prime} \times 10^{\prime \prime}$ strips
$1^{\prime \prime} \times 10^{\prime \prime}$ strips
$1^{\prime \prime} \times 1^{\prime \prime}$ cylinders

Inconel

20

22

20

16

16

15

7

$-$

\section{Kanthal}

20
22
20
16
16
15
7
3
3
-

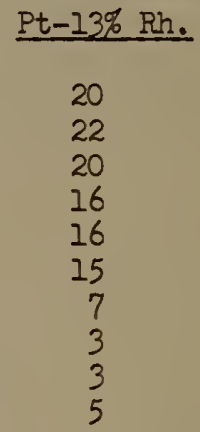

The platinum-13\% rhodium specimens were 0.035 inch thick, the Kanthal specimens 0.043 inch thick and the Inconel specimens 0.053 inch thick. The platinum-13\% rhodium cylinders are to be calibrated at $1400^{\circ}, 1600^{\circ}$, and $1800^{\circ} \mathrm{K}$, by the rotating cylinder procedure. The $1 / 4$ - by 8-inch strips of platinum-13\% rhodium alloy are to be calibrated at $800^{\circ}$, $11000^{\circ}$, 8 nd $1400^{\circ} \mathrm{K}$. The 1/4- by 8-inch strips of Kanthal and Inconel are to be calibrated at $800^{\circ}, 1100^{\circ}$, and $1300^{\circ} \mathrm{K}$.

The platinum-13\% rhodium specimens were washed in hot tap water, then in distilled water, and finally in ethyl alcohol. They were then dried in air and placed in closed containers, supported by the ends or edges only, for storage prior to annealing.

The platinum-13\% rhodium specimens were annealed in a gas-fired furnace. The strip specimens were hung by means of platinum-20\% rhodium hooks from alumina rods in the fumsce. The disc specimens were supported by the edges only on ceramic forms resting on a rigid cerami slab. The square specimens were stood on edge on a flat ceramic form, leaning against a ceramic support. All of the specimens were finally enclosed in a ceramic muffle. The temperature of the furnace was raised to $2830^{\circ} \mathrm{F}\left(1828^{\circ} \mathrm{K}\right)$ over a period of six hours and held at tha temperature for one hour, after which the fuel was cut off and the dampers were closed. The specimens were allowed to cool in the furnace for three days. They were then removed from the furnace and stored in closed containers, supported by ends or edges only.

The Inconel and Kanthal specimens were roughened by sandblasting with 60-mesh fused alumina grit at about $70 \mathrm{psi}$ air pressure, care being taken to obtain a uniformly rough surface on all specimens. They were then cleaned ultrasonicaliy in acetone, rinsed with fresh acetone, and passivated for one minute in 10 percent nitric acid at $109{ }^{\circ} \mathrm{F}$ ( 316 o $\mathrm{K}$ ), rinsed in distilled water and then in freshly distilled acetone.

The Inconel strip specimens were suspended from a metal rack by means of plat1num-20\% rhodium hooks, and the rack was inserted into a cold furnsce. The disc specimens were suppose by the edges only on special ceramic supports placed on the hearth of the fumsce. The square specimens were stood on edge on the hearth of the fumace, leaning against a cerantc support. The furnace, heated by silicon carbide resistance elements, was brought to a tamperature of $19522^{\circ} \mathrm{F}\left(1340^{\circ} \mathrm{K}\right)$ in a period of about 5 hours and held at that temperature for 24 hours, then lowered to $1520 \circ \mathrm{F}(1100 \circ \mathrm{K})$ and held for an additionsl 24 hours, after which the power was cut off and the specimens were allowed to cool in the furnace for two days. They were then removed from the furnace and stored in closed containers, supported by the ends or edges 
oniy. The Kanthal specimens were oxidized following the procedure outlined above for Inconel, except that the heat treatment applied for was 400 hours at $1952^{\circ} \mathrm{F}$ ( $1340^{\circ} \mathrm{K}$ ). The specimens were stored in a similar manner.

Specimens of all alloys were subjected to a minimum of handling after the lnitial cleaning. When handling was unavoldable, metal tongs were used, or rubber surgical gloves were worn, and the specimens were touched only on the ends or edges.

\section{Normal Spectral Emittance}

\subsection{Background}

Equipment and procedures were developed for the measurement of normal spectral emittance of specimens of metal and other materials that could be heated by passing a current through them, at temperatures in the range of $800^{\circ}$ to 1400 , $\mathrm{K}$, and at wavelengths in the range of 1 to 15 microns, as described in WADC TR 59-510, part IV, $\Gamma 54]$. During the preceding contract year, an effort was made to extend the wavelength range out to $35 \mathrm{microns}$ or beyond. Considerable progress was made in modifying the equipment, but satisfactory operation was not achieved. The modifications included (1) constmuction of an air purification system to eliminate or reduce atmospheric absorption, and (2) use of selective filters to reduce errors due to scattered radiation.

\subsection{Modification of Equipment}

A new Reeder vacuum thermocouple detector was procured and installed. The sensitivity of the new detector was about 2.4 times that of the previous detector. The entire optical path from the entrance to the chopper cover to the detector was enclosed in a light-tight and air-tight enclosure. Provision was made to insert or remove filters from the optical path without opening the path to the room atmosphere. It was found there was significant leakage of room air into the recirculating atmosphere control system through the open crankcase of the recirculating compressor. The molsture and carbon dioxide entering the system with the room air soon depleted the drying towers in the air purification system. The situation was corrected by adding a laboratory air drier between the compressor and the air purification train. With this drier in operation, the useful life of the absorbent in the drylng towers of the air purification system has been increased from about 8 hours to over two weeks.

\subsection{Test Procedire}

Preliminary measurements showed that the techniques developed for use in the $1-15$ micron range were not adequate for the $15-40$ micron range. The difficulaties in obtaining reliable data in this long-wavelength range are associated with (a) low energy, (b) scattered radiation within the monochromator, and (c) high atmospheric absorption. All of these difficulties need to be corrected or compensated for, at least in part, if reliable data are to be obtained in this long-wavelength range.

At $1400 \circ \mathrm{K}$, only about $I$ percent of the total energy emitted by a blackbody radiator is at wavelengths longer than 16 microns. At lower temperatures the amount of energy in this wavelength range is less, although the percentage of the total energy is greater. The reduction in energy available for measurement has been compensated for in part by use of the more sensitive Reeder vacuum thermocouple detector. The lower available signal strength resulting from the smaller amount of energy in the long-wavelength range requires that the slit. servomechanism be adjusted to produce a constant $I_{0}$ signal of 5 to $2.5 \mathrm{mv}$, instead of the $10 \mathrm{mv}$ normally used in the $1-15 \mathrm{micron}$ range. This smaller $I_{0}$ signal results in some reduction in precision of the measurements.

The scattered flux within the monochromator is largely undispersed flux from the beam entering the monochromator, and the major portion is at wavelengths near the peak of the Planck distribution function for the temperature of the source. It can be eliminated by placing a selective filter over the entrance aperture to the monochromator. Such a filter should absorb all of the short-wavelength radiation (below 15 microns) and pass all of the long-wavelength radiation." An alternative approach is to replace the diagonal plane mirror near the exit slit of the monochromator with a scatter plate that reflects the long-wavelength radiation specularly, thus focusing it on the detector, while reflecting the short-wavelength flux diffusely, so that most of it is lost before reaching the detector. 
The atmospheric absorption was largely eliminated by removing from the optical path most of the carbon dioxide and water vapor.

Two techniques were developed in an attempt to obtain useful data in the $15-40$ micron wavelength range. In the first technique (A), a black polyethylene filter was placed immediately in front of the entrance slit to the monochromator. This filter absorbs essentially all of the radiation at wavelengths shorter than about $12 \mathrm{microns}$, and transmits 25 to 50 percent of the radiant flux in the $15-40$ micron range. In addition, the slit servomechanism was adjusted to produce an $I_{0}$ signal of $2.5 \mathrm{mv}$ in the constant $I_{0}$ mode, at which setting a constant Io signal was produced while scanning from 15 to about 38.5 microns.

The second technique (B) was to scan the $15-38.5$ micron wavelength range in three portions: approximately 13-27, 27-32, and 32-38.5 microns, respectively. In the first portion, the black polyethylene filter and a nomal $I_{0}$ setting of $10 \mathrm{mr}$ were used. In the second portion, the filter was removed, a $\mathrm{CaF}_{2}$ reststrahlen plate was inserted in place of the plane diagonal mirror, and the Io signal was adjusted to $5 \mathrm{mv}$. The $\mathrm{CaF}_{2}$ reststrahlen plate is a good specular reflector for radiation at wavelengths longer than about 25 microns, but scatters radiation of shorter wavelengths. In the third and final portion, the reststrahlen plate was used, and the $I_{0}$ signal was adjusted to $2.5 \mathrm{mv}$. It was thought that the second technique, while somewhat less convenient than the first, should give greater potentiometer sensitivity in the first two portions, and, hence, probably better precision, but it did not approach expectations.

The test procedures were evaluated by using the combination of a 50 percent transmittance sector-disc attenuator and a laboratory blackbody furnace to simulate a specimen having a normal spectral emittance of 0.50 at all wavelengths. Four tests were made at each of the three temperatures, 800,1100 , and 1300 o , using each of the two procedures. The data were reduced at 23 wavelengths in the 12.4 to 26.8 micron range, 15 wavelengths in the 26.4 to $32.6 \mathrm{ml}$ cron range, and 15 wavelengths in the 32 to 37.65 milcron range.

The data in Table 13 indicate that in the two shorter wavelength intervals, where the $I_{0}$ signal is different for the two procedures, the over-all scatter, measured as the average standard deviation at the 23 and 15 wavelengths, respectively, was smaller for procedure A than for procedure B in three of the six cases, and the data in table 14 show that the accuracy was better for procedure A than for procedure B in all six cases. On the basis of these results, procedure A was selected for use in future measurements in the 15-38 micron range.

\subsection{Measurements on Platinum}

The normal spectral emittance of a platlnum working standard was measured over the wavelength ranges of $1-15$ microns and $13-28.5$ microns, at $1300 \%$ K. Three separate muns were made and the data were reduced as before. The average emittance values and standard deviations are plotted in Figure 62. The curve for the average emittance values shows that the results with the sodium chloride prism $(1-15 \mu)$ and those with the cesium bromfde prism $(12.8-28 \mathrm{H})$ check well within the known precision of measurement in the range where the two curves overlap. The data on the standard deviations of the measured values indicate that the precision of measurement is about the same in the two ranges. The rise in emittance in the 13-15 micron region that was found in the original calibration (Figure 58) was not confirmed in the measurements with either prism (Figure 62).

\section{Sumnary and Plans For Future Work}

Constmuction of a laser-source integrating sphere reflectometer was completed. The sphere coated with $3 \mathrm{M}$ Velvet white paint was calibrated for integrating and reflecting properties at 0.632 microns, and preliminary tests indicated that the shallow cavity technique offers advantages for the calibration of integrating sphere reflectometers. A heater for use at temperatures up to $800 \mathrm{~K}$ was constmucted, and preliminary tests showed that emitted flux from the hot specimen will not invalidate reflectance measurements on specimens at temperatures up to at least $2500^{\circ} \mathrm{K}$, and perhaps $3000^{\circ} \mathrm{K}$. Future plans include (I) modification of the detect. or viewing configur un to reduce errors found with the present configuration, (2) recoating of the sphere with parium sulphate, to replace the $3 \mathrm{M}$ Velvet white paint, (3) calibration of the response of the modified reflectometer at $0.632,1.15$ and 3.39 microns, (4) construction of a specimen heater to operate at temperatures up to $1800 \mathrm{oK}$, and $(5)$ design of a specimen heater to operate at temperaturs up to $3000^{\circ} \mathrm{K}$. 
Calibration of the reflecting properties of the ellipsoldal mirror reflectometer is essentially complete. A preliminary error analysis indicates that the instmument is capable of measuring absolute reflectance at room temperature under conditions approximating normal illumination and hemispherlcal viewing with an accuracy of at least 2 percent, and perhaps 1 percent under ideal conditions. Future work with this instrument will be carried on under a project financed by the National Aeronautics and Space Administration.

A brief review of the literature on the relation of emittance and reflectance to other properties of metals and dielectrics is presented, together with a brief summery of work done in predicting the properties of platinum and rhodium. It was shown that the complete discription of the optical behavior of any material requires evaluation of two parameters at any one wavelength, since two parameters completely characterize the form of the electromagnetic wave interacting with the material. As an alternative, the Kramer-Kronig relation provides a means of determining optical behavior from a knowledge of one parameter, reflectivity, at all wavelengths of the spectrum where optical activity is significant. No further work is planned on the relation of emittance to other properties of materials.

New working standards of normal spectral emittance have been procured on NBS funds and prepared for celibration. These consist of platinum-13\% rhodium alloy, oxidized Kanthal and oxidized Inconel. The work of calibrating these specimens will be completed in the early part of the next contract year.

The normal spectral emittance equipment was modified to operate in the 15 to 37.5 micron region. This completes the development work with this equipment.

\section{References}

[1] Jacques, J. A., and Kuppenheim, H. F. Theory of the Integrating Sphere. J. Opt. Soc. Am. $45,460(1955)$.

[2] Kelly, F. J., and Moore, D. G., A Test of Analytical Expressions for the Thermal Emissivity of Shallow Cylindrical Cavities. Applied Optics, 4. 31 (1965).

[3] Gouffé, A., Rev. Opt. 24, I (1945).

[4] Edwards, D. K., Gier, J. T., Nelson, K. E., and Rodiick, R. D. Integrating Sphere for Imperfectly Diffuse Samples, J. Opt. Soc. Am. 51, 1279 (1961).

[5] Bennett, H. E., and Koehler, W. F., Precision Measurement of Absolute Specular Reflectance with Minimized Systematic Errors, J. Opt. Soc. Am. 50, 1 (1960).

[6] Rohzhin, V. V. A Photomultiplier for Measuring Radiant Flux in Iighted Models with an Absorbing Medium. Izvestis Vysshikh Vchebnykh Zavedeniy, Priborostroyenie, I, 94 (1961).

[7] Birkeback, R. C. Monochromatic Directional Distribution of Reflected Thermal Radiation from Roughened Surfaces. Ph.D. Thesis, Univeristy of Minnesota (1962).

[8] Kronstein, M., Kraushaar, R. J., and Deacle, R. E. Sulfur as a Standard of Reflectance in the Infrared. J. Opt. Soc. of Am. 53, 458 (1963).

Agnew, J. T., and McQuistan, R. B. Experiments Concerning Infrared Diffuse Reflectance Standards in the Range 0.8 to 20.0 Microns. J. Opt. Soc. of Am. 43, 999 (1953).

[10] Bennett, H. E., Silver, M., and Ashley, E. J. Infrared Reflectance of Aluminum Evaporated in Ultra-high Vacuum. J. Opt. Soc. of Am. 231089 (1963).

[11] Hass, G. Engineering Research and Development Laboratories, Ft. Belvoir, Va., personal communication.

[12] Von Hippel, A. R. Dielectrics and Waves, J. Wiley and Sons, New York (1954).

[13] Seitz, F. The Modern Theory of Solids, McGraw-Hill Book Co., New York (1940). 
[14] Harrison, T. R. Radiation Pyrometry and Its Underlying Principles of Radiant Heat Transfer, J. Wiley and Sons, New York (1960).

[15] Lorentz, H. A. Wiedem Ann. 2, 64 I (i880).

[16] Drude, P. Annalon der Physik 64159 (1898), I 566 (1900), 39530 (1890).

[17] Kronig, R. D. L. Proc. Royal Soc. 124409 (1929), 133255 (1931).

[18] Mott, N. F. and Zener, C. Proc. Camb. Phil. Soc. 30, 249 (1934).

[19] Hagen, E., and Rubens, H. Emissionsvermőgen und elektrische Leitfăhigkeit der Metallegiemungen, Verh. Deutsch. Phys. Ges. 6128 (1904).

[20] Roberts, S., Interpretation of the Optical Properties of Metal Surfaces, Phys. Rev. 100 $1667(1955)$.

[21] Roberts, S., Optical Properties of Nickel and Tungsten, and their Interrelation According to Drude's Formula, Phys. Rev. 114 104 (1959).

[22] Roberts, S., Optical Properties of Copper, Phys. Rev. 1181509 (1960).

[23] Stern, F. and Guertin, R. F., Elementary Theory of the Optical Properties of Solids, NOL Tech. Rep. 62-66, Naval Ordnance Laboratory, White Oak, Md. July 1962 AD 298734.

[24] Hunter, W. R., Optical Constants of Metals in the Extreme Ultra-violet. II Optical Constants of Aluminum, Magnesium, and Indium at Wavelengths Shorter than their Critical Wavelengths, J. Opt. Soc. Am. 242081 (1964).

[25] Taft, E. A., and Phillips, H. R., Optical Constants of Silver, Phys. Rev. 1211100 (1961).

[26] Haas, G., Engineering Research and Development Laboratories, Ft. Belvoir, Va., persona] communication.

[27] De Witt, D. P., Ph.D. Thesis, School of Mechanical Engineering, Purdue University (1963).

[28] Aschkinass, E. Ann. der Physik 17, 960 (1905).

[29] Foote, P. D., The Fmissivity of Metals and Oxides, BuI1. NBS 11, 607, (1914-15).

[30] Davisson, D., and Weeks, J. R. The Relation Between the Total Thermal Emissive Power of a Metal and its Electrical Resistivity. J. Opt. Soc. Am. 858 (1924).

[31] Schmidt, E., and Eckert, E. Über die Richtungsverteilung der Wärmestrahlung von Oberflächen. Forschung auf d. Geb. d. Ing. - Wes., Vol. 6, July - Aug. 1935, pp. 175-183.

[32] Parker, W. J., and Abbott, G. C. Total Emittance of Metals. Proc., Symposium on Thermal Radiation of Solids, NASA Special Publication SP 55 (1965).

[33] Schornhorst, J. R. The Emissivity and Emittance of Metallic Conductors, M. S. Thesis, School of Mechanical Engineering, Purdue Univeristy (1962).

[34] Hass, G. Optik 1, 2, (1946).

[35] Wartenberg, H. V., Verh. d. Deutsch. Physik. Ges. 12 105 (1910).

[36] Caldwell, F. R., Thermocouple Materials, NBS Monograph 40, (1962).

[37] Heinrich, K. F. J., Spectrochemical Analysis Section, Analytical Chamistry Division, National Bureau of Standards, personal communication.

[38] De Witt, D. P. Surface Characterization of Real Metals. Proc. of Symposium on Thermsl Radiation Properties of Solids. NASA SP-55 (1965). 
Price, D. J. The Emissivity of Hot Metals in the Infrared, Proc. Phy. Soc. (Iondon) 59. 118 (1947).

Maki, A. G., Stair, R., and Johnston, R. G., Apparatus for the Measurement of the Normal Spectral Fimissivity in the Infrared. J. Res. NBS 64c 99 (1960).

Schatz, E., and McCandless, I. C., Research for High Emittance Coatings, Tech. Doc. Rep. No. ASD TR 62-443, Directorate of Materials and Processes, ASD, A. F. Systems Command, WPAFB, Ohio (1962).

[42] Blau, H. H., Jr., Marsh, J. B., Martin, W. S., Jasperse, J. R., and Chaffee, E. Infrared Spectral Emittance Properties of Solid Materials, AFCLR-TR-60-416, Geophysics Research Directorate, A. F. Research Division, Cambridge, Mass. (1960).

[43] Seban, R. A. Thermal Radiation Properties of Materials, WADD Tech. Rep. 60-370, pt. II $(1962)$.

[44] Slemp, W. S. and Wade, W. R. A Method for Measuring the Spectral Normal Emittance in Alr of a Variety of Materials Having Stable Emittance Characteristics. Proc. Symposium on Measurement of Thermal Radiation Properties of Solids, J. C. Richmond, Ed. p 4h3, NASA SP-31 (1963).

[45] Meier, W. Ann. der Physik, 31 1017 (1910).

[46] Erochin, P., Ann. der Physik, 39213 (1912).

[47] Duncan, R. W., and Duncan, R. C., Phys. Rev. 1294 (1913).

[48] O'Brịen, B., Phys. Rev. 2793 (1926).

[49] Tronstad, L., and Feacham, C., Proc. Royal Soc. (Iondon) A145 115 (1934)

[50] Schultz, I. G. Experimental Study of the Optical Properties of Liquid Hg and Ifquid Ga in the Wavelength Range of $0.23 \mu$ to $13 \mu$. J. Opt. Soc. Am. 4764 (1957).

[51] Harrison, W. N., Richmond, J. C., PIyler, E. K., Stair, R., and Skramstad, H. K. Standardization of Thermal Emittance Measurements. WADC Technical Report 59-510 (March 1960).

[52] Harrison, W. N., Richmond, J. C., Plyler, E. K., Stair, R., and Skronestad, H. K. Standardization of Thermal Emittance Measurements, WADC Technical Report 59-519 Part II. (Feb. 196I).

[53] Harrison, W. N., Richmond, J. O., and Skronestad, H. K., Standardization of Thermal Emittance Measurements, Part III, Normal Spectral Emittance $800^{\circ}$ - 14,00 $\mathrm{K}$. WADC Technical Documentary Report TR-59-510 Part III. (March 1962).

[54] Harrison, W.N., Richmond, J. C., Shorten, F. J., and Joseph, H. S. Standardization of Thermal Emittance Measurements, Part IV. Normal Spectral Emittance, 800 - 14,00 ${ }^{\circ} \mathrm{K}$. Technical Report No. WADC-TR-59-510 Part IV. (Nov. 1963).

[55] Richmond, J. C., De Witt, D. P., and Hayes, W. D., Jr., Procedures for Precise Determination of Thermal Radiation Properties, November 1962 to October 1963. NBS Technical Note 252 (Nov. 1964).

[56] Richmond, J. C., Physical Standards of Emittance and Reflectance, in Radiative Transfer from Solid Materials, Blau, H. H., Jr. and Fischer, H., editors, p. 142-153, Me.cMillan Co., New York (1962). 


\section{Appendix $A$}

Kramers-Kronig relation for phase in terms of reflectivity.

If $V=\frac{n-n j k-1}{n-n j k+1}$ is, as a function of the wavelength $\lambda$, analytic throughout the lower half-plane, and is never zero there, then we can define $f(\lambda)=\operatorname{lnV}$ to be analytic in the lower half-plane. If $\lambda_{0}$ is any positive real number, let $C_{A}$ be the contour illustrated, where the large semicircle has radius $A$ and the mall one radius $1 / A$.

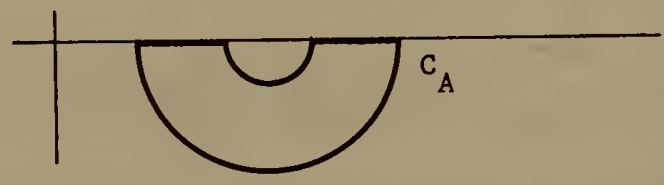

Call these semicircles $C_{A}^{\prime}$ and $C_{A}^{\prime \prime}$ respectively, and call the straight part of the contour $C^{\prime \prime}$. By the Cauchy integral theorem

$$
\int_{C_{A}} \frac{f(\lambda)}{\lambda-\lambda_{0}}=0=\int_{c_{A}^{\prime}}+\int_{c_{A}^{\prime \prime}}+\int_{c_{A}^{\prime \prime \prime}}
$$

If $f(\lambda)$ approaches zero uniformly as $\lambda$ approaches infinity, it is clear that the first integral goes to zero as A increases. The second Integral is, for large A, approximately

$f\left(\lambda_{0}\right) C_{c}^{\prime \prime} \frac{d \lambda}{\lambda-\lambda}$, and so reduces to $j \pi f\left(\lambda_{0}\right)$. The third integral approaches the negative of the Cauch principal value of the integral of $\frac{f(\lambda)}{\lambda-\lambda_{0}}$ from $-\infty$ to $\infty$, which we denote $P \int_{-\infty}^{\infty} \frac{f(\lambda)}{\lambda-\lambda_{0}} d \lambda$.
We thus have

$$
f\left(\lambda_{0}\right)=+\frac{1}{\pi} \quad P \int_{-\infty}^{\infty} \frac{f(\lambda)}{\lambda-\lambda_{0}} d \lambda
$$

Now if we write $V=v^{j \theta}$, then $f(\lambda)=\operatorname{lnV}=\operatorname{lnv} \pm j \theta$, and we can equate imsginary parts in $(A-2)$ and get

$$
\theta\left(\lambda_{0}\right)=+\frac{1}{\pi} P \int_{-\infty}^{\infty} \frac{\ln v(\lambda)}{\lambda-\lambda_{0}} d \lambda
$$

where $V^{2}$ is the reflectivity.

By a simple transformation, we can obtain

$$
\theta\left(\lambda_{0}\right)=\frac{1}{\pi} P \int_{0}^{\infty} \frac{\lambda[\operatorname{lnv}(\lambda)-\operatorname{lnv}(-\lambda)]+\lambda_{0}[\operatorname{lnv}(\lambda)+\operatorname{lnv}(-\lambda)]}{\lambda^{2}-\lambda_{0}^{2}} d \lambda
$$

and, making the formal assumption that $\mathrm{v}(\lambda)=\mathrm{v}(-\lambda)$,

$$
\theta\left(\lambda_{0}\right)=\frac{2 \lambda_{0}}{\pi} P \int_{0}^{\infty} \frac{\operatorname{lnv}(\lambda)}{\lambda^{2}-\lambda_{0}^{2}} d \lambda
$$


and an integration by parts gives us

$$
\theta\left(\lambda_{0}\right)=\frac{1}{\pi} \int_{0}^{\infty} \frac{v^{\prime}(\lambda)}{v(\lambda)} \ln \left|\frac{\lambda+\lambda_{0}}{\lambda-\lambda_{0}}\right| d \lambda,
$$

form which is sometimes more convenient.

To show that our proposed equations for the reflectivity satisfy the above relations regardless of the values of the coefficients, it is sufficlent to show that the $V(\lambda)$ defined by the equations is analytic in the lower half of the $\lambda$ plane, is never zero there, and that for real $\lambda,|V(-\lambda)|=|V(\lambda)|$. The first is easy to show; since the coefficients are all positive, it is easily seen that each term's denominator can be zero only for $\lambda$ 's with a positive imaginary part. The last condition also holds: changing the sign of $\lambda$, when $\lambda$ is real, has the sole effect of changing the sign of the imaginary part of $n^{2}$ and so of $n$; whlch leaves $\left|\frac{n-1}{n+1}\right|$ unaffected.

Table 1. Data Obtained with 3M Velvet yellow paper in First Reflection Cavity

\begin{tabular}{|c|c|c|c|c|c|c|c|c|c|}
\hline \multirow{3}{*}{ Prial } & \multirow{3}{*}{$\begin{array}{l}\text { Viewling } \\
\text { Configurationl/ } \\
\text { Theoretical, centef } \\
\text { point 1llumination }\end{array}$} & \multicolumn{5}{|c|}{ I/R Ratio2/ } & \multirow{2}{*}{\multicolumn{3}{|c|}{ Inearity ${ }^{3 /}$}} \\
\hline & & 0.5 & 0.75 & 1.0 & 1.5 & 2.0 & & & \\
\hline & & 0.800 & 0.640 & 0.500 & 0.308 & 0.200 & 0.751 & 0.500 & 0.253 \\
\hline 101 & $\begin{array}{l}\text { Black sleeve, light } \\
\text { pipe, .030" beam }\end{array}$ & $.832 \pm 1$ & $.648 \pm 2$ & $.509 \pm 2$ & . $311 \pm 1$ & $.191 \pm 1$ & .745 & .493 & .257 \\
\hline 104 & Black sleeve only & $.832+5$ & $.683 \pm 1$ & $.561 \pm 3$ & $.332 \pm 2$ & $.189 \pm 1$ & .749 & .498 & .251 \\
\hline 105 & $\begin{array}{l}\text { White sleeve, opal } \\
\text { glass view cap, } \\
\text { light pipe }\end{array}$ & $.822+4$ & $.563 \pm 0$ & $.426 \pm 0$ & $.272+0$ & $.178+0$ & .747 & .498 & .251 \\
\hline 106 & $\begin{array}{l}\text { Same as 105, but } \\
0.075^{\prime \prime} \text { beam }\end{array}$ & $.828+4$ & $.561 \pm 1$ & $.443 \pm 2$ & $.278 \pm 0$ & $.182 \pm 0$ & .745 & .499 & .249 \\
\hline 107 & $\begin{array}{l}\text { Long black sleeve, } \\
0.075^{\prime \prime} \text { beam }\end{array}$ & $.839 \pm 6$ & $.696 \pm 1$ & $.570 \pm 2$ & $.366 \pm 4$ & $.184 \pm 1$ & - & - & .250 \\
\hline 108 & $\begin{array}{l}\frac{1}{2} " \text { baffle colli- } \\
\text { mator, } 0.075^{\prime \prime} \text { beam }\end{array}$ & .844 & .707 & .592 & .371 & .201 & 一 & - & - \\
\hline 109 & $\begin{array}{l}\frac{1}{4} " \text { baffle colli- } \\
\text { mator, } 0.075^{\prime \prime} \text { beam }\end{array}$ & $.842 \pm 2$ & $.702 \pm 1$ & $.578 \pm 2$ & $.392 \pm 1$ & $.173 \pm 1$ & .746 & .496 & .247 \\
\hline 110 & $\begin{array}{l}\text { Same as 109, EMI } 5685 \\
\text { tube } 0.075^{\prime \prime} \text { beam }\end{array}$ & .841 & .704 & .573 & .368 & .192 & .746 & .496 & .248 \\
\hline 111 & $\begin{array}{l}\text { White sleeve, light } \\
\text { pipe, } 0.075^{\prime \prime} \text { beam }\end{array}$ & $.843 \pm 3$ & $.641 \pm 1$ & $.501 \pm 1$ & $.3 \mu_{4 \pm 1}$ & $.200 \pm 1$ & .745 & .496 & .250 \\
\hline
\end{tabular}

1 See figure 7 .

2f I values indicate total spread in two measurements. Only one measurement was made if no \pm value is given.

3) Sector disc attenuator check.

4 From relation $f=1 /\left[I+(L / R)^{2}\right]$. 
Table 2. Data Obtained with Sha.llow Cavity Lined with 3M Velvet Paper

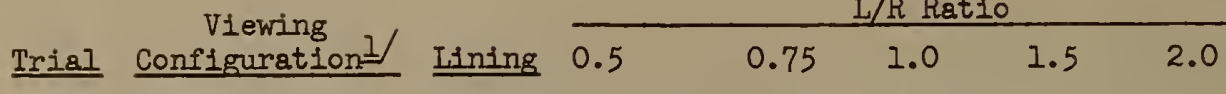

102

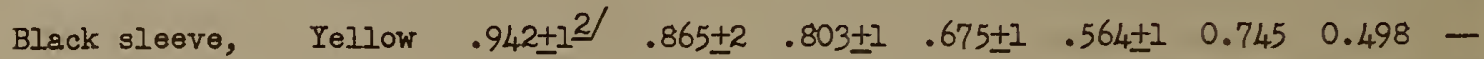
light pipe

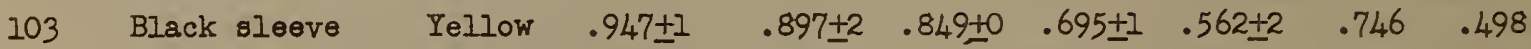
only

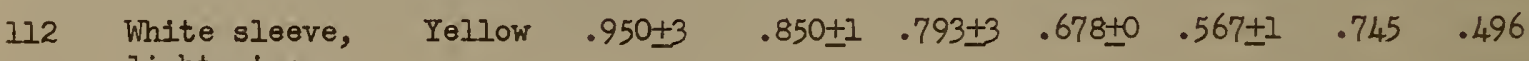
light plpe, $0.075^{\prime \prime}$ beam

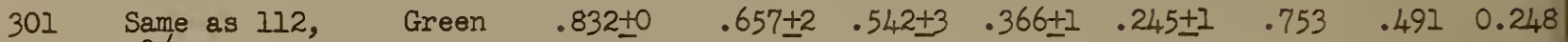
F3

302

Same as 112

Green

$.811 \pm 1 \quad .608 \pm 2 \quad .479 \pm 2 \quad .304 \pm 1 \quad .190 \pm 1$

1) See Table 1 .

2) \pm values indicate total spread in two measurements.

3/ First reflection cavity

4 Sector disc attenuator check.

Table 3. Absolute Reflectance Computed from Shallow Cavity Measurements

A. Method 1 Analysis, $F=f$.

$\underline{\operatorname{Tr} 1 a l}$

102

103

112

302

\begin{tabular}{ll}
\hline 0.5 & 0.75 \\
$.880 \pm 3$ & $.853 \pm 4$ \\
$.893 \pm 3$ & $.897 \pm 3$ \\
$.900 \pm 7$ & $.830 \pm 2$ \\
$.362 \pm 3$ & $.146 \pm 15$
\end{tabular}

$L / R$

1.0

1.5

2.0

$.860 \pm 1$

$.903 \pm 0$

$.850 \pm 3$

$.270 \pm 15$

$.850 \pm 1$

$.863 \pm 1$

$.833 \pm 1$ $.832 \pm 2$

$.853 \pm 0$

$.836 \pm 1$

$.315 \pm 5$

B. Method 2 Analysis, $f=F$.

$L / R$

Trials

$101-102$

$103-104$

$111-112$

$301-302$

$$
0.5
$$

$.851 \pm 3$

$.867 \pm 3$

$.874 \pm 10$

$.274 \pm 4$
0.75

$.849 \pm 3$

$.879+3$

$.830+2$

$.333 \pm 13$
1.0

$.856 \pm 2$

$.884+0$

$.849 \pm 3$

$.355 \pm 13$

1.5
$.848 \pm 1$
$.856 \pm 1$
$.850 \pm 0$
$.329 \pm 5$

1.5

2.0

$.834 \pm i$

$.836 \pm 2$

$.835 \pm 2$ $.305 \pm 7$

C. Method 3 Analysis, F for first reflection, f for all others.

$\mathrm{L} / \mathrm{R}$

Trials

$101-102$

$103-104$

$111-112$

$301-302$
0.5

$.786 \pm 2$

$.802 \pm 2$

$.805 \pm 9$

$.260 \pm 0$
0.75

$.842 \pm 3$

$.837 \pm 3$

$.829 \pm 2$

$.356 \pm 10$
1.0

$.851 \pm 2$

$.845 \pm 0$

$.849 \pm 2$

$.365 \pm 11$

\section{5}

$.847 \pm 1$

$.846 \div 2$

$.847 \pm 0$

$.320+3$
2.0

$.840=1$ $.840 \pm 2$ $.824 \pm 1$ $.307 \pm 3$ 
Table 4. Reflectance of Various Materials at 0.6328 Micron, Rolative to 3M Velvet Yellow Paper

Specimen

Specimon

3M Velvet yellow over white bond (standard)

3M Velvet yellow over brass

3M Velvet green over white bond

3M Velvet white over white bond

3M Velvet black over white bond

$\mathrm{ZrO}_{\mathbf{2}}$ \#I

$\mathrm{ZrO}_{2} \# 2$

$\mathrm{CaZrO}_{3} \# 1$

$\mathrm{CaZrO}_{\mathrm{B}} \# 2$

$\mathrm{ThO}_{2} \# 1$

$\mathrm{ThO}_{2}$ \#2

$A I_{2} \mathrm{O}_{3} \# 1$

$\mathrm{Al}_{2} \mathrm{O}_{3} \# 2$

$\mathrm{ZrO}_{3} \# 1$

$\mathrm{ZrO}_{2} \# 2$

Rhodium Mirror $4 /$

Aluminum Merror

Gold Mirror

Silver Mirror

Porcelain Enamel \#1

$\begin{array}{lll}" & \text { " } & \text { \#2 } \\ \text { " } & \text { " } & \text { \#3 } \\ \text { " } & \text { " } & \# 4 \\ \text { " } & \text { " } & \text { \#5 } \\ \text { " } & \text { " } & \text { \#6 } \\ \text { " } & \text { " } & \text { \#8 }\end{array}$

Relative Reflectance

Laser

Commercial

Reflectometer Reflectometer 2/ Total

1.000

1.000

0.991

$0.373 \pm 13$

$1.025 \pm 6$

$0.044 \pm 1$

$1.040 \pm 2$

$1.039 \pm 2$

$0.987 \pm 2$

$0.953 \pm 3$

$1.065 \pm 5$

$1.033 \pm 2$

$1.040 \pm 1$

$1.039 \pm 2$

$1.063 \pm 1$

$1.067 \pm 1$

$0.979 \pm 1$

$1.119 \pm 1$

$1.166 \pm 4$

$1.208 \pm 5$

$0.941 \pm 0$

$0.081 \pm 1$

$0.395 \pm 2$

$0.092 \pm 1$

$1.075 \pm 3$

$0.187 \pm 1$

$0.838 \pm 1$

$0.287 \pm 1$
0.364

0.009

2.4

1.030

0.035

$1.039 \pm 1$

$1.060 \pm 1$

$0.992 \pm 2$

$0.972+4$

$1.079 \pm 3$

$1.050 \pm 2$

$1.020 \pm 30$

$1.028 \pm 30$

$1.060 \pm 2$

$1.060 \pm 3$

$0.955 \pm 26$

0.005

0.009

0.001

0.021

0.005

0.019

0.014

0.017

0.020

0.011

0.003

0.007

0.024

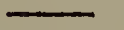

0.915

0.026

2.8

0.064

0.017

35.9

0.373

0.022

5.9

1.072

0.003

0.3

0.167

0.020

10.7

0.825

0.013

1.58

0.263

9.13

I/ Relative to Commercial Reflectometer Value

2/ Data without \pm value is from G.E. Recording Spectrophotometer. The \pm value is total spread of two readings of Cary Model 14 Spectrophotometer.

3/ The \pm value 18 total range of three measurements.

4 Not the same specimen. 
Table 5. Parameters and Relations Descriptive of Wave- Matter Interaction [21]

Complex Permittivity

Complex Permoablitity

Complex Propagation Factor $\gamma=\alpha+j \beta$

Complex Dielectric Constant $K^{*}=\frac{\epsilon^{*}}{\epsilon_{0}}=K^{\prime}-j K^{\prime \prime}$

Complex Index of Refraction $n *=n-n j k$

Intrinsic Impedance

$$
Z^{*}=\sqrt{\frac{\mu^{*}}{\varepsilon^{*}}}
$$

$\gamma=j \omega\left(\epsilon^{*} \mu *\right)^{\frac{1}{2}}$ $\epsilon^{\prime}$ - Real Permittivity

$\epsilon^{\prime \prime}$ - Loss Factor

Valid Assumption for Almost All Applicatlons

$\alpha$ - Attenuation Factor

B - Phase Factor

$K^{\prime}$ - Relative Permittivity or Real Dielectric Constant

$K^{\prime \prime}$ - Relative Loss Factor

n - Index of Refraction, Ratio of Phase Velocities

$\mathrm{k}$ - Index of Absorption

$Z^{*}$ - Ratio of Electric to Magnetic Field Vectors

$\epsilon^{*}=\epsilon_{0}\left(1-\rho \frac{\sigma}{\omega}\right)$

Table 6. Typical Values of Parameters Descriptive of Wave-Matter Interaction [27]

Material,

Temperature

and Wavelength

Theoretical

Conditions

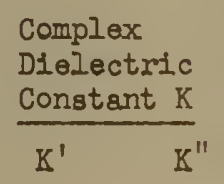

$$
\alpha\left(\mu^{-1}\right) \quad \beta\left(\mu^{-1}\right)
$$

$-6.69 \quad 5.74$

1.03

2.69

3.5

0.91

1.62 .0

1.62

2.72

$-16.8 \quad u_{4} .3$

(2.72

2.81

$4.49 x$

1.675

$8.00 x$

$10^{-6}$
Complex

Refraction $n$

k $10^{-7}$
Intrinsic Impedence Z $z^{\prime}(\Omega) \quad z^{\prime \prime}(\Omega)$

Normel Spectral Entssivity $\varepsilon_{0, \lambda}$

Sapphire

$300 \mathrm{~K}, 4 \mu$

$\begin{aligned} 2.10 \times & 2.63 \\ 10^{-6} & \end{aligned}$

630

$5.03 \times-$ 
Table 7. Classical Models for the Prediction of the Emissivity of Metals

gen-Rubens

D. [19]

chkinass

$05[28]$

[29]

hmidt \&

kert

$35[31]$

visson \&

eks

$24[30]$

$$
\varepsilon_{0, t}=.5736\left(\rho_{m} \mathrm{~T}\right)^{\frac{1}{2}}-.1769\left(\rho_{m} \mathrm{~T}\right)
$$

$\epsilon_{0, t}=.5736\left(\rho_{m} T\right)^{\frac{1}{2}}-.1769\left(\rho_{m} T\right)+.058\left(\rho_{m} T\right)^{3 / 2}$

$$
\varepsilon_{h, t}=.751\left(\rho_{m} \mathrm{~T}\right)+\ldots . . \cdot \rho_{m} \mathrm{~T}<.1
$$

$$
\begin{aligned}
& \epsilon_{\mathrm{h}, \lambda}=.476\left(p_{\mathrm{m}} / \lambda\right)^{\frac{1}{2}}-.148\left(p_{\mathrm{m}} / \lambda\right), 0<p_{\mathrm{m}} / \lambda<.5 \\
& \epsilon_{\mathrm{h}, \lambda}=.442\left(\rho_{\mathrm{m}} / \lambda\right)^{\frac{1}{2}}-.0995\left(p_{\mathrm{m}} / \lambda\right), .5<p_{\mathrm{m}} / \lambda<2.5 \\
& \epsilon_{\mathrm{h}, \mathrm{t}}=.751\left(\rho_{\mathrm{m}} \mathrm{T}\right)^{\frac{1}{2}}-.396\left(\rho_{\mathrm{m}} \mathrm{T}\right), 0<\rho_{\mathrm{m}} \mathrm{T}<.2 \\
& \epsilon_{\mathrm{h}, \mathrm{t}}=.698\left(p_{\mathrm{m}} \mathrm{T}\right)^{\frac{1}{2}}-.266\left(p_{\mathrm{m}} \mathrm{T}\right), .2<\rho \mathrm{T}<.5
\end{aligned}
$$

rker \& bott 64 [32]

where
Also called the DrudeZener relations; applicable at long wavelengths and/or high temperature; derived from classical electromagnetic theory assuming negligible phase change of electrlc field within the metal.

Obtained by Integration of Hagen-Pabens' first term with Planck distribution curre, when combined with $\rho$ vs $T$ linearity relation then radiant energy is proportional to the fifth power of the temperature.

Obtained by integration of Hagen-Rubens' first and second terms with Planck Distribution curve.

Using the Fresnel Relation for angular dependency, hemispherical emissivity is obtained.

With graphical integration techniques, the limit of applicability of the Davisson-Weeks Model is entended.

Assumed the Drude Theory with finite relaxation time; expressions for $\epsilon_{0, t}, \varepsilon_{h, \lambda}$, and $\varepsilon_{h, t}$ are also developed. 
Table 8. Theoretical Models for Prediction of the Optical Properties of Metals

\section{FreQ-Electron}

Assumes free electrons of one type are subjected to simusoidal electric field and damped by a viscous force.

$$
\mathrm{R}^{*}=1-\left(\frac{\lambda}{\lambda_{0}}\right)^{2} \frac{1+j\left(\frac{\lambda}{\lambda_{f}}\right)}{1+\left(\frac{\lambda}{\lambda_{f}}\right)^{2}}
$$

\section{Multi-Free/Bound}

Assumes several types of free and bound electrons are subjected to viscous damping anc elastic restoring forces.

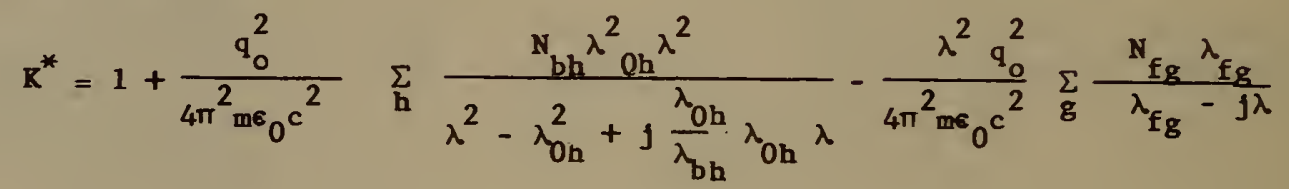

\section{Roberts}

Same form as above, but no significance can be attached to constants. Shown to be valid for all metals and temperatures considered.

$$
\mathrm{R}^{*}=1+\sum_{\mathrm{m}} \frac{\mathrm{K}_{\mathrm{om}} \lambda^{2}}{\lambda^{2}-\lambda_{\mathrm{sm}}+\mathrm{j} \delta_{\mathrm{m} s \mathrm{sm}} \lambda}-\frac{\lambda^{2}}{2 \pi c \varepsilon_{0}} \sum_{\mathrm{n}} \frac{\sigma_{\mathrm{n}}}{\lambda_{\mathrm{rn}}-j \lambda}
$$

\begin{tabular}{|c|c|c|c|}
\hline Electron Type & $\begin{array}{l}\text { Roberts' } \\
\text { Constants } \\
\end{array}$ & $\begin{array}{l}\text { Multi-Free/Bound } \\
\text { Parameters }\end{array}$ & Physical Significance \\
\hline Free & $\sigma_{\mathrm{n}}$ & $\frac{q^{2} N_{f g}}{2 \pi c m \lambda_{f g}}$ & $\begin{array}{l}\text { Contribution to conductivity by free } \\
\text { electrons, type g, when } \lambda \rightarrow \infty \text {, the suman } \\
\text { tion of contributions by all types gives } \\
\text { DC electrical conductivity. }\end{array}$ \\
\hline Free & $\lambda_{\mathrm{rm}}$ & $\lambda_{f g}$ & $\begin{array}{l}\text { Relacation wavelength of free electorns } \\
\text { giving an Indication of time between } \\
\text { electron collisions. }\end{array}$ \\
\hline Bound & $\mathrm{K}_{\mathrm{om}}$ & $\frac{q_{0}^{2} N_{b h} \lambda_{0 h}^{2}}{4 \pi^{2} m \varepsilon_{0} c^{2}}$ & $\begin{array}{l}\text { Proportional to the ratio of Coulomb } \\
\text { repulsive force between bound electrons } \\
\text { and the elsstic restoring force. }\end{array}$ \\
\hline Bound & $\lambda$ sm & $\lambda_{\mathrm{gm}}$ & $\begin{array}{l}\text { Resonance wavelength of bound electrons } \\
\text { without damping forces present. }\end{array}$ \\
\hline Bound & ${ }^{\delta} \mathrm{sm}$ & $\lambda_{\mathrm{Oh}} / \lambda_{\mathrm{bh}}$ & $\begin{array}{l}\text { Ratio of resonsnce wrvelength to relax- } \\
\text { ation wavelength under condition where } \\
\text { damping and restoring forces are respec- } \\
\text { tively absent. }\end{array}$ \\
\hline
\end{tabular}

Table 9. Comparison of the Roberts' Multi-

$$
\text { Free/Bound Electron Model Constants [27] }
$$


চ

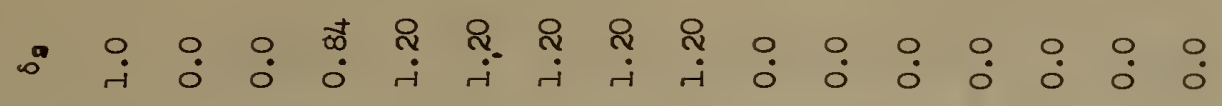

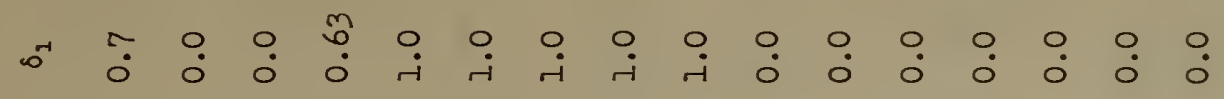

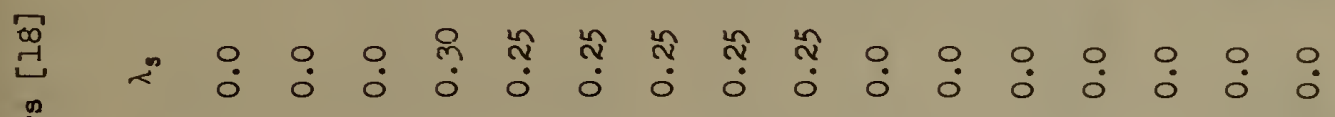

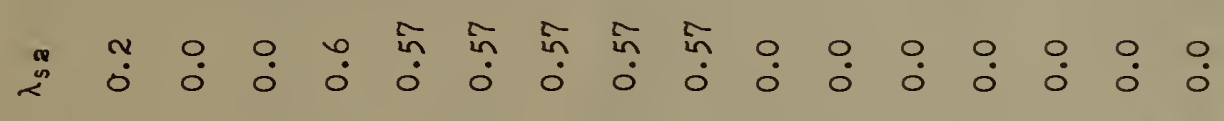

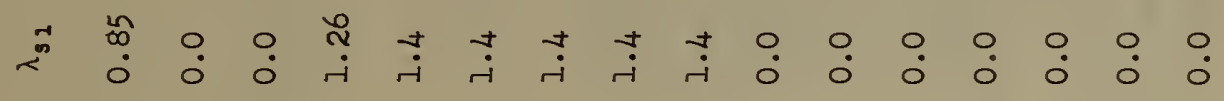

ঋ)

ঋ

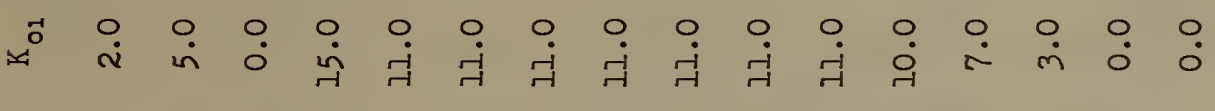

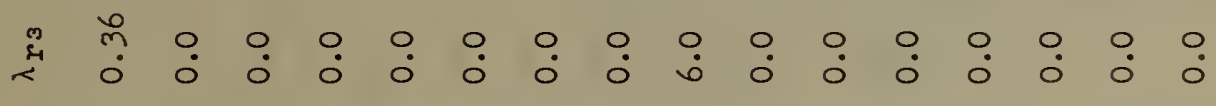

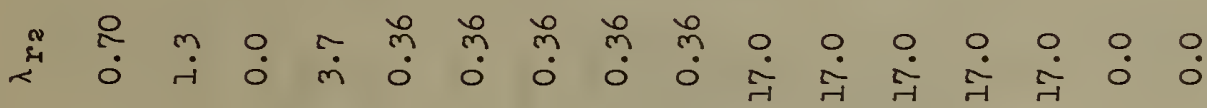

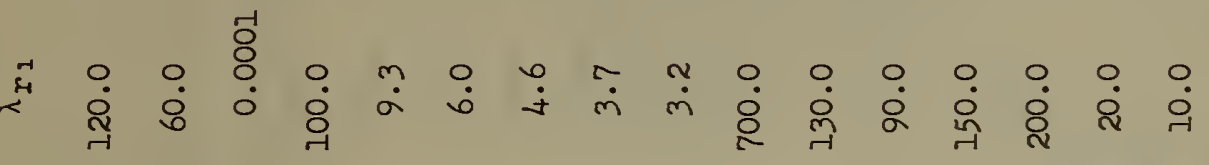

:

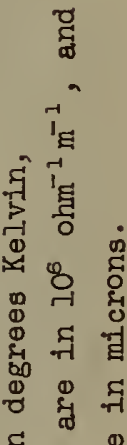

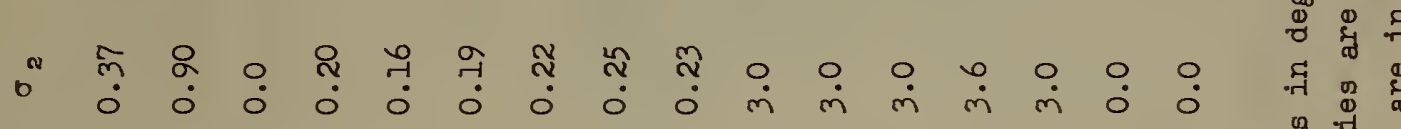

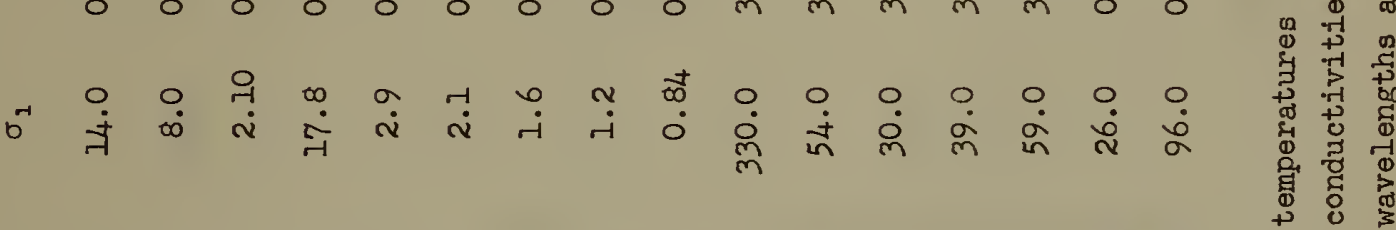

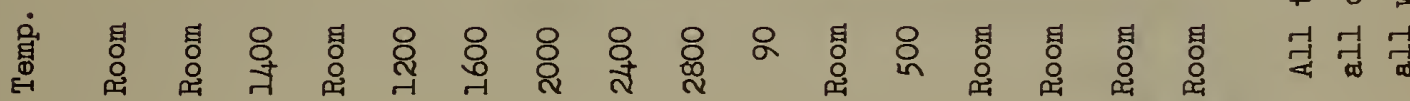

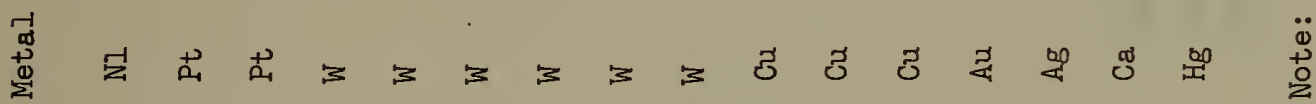




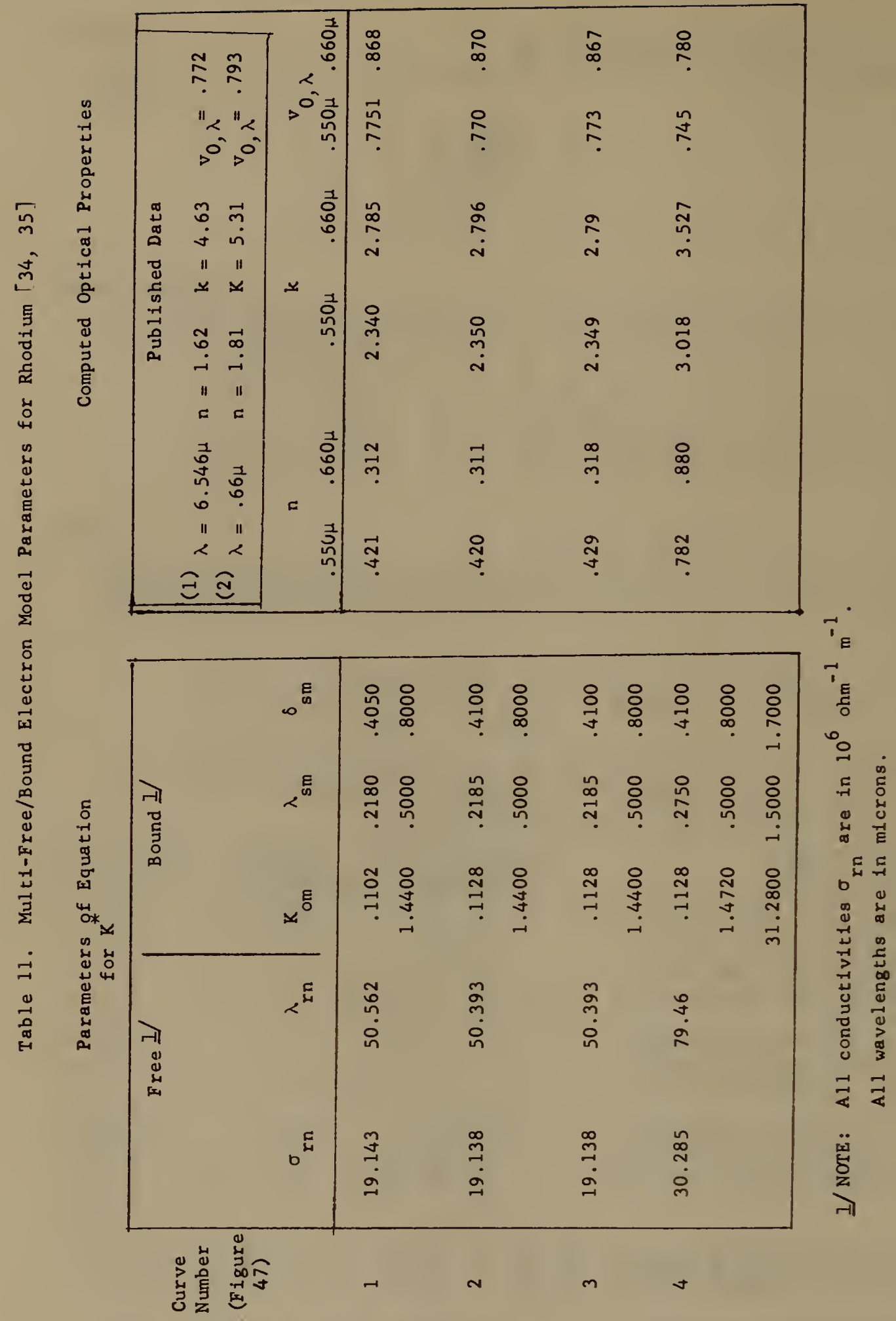


Table 12 Brief Description of Methods of Measurement and Sample Preparation for Cited Literature Values

Material

Platinum

Platinum

Dxidized Inconel

Oxidized Inconel

Platinum

Oxidized Inconel.
Authors

Schatz \& McCandless [41]

Maki, Stair \& Johnson [40]

Maki, Stair \& Johnson [40]

$\mathrm{Blau}$, et al. [42]

Price [39]

Slemp \& Wade [44]
Method/Apparatus

Double-beam recording spectrophotometer with transfer optics to view separate specimen and blackbody furnaces.

Single-beam recording spectrophotometer with transfer optics and traveling optical bench to alternately view blackbody furnace and resistance heated specimen.

Same as above.

Single-beam recording spectrophotometer with specimen imbedded in wall of hollow silicon carbide heating element with cavity to serve as blackbody.

Single-beam recording spectrophotometer with cylindrical specimens electrically heated in vacuum with lateral slit serving as blackbody.

Single-beam recording spectrophotometer views specimen as it rotates past viewing port of blackbody cavity furnace.
Specimen Description

Commercial foil, no additional description given.

Conmercial grade strip ( $\left.8 \frac{1}{2} \times \frac{1}{4} \times \frac{1}{32} 11\right)$ annealed at $1525^{\circ} \mathrm{K}$ for one hour.

Commercial Inconel strip, sandblasted and heated at elevated temperature for six hours.

No details given other than oxidized in air.

Commercial $99.8 \%$ purity sheet.

Oxidized in air for 20 minutes. 
Table 13. Table of Average Standard Deviations

Temperature

Wavelength Range, $\mu$

\begin{tabular}{|c|c|c|c|c|c|}
\hline 12.40 & $27 \cdot 30$ & 26.40 & 32.40 & 32.0 & 37.65 \\
\hline$\underline{\operatorname{Pro}}$ & Bure & Prc & Zure & $\underline{\operatorname{Pr}}$ & dure \\
\hline A & B & A & B & A & B \\
\hline 0.431 & 0.603 & 0.325 & 0.250 & 0.586 & 0.564 \\
\hline .398 & .345 & .326 & .923 & .593 & .447 \\
\hline .402 & .334 & .388 & .504 & .467 & .351 \\
\hline
\end{tabular}

Table 14. Table of Average Measurer nts

Temperature

Wavelength Range, $\mu$

\begin{tabular}{|c|c|c|c|c|c|}
\hline 12.40 & $27 \cdot 30$ & 26.40 & 32.40 & 32.0 & 37.65 \\
\hline$\underline{\operatorname{Pr}}$ & dure & Pro & iure & $\underline{\mathrm{Pr}}$ & aure \\
\hline A & B & A & B & A & B \\
\hline 52.00 & 52.23 & 51.68 & 52.43 & 52.21 & 53.27 \\
\hline 50.53 & 51.78 & 50.52 & 53.21 & 50.66 & 53.44 \\
\hline 50.56 & 50.66 & 50.58 & 51.18 & 50.92 & 51.56 \\
\hline
\end{tabular}



U.S. DEPARTMEMT OF COMHEROE

WASHIRGTOW, D.C. 2020

OFFICINL OUSINESS 